\title{
Application of Monte Carlo to Linac Bunker Shielding Design
}

\author{
Clifford Dugal, B.Eng. \\ September 2006

\begin{abstract}
A thesis submitted to the
Faculty of Graduate Studies and Research

in partial fulfilment of the requirements

for the degree of

Master of Science
\end{abstract} \\ Ottawa-Carleton Institute for Physics \\ Department of Physics, Carleton University \\ Ottawa, Ontario, Canada \\ (C) Clifford Dugal 2006
}




$\begin{array}{ll}\begin{array}{l}\text { Library and } \\ \text { Archives Canada }\end{array} & \begin{array}{l}\text { Bibliothèque et } \\ \text { Archives Canada }\end{array} \\ \begin{array}{l}\text { Published Heritage } \\ \text { Branch }\end{array} & \begin{array}{l}\text { Direction du } \\ \text { Patrimoine de l'édition }\end{array} \\ \begin{array}{l}\text { 395 Wellington Street } \\ \text { Ottawa ON K1A ON4 }\end{array} & \begin{array}{l}\text { 395, rue Wellington } \\ \text { Ottawa ON K1A ON4 } \\ \text { Canada }\end{array}\end{array}$

Your file Votre référence ISBN: 978-0-494-23359-7 Our file Notre référence ISBN: 978-0-494-23359-7

NOTICE:

The author has granted a nonexclusive license allowing Library and Archives Canada to reproduce, publish, archive, preserve, conserve, communicate to the public by telecommunication or on the Internet, loan, distribute and sell theses worldwide, for commercial or noncommercial purposes, in microform, paper, electronic and/or any other formats.

The author retains copyright ownership and moral rights in this thesis. Neither the thesis nor substantial extracts from it may be printed or otherwise reproduced without the author's permission.
AVIS:

L'auteur a accordé une licence non exclusive permettant à la Bibliothèque et Archives Canada de reproduire, publier, archiver, sauvegarder, conserver, transmettre au public par télécommunication ou par l'Internet, prêter, distribuer et vendre des thèses partout dans le monde, à des fins commerciales ou autres, sur support microforme, papier, électronique et/ou autres formats.

L'auteur conserve la propriété du droit d'auteur et des droits moraux qui protège cette thèse. $\mathrm{Ni}$ la thèse ni des extraits substantiels de celle-ci ne doivent être imprimés ou autrement reproduits sans son autorisation.
In compliance with the Canadian

Privacy Act some supporting forms may have been removed from this thesis.

While these forms may be included in the document page count, their removal does not represent any loss of content from the thesis.
Conformément à la loi canadienne sur la protection de la vie privée, quelques formulaires secondaires ont été enlevés de cette thèse.

Bien que ces formulaires aient inclus dans la pagination, il n'y aura aucun contenu manquant.

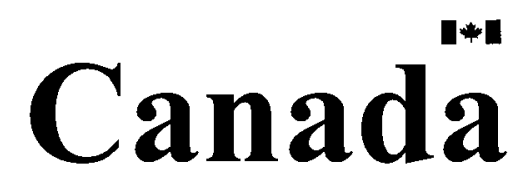




\section{Abstract}

Medical linear accelerators (linacs) are frequently used to treat cancer patients. These linacs are located inside bunkers. The bunker's main purpose is to contain the radiation produced by the linac, such that the people outside the bunker do not suffer from radiation-induced illnesses. The current design method for bunkers relies on simplified equations. These equations do not always result in bunkers being built that match the designed protection criteria. This work aims to look at the use of Monte Carlo for bunker shielding calculations, as Monte Carlo more closely models radiation physics. It is hoped that this better modelling will lead to more accurate bunker designs. In this work, an 18 MV Siemens PRIMUS linac was modelled in MCNP, and promising comparisons to theory, measurements, and calculations were made. 


\section{Acknowledgements}

I, first, want to thank my two thesis supervisors, Dr. David Wilkins and Dr. Miller MacPherson for all their efforts in keeping me on track and helping me to further myself professional and personally. Next, Kenji Myint deserves many thanks for his knowledge of EGS, and for countless insightful discussions. I also would like to acknowledge my various funding sources for keeping me alive over the past few years. Furthermore, Carleton University, and The Ottawa Hospital Regional Cancer Centre are applauded for providing me with exceptional research environments. I am compelled to thank Tim Brecht for his tips on thesis writing ${ }^{1}$.

On a personal note, I need to thank my family and friends. In particular, my parents deserve recognition for always being there and giving me a sense of academic pride, and my very patient and supportive wife, Jen, who acted as content editor.

Finally, I need to thank Uncle Lee's Tea ${ }^{\mathrm{TM}}$. Writing this thesis would have been much more tedious without the countless cups of green tea consumed over the years. 


\section{Table of Contents}

Glossary $\quad$ xii

1 Introduction 1

2 Current Method of Shielding Calculations 5

2.1 Overview of Shielding Calculations by Current Method . . . . . . . . 6

2.2 Problems with Current Method of Shielding Calculations . . . . . . . . . 11

2.3 Review of Related Work . . . . . . . . . . . . . . . . 12

2.3.1 Work Related to General MC Radiotherapy Simulations . . . . . . . 13

2.3.2 Work Related to Linac Photon/Electron Beams . . . . . . . . . 13

2.3.3 Work Related to Neutrons Produced by a Linac . . . . . . . . . . . 16

2.3.4 Work Related to Shielding . . . . . . . . . . . . . . . 19

3 Proposed Method to Improve Shielding Modelling 21

3.1 Use of Monte Carlo for Modelling Entire Bunkers . . . . . . . . . . . . . . 22

3.2 Using MCNP to Model Bunkers . . . . . . . . . . . . . . . . . 24

3.3 Calculating Dose Equivalents in MCNP . . . . . . . . . . . . 26

3.3.1 Neutron Component of Dose Equivalent Calculation . . . . . . . . 28

$\mathrm{V}$ 
3.3.2 Dose Equivalent Calculated using Directly Ionizing Radiation . . . 30

3.3.2.1 Photon/Electron Energy Deposition and Neutron Kerma Method ................... 30

3.3.2.2 Electron Fluence and Neutron Kerma Method . . . . . . 31

3.3.3 Dose Equivalent Calculated using Indirectly Ionizing Radiation . . 32

3.3.3.1 Photon Kerma and Neutron Kerma Method . . . . . . . . 33

3.3.3.2 Photon Fluence and Neutron Fluence Method . . . . . . 34

3.4 Variance Reduction for Monte Carlo Simulations . . . . . . . . . . . 37

4 Modelling of a Linac and Bunker $\quad 40$

4.1 Linac Head Modelling . . . . . . . . . . . . . . . . . . . . . 41

4.2 Radiation Simulation in Linac Head . . . . . . . . . . . . . . 44

4.3 Bunker Modelling . . . . . . . . . . . . . . . . . . . 46

4.4 Radiation Simulation in Bunker . . . . . . . . . . . . . . . . 48

4.5 Calculating a Common Reference for Simulations . . . . . . . . . . . . 51

4.6 Data for Comparison . . . . . . . . . . . . . . . . 53

4.6.1 Measurements in the Maze and Outside the Bunker . . . . . . . . . 53

4.6.2 Head Leakage Measurements . . . . . . . . . . . . . . . . . . . 55

4.6.3 Conventional Method Calculation for Comparisons . . . . . . . . 56

5 Results of Simulations $\quad 58$

5.1 Data Collected from Simulations . . . . . . . . . . . . 58

5.2 Quality of Monte Carlo Calculations ............... 66

5.2.1 Quality of Location 1 Calculations . . . . . . . . . . . . . . . 67

5.2.2 Quality of Location 2 Calculations . . . . . . . . . . . . 67 
5.2.3 Quality of Location 3 Calculations . . . . . . . . . . . . . 67

5.2.4 Quality of Location 4 Calculations . . . . . . . . . . . . . 68

5.2.5 Quality of Location 5 Calculations . . . . . . . . . . . . 68

5.2.6 Quality of Location 6 Calculations . . . . . . . . . . . . 68

5.3 Discussion of Results . . . . . . . . . . . . . . . . . 68

5.4 Comparison to Measurements and Calculations . . . . . . . . . . . 71

5.4.1 Comparison with Published Work . . . . . . . . . . . . 71

5.4.2 Comparisons with Our Measurements and Calculations . . . . . . . 74

6 Conclusions and Future Work $\quad 77$

A Example MCNP Simulation Code for Tallying Dose Equivalent 80

A.1 Dose Equivalent Calculation from *F8:p,e and F6:n Tallies . . . . . . . 81

A.2 Dose Equivalent Calculation from F6:p and F6:n Tallies . . . . . . . . . 83

A.3 Dose Equivalent Calculation from F4:p and F4:n Tallies . . . . . . . . . . . 85

$\begin{array}{lll}\text { B Conventional Shielding Calculations } & 87\end{array}$

B.1 Inside Bunker Door . . . . . . . . . . . . . . . . . . . 87

B.2 Outside Bunker Door . . . . . . . . . . . . . . . . . 91

References 


\section{List of Tables}

4.1 Dose equivalent per MU measured at various locations in the bunker. . . . . 54

4.2 Photon head leakage per MU measured at various locations around the head compared to what was calculated from our model. . . . . . . . . . . . . 55

4.3 Dose equivalent per MU calculated using the conventional approach for the locations immediately inside and outside the bunker door. . . . . . . . . . . 57

5.1 Ambient dose equivalent per MU calculated at various locations in the bunker. 59

5.2 Ratio of Monte Carlo calculated ambient dose equivalent to measured dose equivalent at various locations in the bunker. . . . . . . . . . . . 75

5.3 Ratio of conventionally-calculated dose equivalent to measured dose equivalent for the locations immediately inside and outside the bunker door. . . . 75

5.4 Dose equivalent transmission factors for the bunker door as determined by measurements, conventional approach calculations, and Monte Carlo

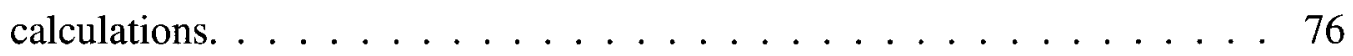

B.1 Photon and neutron dose equivalents per MU calculated for inside the bunker door. . . . . . . . . . . . . . . . . . . . . 91

viii 
B.2 Photon and neutron dose equivalents per MU calculated for outside the bunker door. . . . . . . . . . . . . . . . . . . . 94 


\section{List of Figures}

2.1 Simplified schematic of a conventional bunker design. . . . . . . . . . . 7

4.1 A schematic cross-section of our model of a Siemens PRIMUS linac head. The important parts of the head are labelled. The outer box around the head is the boundary where the phase space was saved. . . . . . . . . . . . . 41

4.2 A horizontal cross-section of the modelled bunker of an $18 \mathrm{MV}$ Siemens PRIMUS linac. The light grey areas are concrete, while the dark grey areas are steel. The numbered black boxes are the locations where ambient dose equivalent was calculated. . . . . . . . . . . . . 47

5.1 Photon fluence at location 1: at the isocentre. . . . . . . . . . 60

5.2 Neutron fluence at location $1:$ at the isocentre. . . . . . . . . . 60

5.3 Photon fluence at location 2 : in the maze. . . . . . . . . . . 61

5.4 Neutron fluence at location 2: in the maze. . . . . . . . . . . . 61

5.5 Photon fluence at location 3: inside the door. . . . . . . . . . . . . 62

5.6 Neutron fluence at location 3 : inside the door. . . . . . . . . . . . 62

5.7 Photon fluence at location 4 : outside the door. . . . . . . . . . 63

5.8 Neutron fluence at location 4 : outside the door. . . . . . . . . . 63 
5.9 Photon fluence at location 5 : in the control area. . . . . . . . . . . . 64

5.10 Neutron fluence at location 5 : in the control area. . . . . . . . . . . . 64

5.11 Photon fluence at location 6: outside bunker, opposite the bunker maze side. 65

5.12 Neutron fluence at location 6: outside bunker, opposite the bunker maze side. 65

5.13 Comparison of the photon fluence spectrum calculated by Sheikh-Bagheri and Rogers ${ }^{27}$ (shown by dashed lines) and the photon fluence spectrum calculated in this work (shown by solid lines) . . . . . . . . . . . 73

5.14 Comparison of the photon fluence spectrum calculated by Sheikh-Bagheri and Rogers ${ }^{27}$ (shown by dashed lines) and the photon fluence spectrum calculated in this work (shown by solid lines). The spectrum calculated by Sheikh-Bagheri and Rogers has been renormalized to have the same area as the spectrum calculated in this work. . . . . . . . . . . 74 


\section{Glossary}

Absorbed Dose: Denoted as $D$, is the quotient of $d \bar{\varepsilon}$ by $d m$, where $d \bar{\varepsilon}$ is the mean energy imparted by ionizing radiation to matter of mass $d m$, thus $D=\frac{d \bar{\varepsilon}}{d m}$. The unit of absorbed dose is joule per kilogram $\left(\mathrm{J} \cdot \mathrm{kg}^{-1}\right)$ and its special name is gray (Gy). ${ }^{2}$

Aligned Field: A hypothetical radiation field in which the radiation flow is unidirectional for the volume of the field. ${ }^{3}$

Ambient Dose Equivalent: Denoted as $H^{*}(d)$, is the dose equivalent that would be produced by the corresponding expanded and aligned field incident on the ICRU sphere, at a depth of $d$ millimetres, on the radius opposing the direction of the aligned field. The recommended value of $d$ for strongly penetrating radiation is $10 \mathrm{~mm}$. The unit of ambient dose equivalent is joule per kilogram $\left(\mathrm{J} \mathrm{kg}^{-1}\right)$ and its special name is sievert (Sv) ${ }^{2,4}$

Auger Electron: An electron emitted from an excited atom as the atom relaxes. ${ }^{5}$

Bremsstrahlung: Photons emitted by electrons being slowed down within the Coulomb field of atomic nuclei. ${ }^{5}$ 
Bunker (Linac): A structure with walls and a ceiling that blocks radiation from exiting, protecting people outside the bunker from harmful radiation exposure from the linac inside.

Cell (MCNP): A three-dimensional geometric volume bounded by first- and second-degree surfaces and/or fourth-degree elliptical tori. ${ }^{6}$

Coherent Scattering: Photon scattering by the combined action of a whole atom. It is an elastic event in that the photon loses essentially none of its energy. ${ }^{5}$

Collision Stopping Power: Denoted as $S_{\mathrm{col}}(E)$, is the component of stopping power related to the energy loss due to collisions with matter. ${ }^{5}$

Compton Scattering: A process under which a photon scatters off an electron, imparting energy to the electron in the process. ${ }^{5}$

Directly lonizing Radiation: Charged particles (e.g., electrons, protons, alpha particles) having sufficient kinetic energy to produce ionization by collision. ${ }^{7}$

Dose Equivalent: Denoted as $H$, is the product of $Q$ and $D$ at a point in tissue, where $D$ is the absorbed dose and $Q$ is the quality factor at that point, thus $H=Q D$. The unit of dose equivalent is joule per kilogram $\left(\mathrm{J} \cdot \mathrm{kg}^{-1}\right)$ and its special name is sievert $(\mathrm{Sv}){ }^{2}$

Expanded Field: A hypothetical radiation field in which the radiation fluence and energy distribution are equal throughout the volume of the expanded field. ${ }^{3}$

Fluence: Denoted as $\Phi$, is the quotient of $d N$ by $d a$, where $d N$ is the number of particles incident on a sphere of cross-sectional area $d a$, thus $\Phi=\frac{d N}{d A} \cdot{ }^{2}$

Gray (Gy): Name for the SI unit of kerma and absorbed dose, equal to $1 \mathrm{~J} \cdot \mathrm{kg}^{-1} \cdot 4$ 
ICRU Sphere: A sphere of $30 \mathrm{~cm}$ diameter made of tissue equivalent material with a density of $1 \mathrm{~g} \cdot \mathrm{cm}^{-3}$ and a mass composition of $76.2 \%$ oxygen, $11.1 \%$ carbon, $10.1 \%$ hydrogen and $2.6 \%$ nitrogen. It is used as a reference phantom in defining dose equivalent quantities. ${ }^{4}$

Indirectly lonizing Radiation: Uncharged particles (e.g., neutrons, photons, gamma rays) that are capable of releasing ionizing charged particles when interacting with matter. ${ }^{7}$

Kerma: Denoted as $K$, is the quotient of $d E_{\mathrm{tr}}$ by $d m$, where $d E_{\mathrm{tr}}$ is the sum of the initial kinetic energies of all the charged ionizing particles liberated by uncharged ionizing particles in a volume element of mass $d m$, thus $K=\frac{d E_{\mathrm{tr}}}{d m}$. The unit of kerma is joule per kilogram $\left(\mathrm{J} \cdot \mathrm{kg}^{-1}\right)$ and its special name is gray $(\mathrm{Gy}){ }^{2}$

Kerma Approximation: An approximation that energy transferred to charged particles by kerma, less the energy that is reradiated by the charged particles, is deposited on the spot, i.e., the collisional kerma is equal to the dose. ${ }^{8}$

Linac: Short for linear accelerator. It is used to accelerate charged particles; in radiotherapy, typically, electrons are accelerated. For the purposes of medical uses, the accelerated particle beam is, normally, expanded and shaped so that affected tissue can be effectively targeted.

Linear Energy Transfer: Also known as linear collision stopping power, $L$, of a material, for a charged particle, is the quotient of $d E$ by $d l$, where $d E$ is the mean energy lost by the particle, owing to collisions with electrons, in traversing a distance $d l$, thus $L=\frac{d E}{d l} \cdot 2$ 
Maze (Bunker): Included in some bunker designs, it is a twisting hall that is designed to stop radiation from directly reaching the entrance way of a bunker.

Monte Carlo (MC) Method: Any method which solves a problem by generating suitable random numbers and observing that fraction of the numbers obeying some property or properties. The method is useful for obtaining numerical solutions to problems which are too complicated to solve analytically. For physical phenomena, the Monte Carlo method is understood as a numerical solution to a problem that models objects interacting with other objects or their environment based upon simple object-object or object-environment relationships. It represents an attempt to model nature through direct simulation of the essential dynamics of the system in question. In this sense the Monte Carlo method is essentially simple in its approach-a solution to a macroscopic system through simulation of its microscopic interactions. ${ }^{9,10}$

Neutron Capture Gamma Ray: A photon released during the process of a neutron being captured by a nucleus. ${ }^{7}$

Pair Production: A process that occurs in the presence of a Coulomb field, under which a photon disappears and gives rise to an electron and a positron pair. ${ }^{5}$

Phantom (Reference): A volume of tissue-equivalent material used to simulate the absorption and scattering characteristics of the patient's body or portion thereof. ${ }^{7}$

Photoelectric Effect: The process under which a photon collides with an atom, ejecting an electron, and the excited atom then emits characteristic photon radiation and/or Auger electrons. ${ }^{5}$ 
Photoneutron: A neutron released from a nucleus as the result of the absorption of an energetic photon. ${ }^{7}$

Quality Factor: A function, $Q$, of unrestricted linear energy transfer, $L$, in water. This function is multiplied with the absorbed dose in a tissue or organ to reflect the relative biological effectiveness of the radiation, the result being the dose equivalent. ${ }^{2,4}$

Radiative Stopping Power: Denoted as $S_{\mathrm{rad}}(E)$, is the component of stopping power related to the energy loss due to radiative interactions. ${ }^{5}$

Relative Biological Effectiveness (RBE): A relative measure of the effectiveness of different radiation types at inducing a specified health effect, expressed as the inverse ratio of the absorbed doses of two different radiation types that would produce the same degree of a defined biological endpoint. ${ }^{4}$

Restricted Stopping Power: Denoted as $L_{\Delta}(E)$, is the part of the collision stopping power that includes all collisions that do not result in energetic electrons with energies more than a cutoff value $\Delta .^{5}$

Sievert (Sv): Name for the SI unit of equivalent dose \& effective dose, equal to $1 \mathrm{~J} \cdot \mathrm{kg}^{-1} .^{4}$

Stopping Power: Denoted as $S(E)$, is the expectation value of the rate of energy loss per unit path length by a charged particle as a function of the charged particle's kinetic energy. It is normally subdivided into two components: collision stopping power and radiative stopping power. ${ }^{5}$

Tenth Value Layer (TVL): The distance of a specified substance which, when introduced into the path of a given beam of radiation, reduces the radiation field quantity to one-tenth of its original value. ${ }^{7}$ 
Tissue Equivalent Material: A material designed to have radiation interaction properties similar to those of soft tissue. ${ }^{4}$

Torus: An (ordinary) torus is a surface having genus one, and therefore possessing a single "hole". The usual torus embedded in three-dimensional space is shaped like a doughnut. $^{11}$ 


\section{Introduction}

Medical linear accelerators (linacs) are frequently employed in medicine to treat cancer patients by focusing a radiation beam on affected tissue. Unfortunately, as we are all aware, radiation is a double-edged sword. When living tissue is exposed to radiation, mutations, caused by an increase in the numbers of free radicals and by direct DNA cleavage, occur. As a result, radiation that can shrink tumours and save lives, can also cause cancer, radiation sickness, cataracts, foetal abnormalities, sterilization, hair loss, skin erythema, and is potentially lethal ${ }^{12}$. Therefore, patients receiving radiotherapy and staff near linacs, need to be shielded from high doses of radiation. These two groups are shielded in very different ways: patients are protected with shielding directly on the linear accelerator; while staff and passerbys are protected by bunkers.

Radiotherapy patients are in an interesting and precarious position where high doses of radiation are need to be focused on an area of tissue containing a malignant tumour and the tissue immediately surrounding this tumour, but should not expose the other areas of the patient's body. This is a serious concern because during the conversion of the narrow electron beam into a larger-area clinically-usable beam, there is a significant amount of radiation that is lost as waste. This waste must be shielded against so that radiotherapy 
patients receive a significant dose of radiation only to the target areas of their bodies. The radiation that gets past the shielding is called leakage radiation.

A typical medical linac accelerates electrons along a linear path. Those electrons are then directed to either a scattering foil or a high atomic number metal. The scattering foil is used to distribute the small electron beam over a larger area; while, the high atomic number metal is used to create a photon beam which is distributed over a large area. These two different beam types have different treatment properties, and the appropriate beam is chosen for radiotherapy ${ }^{13}$. The electron or photon beams are then collimated to the prescribed shape and size by the use of high-density materials. This conversion and shaping of the beam is done in the head of the linac; furthermore, modern linac heads rotate around radiotherapy patients. This beam conversion and shaping together with the head rotation allows for precise targeting of tissue.

To allow for this rotation, the linac shielding needs to be relatively small. As a result, practical linacs will always allow some leakage radiation whenever a radiation beam is being used. Furthermore, practical shielding for linacs cannot block all radiation from reaching people. Indeed, photons from a linac are exponentially attenuated in matter ${ }^{5}$, so it is impossible to completely absorb a photon beam with any finite amount of matter. Linac shielding must be designed to minimize the radiation damage to people, while still remaining functionally and economically practical.

Linacs are located within rooms called bunkers that are designed to prevent the leakage radiation and the radiation that is not completely absorbed by the patient from leaving them. These bunkers separate both the people operating the linacs and the general public in the vicinity from the radiation created by the linacs. Because bunkers need to fit many 
different social and physical constraints, each is designed uniquely. Yet, all bunker designs must sufficiently shield the people in the bunkers' vicinity.

Bunkers are generally designed using massive amounts of concrete and metal, and cost millions of dollars. Obviously, it is in the bunker designer's best interests to design the bunker so that it meets the shielding requirements for that specific location and situation. Although there are design methods that are currently used to quickly calculate bunker shielding quantities (discussed in Chap. 2), these methods use multiple approximations to allow one to calculate shielding quantities with a few analytical equations and, while, the approximations used are generally good, there is almost always a difference between the calculated bunker shielding specifications and the measured specifications. Therefore, it is sometimes necessary that changes be made to the bunker after it is built, and at other times designers and builders purposefully over-shield to compensate for bad approximations. These consequences are very unfortunate as they can be costly and inconvenient.

This work aims to look into improving the method of bunker shielding calculations so that there is a much closer match between designed and measured values. It is hoped that this work could later be refined to be a tool for superior bunker design making bunker shielding more effective and efficient. This work uses a fairly detailed model of a Siemens PRIMUS linac in a local bunker design to gain insight into the expected radiation leakage, and possible improvements to the current design method. The results of this work are discussed in detail in Chap. 4 and Chap. 5.

The method that we are proposing to help calculate bunker shielding uses Monte Carlo (MC), and it is discussed in Chap. 3. MC methods solve a problem by generating suitable random numbers and observing that fraction of the numbers obeying some property or properties. The method is useful for obtaining numerical solutions to problems which 
are too complicated to solve analytically. For physical phenomena, the MC method is understood as a numerical solution to a problem that models objects interacting with other objects or their environment based upon simple object-object or object-environment relationships. It represents an attempt to model nature through direct simulation of the essential dynamics of the system in question. In this sense the MC method is essentially simple in its approach-a solution to a macroscopic system through simulation of its microscopic interactions. ${ }^{9,10}$ The MC method is used to simulate the behaviour of radiation interactions within the bunker at the electron, photon, and neutron interaction levels. Through the accumulation of statistics at the particle level, one can calculate the expected radiation leakage around the bunker.

Using the conventional method, analytic equations can be manipulated to calculate the needed thickness of shielding based on the shielding requirements. The weaknesses of this method are discussed in Sec. 2.2. On the other hand, when one uses Monte Carlo to calculate radiation shielding quantities for a target radiation leakage level, one first must simulate the radiation leakage for a given set of shielding quantities. After examining the results for areas of over- or under-shielding, virtual changes are made to the bunker model. The MC simulation is then re-run. This recursive perturbing of the bunker's shielding and comparison to desired results continues until the calculated radiation leakage is within an appropriate and agreed upon range. It can be easily surmised that each MC simulation takes a very long time to calculate shielding compared to the analytical method. As a result, it is typically more efficient to use an analytical method to calculate an approximate solution, and then to refine the solution using the MC method. 


\section{Current Method of Shielding}

\section{Calculations}

Bunkers are used to shield linac radiation from reaching those people that are not currently being treated. Each bunker is built to match the physical and social requirements of a specific and unique treatment facility. These physical and social requirements include but are not limited to:

- sufficient arresting of radiation from exiting the bunker

- sufficient space for the linac model being used

- sufficient space for equipment (e.g. sink, alignment equipment)

- sufficient space for storage of supplies

- wide enough areas to move people, equipment and supplies in and out of bunker

- appropriate room design (e.g. lighting type, wall type, ceiling type, floor type)

- bunker passages for power, ventilation, data cables

- design that fits within available space 
- design that has enough structural integrity

- cost-effective materials used (material prices change based on markets, locality, etc.)

- reasonable amount of money to build

As these requirements vary between institutions and even between linacs within an institution, bunker designs are highly variable. Verification must be done for each of these designs to assure that they provide sufficient protection for people outside the bunker. In order to construct a bunker that is likely to provide sufficient shielding, methods have been produced that usually result in shielding that at least satisfy the target radiation attenuation. The generally used shielding calculation method is briefly discussed in the following section, followed by a critique of its deficiencies in Sec. 2.2. Finally, a review of work related to what is being explored in this work is presented in Sec. 2.3.

\subsection{Overview of Shielding Calculations by Current Method}

This section's purpose is to familiarize the reader with the current thinking of how bunker shielding is calculated. It is not meant to be a complete introduction to shielding calculations-it is only meant to give the reader enough background to appreciate how one goes about performing the shielding calculations using current methods. With this background in hand, one can contrast the current method with the proposed method discussed in this work. For a more complete description of the current method to calculate bunker shielding, one could review publications from $\mathrm{McGinley}^{14}$ and the NCRP ${ }^{7}$.

There are many different ways to design a bunker, but in general, most bunkers are similar to the conventional bunker design shown in Fig. 2.1. The accelerator is placed in 


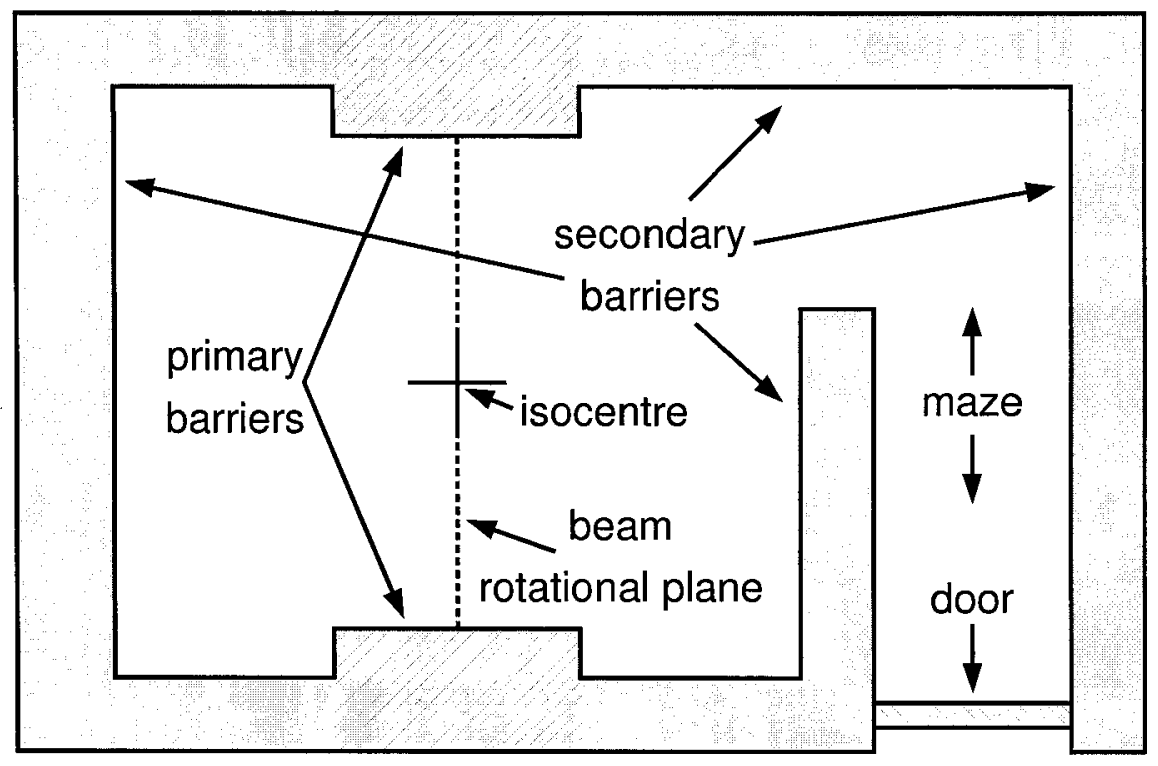

FIG. 2.1. Simplified schematic of a conventional bunker design.

the bunker such that it rotates about the isocentre; which is found in the beam's rotational plane. The accelerator's beam is directed toward the isocentre, where patients are normally positioned. The primary barriers are intended to arrest the accelerator's beam, while the secondary barriers are designed to stop leakage and scattered radiation. Normally, ordinary concrete or heavy concrete (a more dense concrete) is used for the construction of the primary and secondary barriers, although its not uncommon to see the use of lead, steel, polyethylene, earth and wood. The maze is intended to reduce the radiation that reaches the door. The maze accomplishes this by allowing for radiation to be attenuated and absorbed in an increased amount of concrete and air. This reduces the amount of radiation that the door is required to shield; thus, allowing the door to be made lighter compared to a door in a design without a maze. In fact, with some maze designs, the radiation is attenuated so much by the maze that no door is even needed. 
The current method of shielding calculation uses simple equations that are based on approximations of observed macroscopic beam properties. For the normal situation of concrete bunker construction, the primary and secondary shielding thickness are calculated by, first, calculating the target transmission factors for the barriers. These transmission factors are then related to barrier thicknesses via tables and assumed exponential attenuation.

For the sake of the calculations, a shielding design goal of $P$ is set outside the bunker. $P$ is defined as the dose equivalent goal at a point outside the barrier during a week, yielding units of $\frac{\mathrm{Sv}}{\text { week }}$. The NCRP recommends that $P$ is $0.1 \frac{\mathrm{mSv}}{\text { week }}$ for controlled areas and $0.02 \frac{\mathrm{mSv}}{\text { week }}$ for uncontrolled areas. Controlled areas are regions where access is restricted to radiotherapy staff. Uncontrolled areas are regions that are accessible to the general public. In Canada, the Canadian Nuclear Safety Commission (CNSC) does not directly give design targets for bunker shielding. It does, however, give a shielding level for which a full, complicated, assessment of shielding costs and expected doses around the bunker is not mandatory. If this assessment is deemed to not be necessary, much hassle is avoided, and the bunker design is considered to be quite safe. The assessment is deemed to not be mandatory when ${ }^{15}$ :

1. individual occupational doses are unlikely to exceed $1 \frac{\mathrm{mSv}}{\text { year }}$,

2. dose to individual members of the public is unlikely to exceed $50 \frac{\mu \mathrm{Sv}}{\mathrm{year}}$, and

3. the sum of all occupational and public doses over a year is unlikely to exceed $1 \mathrm{~Sv}$.

What follows is a description of shielding for radiotherapy photon beams. Prior to beginning the calculations, the barriers are separated into primary and secondary barriers. For both of these barrier types, a maximal radiation transmission factor that can achieve the 
target shielding design goal is calculated. For primary barriers, the maximal transmission factor for each barrier, $B_{\text {pri }}$, is calculated as:

$$
B_{\mathrm{pri}}=\frac{P d_{\mathrm{pri}}^{2}}{W U T}
$$

where $\quad d_{\mathrm{pri}} \quad$ is the distance in metres from target to protected point

$W \quad$ is the dose in the beam at $1 \mathrm{~m}$ from the target per week (work load)

$U \quad$ is the fraction of time beam falls on the barrier

$T \quad$ is fraction of time a person will be located outside the barrier

For this equation to work, it should be observed that $d_{\text {pri }}$ is referenced to a distance of $1 \mathrm{~m}$ from the source for purposes of the inverse square law, so the right hand side of Eq. (2.1) should be divided by $\left(1 \mathrm{~m}^{2}\right)$.

For the secondary barriers (including primary barriers when the beam is not incident to them), multiple shielding components must be taken into consideration. There is accelerator leakage radiation, scattered radiation from the patient, scattered radiation from walls, and secondary radiations. (e.g., photoneutrons, neutron capture gamma rays). The maximal transmission factor for each barrier's patient scatter radiation component, $B_{\mathrm{ps}}$, is calculated as:

$$
B_{\mathrm{ps}}=\frac{P}{a W T} d_{\mathrm{sca}}^{2} d_{\mathrm{sec}}^{2} \frac{400}{F}
$$


where $a \quad$ is the ratio of scattered radiation at $1 \mathrm{~m}$ from the patient to the primary radiation at $1 \mathrm{~m}$ from target

$$
\begin{array}{ll}
d_{\text {sca }} & \text { is the distance from target to patient in metres } \\
d_{\text {sec }} & \text { is the distance from patient to protected point in metres } \\
F & \text { is the beam area at patient in } \mathrm{cm}^{2}
\end{array}
$$

The factor of 400 is there because $a$ is normalized to a $20 \mathrm{~cm} \times 20 \mathrm{~cm}=400 \mathrm{~cm}^{2}$ field size. The maximal transmission factor for each barrier's leakage radiation component, $B_{\mathrm{L}}$, is calculated as:

$$
B_{\mathrm{L}}=\frac{1000 P d_{\mathrm{sec}}^{2}}{W T}
$$

Scattered radiation from walls and secondary radiation components are generally considered too small to be worthwhile evaluating.

When all the transmission factors are calculated, they are then used to calculate the number of tenth value layers (TVLs) of shielding required to obtain those transmission factors. A TVL is the distance in a specified substance which, when introduced into the path of a given beam of radiation, reduces the radiation field quantity to one-tenth of its original value $^{7}$. The number, $n$, of TVLs of shielding for each of the transmission factors is then:

$$
n=-\log (B)
$$

where $B$ is one of $B_{\mathrm{pri}}, B_{\mathrm{ps}}$ or $B_{\mathrm{L}}$. Unfortunately, TVLs change as a function of radiation energy, so various approximations and assumptions are used to determine an appropriate energy, or energies for thickness calculation. The barrier thicknesses for leakage and scat- 
tered radiation are then combined using a simple method. If the barrier thicknesses differ by more than one TVL, the larger thickness is used; otherwise, if the two thicknesses are approximately equal, the larger of the thicknesses, plus enough extra thickness to reduce the radiation transmission by a factor of two, is used.

After the calculations of the primary and secondary barrier thicknesses are completed, the shielding necessary to protect the areas outside the door must calculated. The door needs to be shielded from radiation including that from the primary beam scattered from room surfaces, scattered head leakage photons, primary scatter from patients, and secondary radiations. As the purpose of this section is simply to give an idea of how shielding is conventionally calculated and because the calculations largely follow the same simplistic form as was discussed for barrier thickness calculations, these equations will not be presented.

\subsection{Problems with Current Method of Shielding}

\section{Calculations}

The conventional bunker shielding design method discussed in the previous section is associated with many concerns. Describing the shielding of bunkers in a dozen or so simple closed-form equations is simultaneously the greatest benefit and the greatest weakness of the conventional method. Simple closed-form calculations makes designing quick, less error-prone, and insightful. Unfortunately, they also oversimplify radiation physics to the point where it's not unusual for radiation measurements to be significantly different from what the equations predicted. In fact, the underlying physical interactions are not directly considered in the calculations-only equations based on macroscopic trends are used. As 
the conventional method is normally conservative in its calculation methods, it is common for bunkers to be over-shielded in areas. This over-shielding costs money-money that could be spent on improving patient care, performing research, or increasing the overall shielding of a bunker.

Another large issue for the conventional method is that it is based on specialized equations that are only valid for rooms that fit certain approximations, causing confusion as to what equation best reflects a design. This can easily lead to poor shielding calculation practises. Additionally, energy spectra and radiation scattering can only be approximated in the conventional method, leading to possibly skewing results because of the poor approximation methods and false assumptions. Finally, inhomogeneities in building material (e.g. steel beams, conduits, ventilation) are not directly handled in the conventional method-they are considered to be a mere perturbation.

\subsection{Review of Related Work}

For those not familiar with the field of MC modelling of external radiotherapy photon beams, Verhaegen and Seuntjens ${ }^{16}$ have produced a topical review. They provide some history in the development of the various MC codes, and compare current codes. They discuss important issues that must be faced when modelling linacs, and, finally, they review conclusions found in previous work. This document is an excellent starting point to familiarize one's self with Monte Carlo as it relates to medical linac modelling. 


\subsubsection{Work Related to General mc Radiotherapy Simulations}

In 1999, Jeraj et al. ${ }^{17}$ utilized both MCNP 4B (Monte Carlo N-Particle transport code ${ }^{18}$, version 4B) and EGS4 (Electron Gamma Shower ${ }^{19}$, version 4) to perform various calculations relevant to radiotherapy. They made comparisons for electron depth dose and backscatter between the two codes, and these codes were compared to actual measurements. It was found that MCNP calculates electron depth dose curves which are too penetrating using the default method energy-indexing algorithm. If the alternate integrated TIGER series-style energy-indexing algorithm is used, the calculated and the measured results agreed much better. It was also found that while EGS under-predicts electron backscattering for high atomic number (high-Z) materials, MCNP handles the situation fairly well. A timing study between the two codes was also done showing that EGS is generally much faster than MCNP. Furthermore, using a large number of scoring volumes dramatically slows down calculations in MCNP, but does not affect EGS to the same extent. However, defining a simulation with MCNP so that it contains a large number of non-scoring geometry volumes does not reduce the simulation speed greatly.

\subsubsection{Work Related to Linac Photon/Electron Beams}

While MC particle transport techniques were first introduced in the 1940s for use in the design of atomic weapons, they did not become popular for use in medical physics until the $1990 \mathrm{~s}^{16}$. Since that time, both EGS and MCNP have been commonly used to model the beam forming components of various models of linacs.

Possibly the most significant tool to be created as of yet for streamlined modelling of the beam-forming components of linacs is $\mathrm{BEAM}^{20}$. It provides a simplified interface for one 
to assemble a variety of elementary geometric entities (jaws, applicators, stacked cones, mirrors, etc.) which the user puts together as needed to model the beam-forming components. It allows the user to select various variance reduction techniques, and allows one to perform in-depth analysis of the radiation interaction within the simulated components.

Chaney et al. ${ }^{21}$ modelled the beam forming components of a $6 \mathrm{MV}$ Siemens MD2 linac in EGS. From this initial research, they determined how each of the head's components scattered radiation.

Years later, Lewis et al. ${ }^{22}$ created a model of the beam forming components of a $4 \mathrm{MV}$ Philips SL 75/5 in MCNP 4B. Using this model, depth dose distributions and dose profiles for various field sizes were calculated, and they compared favourably with measured values.

At the same time, Siebers et al. ${ }^{23}$ used both EGS4 and MCNP 4B to model the beam forming components of a Varian $2100 \mathrm{C}$ at $6 \mathrm{MV}$ and $18 \mathrm{MV}$ beam energies. They used their data to make comparisons between the two simulations' beam properties. While differences were noted, it was concluded that both codes were equally suitable for generating beams for the purposes of dose calculations in patients.

Later, Sheikh-Bagheri et al. ${ }^{24}$ used EGS4 to model the beam forming components of a research linac. Results were compared between the measured and calculated values. Additionally, a small study was done as to how the parameters of the initial electron beam affects the produced photon beam. The parameters studied included the electron beam's energy, energy distribution, radial intensity distribution, divergence, and sweeping angle.

In a very detailed and in-depth study, Lin et al. ${ }^{25}$ used EGS4 to model the beam forming components of a $6 \mathrm{MV}$ Siemens PRIMUS linac. They used this model to calculate the dose distribution in a water phantom. For this setup, they explored the influence of incident electron beam energies, spot sizes, and beam divergence. It was found that the dose 
profile is influenced by energy and spot size, while the depth-dose curve is influenced only by the energy. The beam divergence did not significantly affect the dose profile, so they recommended that the beam be set to be non-divergent. They concluded when tuning the incident electron beam properties to match measured dose, beam energy should be tuned so that the depth-dose curve matches, then the spot size should be tuned so that the dose profile matches. Lin et al.'s work is very interesting in view of this work as a very similar model of linac was used.

In 2002, Sheikh-Bagheri and Rogers ${ }^{26}$ published a paper discussing how they used EGS4 to simulate 9 different photons beams from Elekta, Siemens, and Varian. They studied the incident electron beam parameters and a few mechanical parameters related to the target, primary collimator, and flattening filter. Results were given in terms of effects the parameters have on in-air off-axis factors and central-axis depth-dose distributions. From these results, a method to accurately derive incident electron beam parameters was discussed. When tuning a MC simulation of a linac producing a photon beam, they suggested to first tune the mean energy of the incident electron beam to match depth-dose curves past the depth of maximum dose. The electron beam width is then tuned using in-air off-axis factors. Electron beams should be set to be non-divergent as it is normally not offered by the manufacturers, and there is no practical method to tune this low-effect parameter. Because of the high sensitivity of off-axis factors to the primary collimator's upper opening, and the flattening filter's material and density, it is important to model these components accurately before tuning the incident electron beam's parameters. In a companion paper, SheikhBagheri and Rogers ${ }^{27}$ focused on the results of the 9 different photon beams simulations. In this paper, they calculated the photon energy spectra and average energy distributions and compared them to older published calculations. Next, they separated the spectra into 
primary and scatter components from the primary collimator, the flattening filter and the adjustable collimators. Finally, they calculated the contaminant-electron fluence spectra and the electron contribution to the depth-dose curves.

Lately, Schreiber and Faddegon ${ }^{28}$ used EGS4 to model the beam forming components of a Siemens PRIMUS linac. Electron beams with the energies of 6, 9, 12, 15, 18 and $21 \mathrm{MeV}$ were modelled for the case with the jaws fully open and the applicator removed. The jaw and the applicator are normally used to collimate an electron beam. See Fig. 4.1 for a diagram of a linac head that includes jaws. Variations of thickness, position and lateral alignment of all treatment head components along with variations of electron beam characteristics were studied. Effects of these variations were shown in depth dose distributions and with beam profiles. Just as in Lin et al. ${ }^{25}$, Schreiber et al.'s work is of particular interest to the author of this work because of the model of linac used.

Most recently, Serrano et al. ${ }^{29}$ used MCNPX (MCNP extended) to model the beam forming components of a 25 MV Siemens PRIMUS linac. Using the manufacturer's data for incident electron beam energy, the dose profile and the relative depth-dose distribution were calculated. When compared to measured values, a disagreement was found. It was discovered that the manufacturer's value of $19 \mathrm{MeV}$ for incident electron beam energy was incorrect, and a value of $15 \mathrm{MeV}$ is more reasonable.

\subsubsection{Work Related to Neutrons Produced by a Linac}

Ongaro et al. ${ }^{30}$ used MCNP-GP, a modified MCNP 4B that generates photoneutrons, to calculate the photoneutron spectra for an 18 MV Elekta SL201 linac. These results compared well with measurements from a neutron spectrometer. They continued their work measuring the neutron spectrum using bubble detectors for both a 15 MV Siemens Mevatron and 
an 18 MV Elekta SL201 linac ${ }^{31}$. Again using MCNP-GP, they then modelled the Siemens and Elekta beam forming components, and calculated the neutron spectra. The measured and calculated values were then compared.

Chibani and $\mathrm{Ma}^{32}$ built on Ongaro et al.'s work ${ }^{30,31}$ by using MCNPX to model the beam forming components of an $18 \mathrm{MV}$ Siemens linac, and also a $15 \mathrm{MV}$ and an $18 \mathrm{MV}$ Varian linac. The simulation was rather complete; simulating electrons, photons, and neutrons with enough detail to be able to calculate an accurate estimate of dose components in and around the beam. The dose, in a phantom from neutrons, protons, and alpha particles, was investigated. Field size effects were also studied. It was found that for a $10 \times 10 \mathrm{~cm}^{2}$ field size with an $18 \mathrm{MV}$ Siemens linac at dose maximums, $0.823 \mathrm{mSv}$ dose equivalent from particles other than photons/electrons is deposited for every $1 \mathrm{~Gy}$ of dose of photons/electrons.

Followill et al. ${ }^{33}$ offered a very useful study of the neutron fluence for 36 linacs, including models from Varian, Siemens, Elekta/Philips, and General Electric. The results were obtained by using gold foil activation measured at various points in the patient plane. These measurements are useful for those calculating treatment room door shielding and for those that are verifying their $\mathrm{MC}$ calculations for a certain linac.

Barquero et al. ${ }^{34}$ measured the neutron spectrum around an $18 \mathrm{MV}$ Siemens Mevatron KD-S using thermoluminescence devices (TLDS). Using their method, they produced spectra with 26 energy bins. They also separately measured the neutron and photon dose equivalents at 21 different locations within the bunker. MCNP 4C was used in two different ways for their work: to calculate the neutron kerma contribution to two different TLDs, and to calculate the neutron spectrum at the points where it was also measured. Both neutron spectrum calculations and simulations compared well to one another. The spectra showed three distinct regions: a peak around $0.1 \mathrm{MeV}$, a flat epithermal region and a thermal re- 
gion. Neutrons become thermalized through collisions with nuclei until their kinetic energy is similar to that of room-temperature air. Dose measurements showed patient dose equivalents of $0.5 \mathrm{mSv}$ and $5 \mathrm{mSv}$ from neutrons and photons, respectively, per treatment Gray. At the same time, they also modelled a crude linac head and bunker in MCNPX 2.4.0. In this bunker they placed a relatively detailed model of a female patient. The purpose of their work was to study the absorbed photoneutron dose to patients undergoing radiotherapy in an 18 MV Siemens Mevatron KD-s linac beam; ergo, only a neutron source was considered. Once their simulated neutron spectrum suitably matched a measured neutron spectrum, they calculated the neutron doses to various anatomical regions for a four-field pelvic box treatment.

Pena et al. ${ }^{35}$ further advanced the use of MCNPX 2.4.0, when they used it to model a 15 MV Siemens PRIMUS linac head placed within a bunker. The purpose of this study was to look at photoneutron production and neutron distribution within the room. They simulated the electron, photon, and neutron transport, although photon and electron histories were terminated at $7 \mathrm{MeV}$ since below this value very few photoneutrons are produced, and simulation speed can be improved dramatically. Different modelling approximations were performed so that one could see the effects of simulating more or less accurate geometries on the neutron fluence distribution inside the bunker. It was found that when other massive head components were added to a simulation containing only components to form an electron/photon beam, an $80 \%$ increase in neutron fluence was observed. Finally, neutron fluence maps within the bunker were shown for fast, epithermal, and thermal groupings of neutron energies. 


\subsubsection{Work Related to Shielding}

DeMarco et al. ${ }^{36}$ used MCNP $4 \mathrm{~A}$ to perform thick target bremsstrahlung calculations. Bremsstrahlung from $15 \mathrm{MeV}$ electrons hitting targets of beryllium, aluminium, and lead was considered. The fluence from the targets was calculated as a function of angle. Also, the total fluence and the mean energy were compared to measurements, and to similar work calculated using EGS4.

In a detailed study, Carinou et al. ${ }^{37}$ modelled various bunkers designs with MCNP 4B for the purposes of studying neutron dose in mazes. They modelled the linac head as a simple $10 \mathrm{~cm}$ thick sphere. The only source particles modelled were neutrons, and their energy distribution was created by calculating the expected evaporation spectrum component and the direct neutron production component. They varied the maze geometries and wall composition, and studied how this affected dose at the door entrance. They verified their results by comparing to measurements of two different bunker designs. It was found that the analytical maze calculations matched the simulations well, but only for maze lengths longer than $8.5 \mathrm{~m}$. They also found that using barytes concrete (a heavy concrete which contains a type of barium mineral causing the concrete to be more dense and to have higher Z-components than light concrete) instead of standard concrete for room shielding material reduced neutron dose at the door entrance by about $20 \%$. Moreover, they found that lining the inside bunker walls with wood or borated polyethylene can significantly reduce neutron dose at the door entrance.

Al-Affan ${ }^{38}$ used MCNP 4.2 to model a radiotherapy room for the purposes of estimating the photon dose and spectrum at the maze entrance for $6 \mathrm{MV}$ and $10 \mathrm{MV}$ beams. Using published linac radiation data, dose results were found to agree with measured quantities, but disagreed with the results calculated by the method discussed in NCRP report no. $51^{39}$ 
(which has since been superseded by NCRP report no. $144^{40}$ ). Because results were calculated by MCNP, it was fairly easy to separate the components of the dose at the door. From this information, suggestions were made as to how the NCRP formula should be improved. McGinley et al. $^{41}$ modelled a bunker with a simplified $18 \mathrm{MV}$ linac with MCNP 4B for the purposes of validating a simple empirical method for estimating the photon dose at the maze door ${ }^{42}$. So that comparisons could be done, measurements of the quantities were performed. It was found that the simulation, the equation, and the measurements all agreed within $26 \%$ for the neutron capture gamma ray dose. A conclusion was made that the predominant photon dose for mazes greater than $3 \mathrm{~m}$ long was from captured gamma rays.

Lin et al. ${ }^{43}$ measured the photoneutron dose equivalents throughout the bunker of a Siemens PRIMUS linac. They used BD-PND bubble detectors and powdered $\mathrm{P}_{2} \mathrm{O}_{5}$ to perform these measurements. For $15 \mathrm{MV}$ photon beams and 15, 18, and $21 \mathrm{MeV}$ electron beams, neutron dose equivalents where measured for a few points near the isocentre. Furthermore, for the $15 \mathrm{MV}$ photon beams, a detailed map of the neutron dose equivalent throughout the bunker was measured.

Most recently, Avila-Rodriguez et al. ${ }^{44}$ used MCNP 4C3 to investigate the backscattering and transmission of high-energy photons in thick slabs of concrete, iron and lead. A broad beam of typical bremsstrahlung photons from a 24 MV linac was used in this study. Using a weight-window variance reduction technique, transmission was studied for penetrations depths of 15 mean-free paths. Calculations agreed well with values recommended by the NCRP and the IAEA. 


\section{Proposed Method to Improve}

\section{Shielding Modelling}

The current analytical method of shielding calculation, while quite straightforward, has the disadvantage of not being able to fully account for the shielding properties of bunkers. This, inevitably, leads to over- or under-shielding. For a more thorough discussion of the problem with the current method of shielding calculation refer to Sec. 2.2. The most accurate method of determining shielding specifications would be to actually build the shield design in question, to perform radiation measurements on it, then change the shield based on the measurements and rebuild the shield until the design is able to meet specifications. Unfortunately, this method would be massively time-consuming and extremely costly. Furthermore, this method would be considerably more dangerous than the paper and pencil methods used with the current analytical calculations. As building test bunkers is impractical, the next best thing would be to fully simulate all of the radiation interaction within bunkers, but again, this is too impractical considering the computational time and resources that would be necessary. The third best option is to partially simulate radiation interactions and problem geometry in such a way that the problem is easier to compute, but the results 
do not differ for the targeted level of accuracy. This is what is done in Monte Carlo (MC) radiation simulations ${ }^{10,6}$.

As computers are now powerful enough to perform meaningful bunker shielding MC radiation simulations, we have chosen to evaluate this method as a future tool to help design bunker shielding. There are many different software packages that have been created to perform MC radiation simulations, each with their own strengths and weaknesses. When choosing a suitable MC software package for this project, only MC packages that are applicable to radiotherapy photon beams were considered for use for our bunker shielding

problem. For these types of MC packages, comparisons have been made between them ${ }^{16}$ so that one can choose appropriate code for their work. Of the publicly available MC codes, we chose MCNP $5^{45,6}$ (Monte Carlo N-Particle transport code, version 5) because it models photonuclear interactions, has powerful geometric modelling interface, and is well-used in the radiotherapy field.

\subsection{Use of Monte Carlo for Modelling Entire Bunkers}

Monte Carlo can be used to model almost anything to do with radiation shielding. If one wanted, it could be used to model a huge concrete slab or an individual piece of dust floating within a room. It can be used to model barriers, ventilation, conduits, and large objects, and look at the radiation patterns in them and around them. The major limitation for Monte Carlo for radiation transport is that for any object being simulated, the important properties (in terms of radiation transport) must be specified. These properties are dimensions, shape, density, and isotopic composition. From these properties, MCNP can determine appropriate interaction cross sections and sample physical interactions accordingly. 
For some items, it is relatively easy to determine dimensions, shape, density, and isotopic composition; for others it is not. For example, an iron block would be fairly simple to define-the dimensions can easily be measured, the shape is simple, and the density and isotopic composition can be obtained from a good atomic data reference. On the other hand, a shoe, is extremely difficult to accurately define-the dimensions and shape are complex, and it is made out of multiple materials that don't have a commonly known density or isotopic composition. So, while it is possible to model everything in and around a bunker, it would be a very tedious task to do.

Another consideration is that the contents of a bunker change on a daily basis, from the placement of a pencil, to the movement of equipment. So, it would be rather pointless to model a bunker perfectly, when it is known that what is being modelled is a moving target.

So, what must be done, is that the bunker must be modelled accurately enough so that the shielding can be evaluated. But, objects should not be specified overly precise such that everyday changes that happen within the bunker are more significant. Exactly how accurately objects should be specified for best results is currently not well explored. One can obtain insight on what is important to model accurately from what the current shielding method takes into consideration. From that, emphasis is put on:

- large, bulk shielding, e.g., walls, ceiling

- door construction

- shielding between door and bunker

- passages through walls for ventilation, power, etc. 


\subsection{Using MCNP to Model Bunkers}

The following is an overview of how MCNP can be used to model bunkers. Information in this section can be studied in more detail using the MCNP 5 manual $^{6}$.

MCNP is a program that performs MC radiation transport simulations. The internal parts of the MC code are not exposed to the user-only a set of parameters to control how the MC simulation is performed are available. This allows the user control of the MC process, but does not let/require the user to change any of the underlying MC processes. This usually allows for sufficient control of what is being modelled, and of how the MC simulation is being performed. Although, as with any large system, there is a trade-off between flexibility and efficiency. Ergo, there is a framework that is followed while using MCNP.

To make use of MCNP, one first defines the isotopic compositions of materials, then one defines the geometry of the problem. Next, one defines the source of the radiation. Following that, one defines what information will be collected from simulations. Finally, one defines the MC parameters of the simulation.

In simulations, each defined volume of study can be of only one homogeneous material. As a result, for every object of a different composition, a new material must be defined. Materials are defined as the sum of the fractional isotopic parts. Multiple data files of radiation interaction tables for different isotopes, called interaction libraries, are provided within MCNP and others are available. Notably, there is a set of photonuclear libraries available from the IAEA ${ }^{46}$ that greatly extends the quality and quantity of photonuclear interactions that MCNP can handle. When defining materials, one chooses an appropriate library from which to obtain interaction data. This choice is made based on the amount of 
data, the temperature at which the data are optimal, the group supplying the data, and the year that the data were collected.

The geometry of the problem is defined in two phases. In the first phase, one defines first- and second-degree surfaces and fourth-degree elliptical tori. In the second phase, one defines how these surfaces should be assembled together to create the desired geometry. For example, to define a cube, six planes are first defined (one for each surface of the cube), next, the volume of the intersection of the six planes is defined to be the cube. MCNP calls this defined volume a cell. Each cell is, also, assigned a material and a density.

MCNP is quite flexible in terms of how a radiation source is defined. It can be defined as a general source of neutrons, photons, or electrons, with an arbitrary distribution of location, direction, and energy. For radiotherapy, this can be useful for defining an incident electron beam, looking at transmission/reflection of arbitrary radiation beams, providing an estimated neutron head leakage spectrum for shielding purposes, examining the dose deposited in tissue by a neutron beam, and many other applications. It is also possible to store the radiation pattern of a simulation's surface to a file called a phase space file. A phase space file contains a listing of all the particles that crossed a given surface, along with each of their positions, direction, and energy.

Phase space files also can be used as a radiation source in a later simulation. This is useful when a series of simulations is being computed, in which the geometry around the source is fixed for the set of simulations, but the geometry away from the source is variable. In this instance, one could break the simulation into two parts-the fixed geometry and the variable geometry. One could, then, record the phase space around the first fixed-geometry simulation, and then use that one phase space as the radiation source in the following varying-geometry simulations. This can save a significant amount of simulation time as 
the fixed-geometry only needs to have radiation transported through it once; allowing this information to be reused. If one still does not have enough flexibility with the previous two methods, provisions have been made so that the user can create their own Fortran 90 source code to produce source particles. It is also possible to use isotopes undergoing fission as a particle source, although that is not of interest in this type of work.

It is possible to score information from simulations in what MCNP calls tallies. Tallies are used to store all the statistical information that is used to calculate and evaluate simulation results. These tallies can be used to determine current, fluence, energy deposition, pulse distribution, and other quantities based on those calculations. One can specify where in the simulation these quantities should be calculated, along with different modifiers for how this scoring should be done. For shielding purposes, we are primarily interested in dose equivalents, and the use of tallies to calculate these quantities is discussed in Sec. 3.3.

There are various other parameters that can be adjusted to tune a simulation. Some of the most important for drastic speed improvements are variance reduction techniques, as is discussed in Sec. 3.4. One also needs to specify criteria for when to end a simulation, whether it be number of particles, or minutes of simulation time. Other parameters that can be specified include tuning how the physics is modelled, and what information is output.

\subsection{Calculating Dose Equivalents in MCNP}

Dose equivalent is simply dose multiplied by an appropriate quality factor to account for the relative biological effectiveness of radiation. Dose is energy deposited by charged particles per unit mass. The quality factor accounts for the extra damage (in terms of biological effect) that more densely ionizing radiation causes. For electrons, the quality 
factor is defined to be unity; therefore, a quality factor expresses how many times more damaging a type of radiation is compared to electrons for the same amount of deposited dose. So, to be rigorous with Monte Carlo, one needs to have all uncharged particles create charged particles, and those charged particles must be followed to determine where dose is deposited.

As charged particles generally interact more strongly with matter and are stopped more quickly than uncharged particles of similar energy, an approximation that local dose is equal to local kerma can be used. That is to say, it is possible to approximate the energy that would be locally deposited by charged particles as the energy locally transferred to the charged particles. So, if there were only photons and electrons within a simulation, there would be two ways to calculate dose: either to measure the dose delivered from electrons, or to ignore the dose from electrons and set the dose to be equal to the kerma from photons. For the latter method, if one somehow accounts for photons created by electrons, one does not actually need to follow electrons at all saving significant simulation time. But, using this approximation is not always appropriate, especially in areas close to a change in material where the energy being deposited by charged particles is not nearly equal to the energy photons are imparting to the charged particles.

To calculate dose in MCNP in a simulation with neutrons, photons, and electrons, the dose is broken into two parts: dose approximated from neutron kerma, and dose approximated from photon kerma \& electrons. For neutron kerma, energy is transferred to many different charged particles types, including protons, alpha particles, and ions, all of which are not handled explicitly by MCNP. These charged particles are stopped quite quickly in matter for medical linacs, so, in most cases, the approximation that local neutron dose is equal to local neutron kerma can used. Fortunately, MCNP can calculate neutron kerma, so this 
approximation is what is used whenever neutron dose is calculated. For photon kerma, energy is transferred only to electrons-particles that MCNP can transport. One then has the choice of either calculating the dose by dose delivered by electrons, or by using the approximation that kerma is equal to dose.

As the dose equivalent from the neutron component can only be calculated in one way in MCNP, this method is presented first in Sec. 3.3.1. Two different methods of calculating the dose equivalent from photons/electrons at the electron dose level are presented in Sec. 3.3.2. A method to calculate dose equivalent from photons/electrons from photon kerma, and a related calculation, the ambient dose equivalent, are presented in Sec. 3.3.3. Examples of these methods in MCNP code are shown in Appendix A. Note that the equations presented in the following sections are not exactly as calculated by MCNP; instead, they are written in a more clear and concise form that is near functionally equivalent to what is actually performed by MCNP. For a full description of how the calculations are done in MCNP, the reader should reference the MCNP manual ${ }^{6}$.

\subsubsection{Neutron Component of Dose Equivalent Calculation}

To calculate the neutron component of dose equivalent, the neutron kerma tally is used. The neutron kerma tally calculates a modified neutron kerma differential in energy averaged over the volume of a cell, $\left(\frac{d \bar{K}_{\mathrm{n}}^{\prime}}{d E_{\mathrm{n}}}\right)$

where $E_{\mathrm{n}} \quad$ is the neutron energy

$$
\begin{aligned}
& d \bar{K}_{\mathrm{n}}^{\prime} \quad \text { is the modified neutron kerma averaged over the volume of } \\
& \text { a cell in the interval between } E_{\mathrm{n}} \text { and } E_{\mathrm{n}}+d E_{\mathrm{n}}
\end{aligned}
$$

This tally is modified from using a pure neutron kerma tally in that provisions are made so 
that kerma cannot be double-counted. This is a necessary change if one wants to approximate dose by kerma.

Double-counting from a pure kerma tally could occur in the following illustrative situation. An uncharged particle produces a charged particle, and the charged particle's energy is assumed to be locally deposited. The charged particle, not depositing all its energy, then creates an uncharged particle. This uncharged particle then produces a charged particle, and the charged particle's energy is again assumed to be locally deposited. As a result, the kerma would be counted twice. In tissue, if this double-counting was not accounted for, dose overestimates of $1 \%, 3 \%$, and $6 \%$ are observed for photon-induced electrons of energies of $3 \mathrm{MeV}, 8 \mathrm{MeV}$, and $15 \mathrm{MeV}$, respectively (as is obtained from the radiation yield calculated by NIST ESTAR ${ }^{47}$ ).

The provision used by MCNP to not double-count kerma is that kerma from an uncharged particle is only tallied if the uncharged particle has no parent particle that was counted as kerma. If this provision was not employed, the double-counting of kerma would cause dose to be overestimated when using the approximation that dose is equal to kerma. The modified neutron kerma is assumed to be completely deposited locally. This kerma approximation is usually quite good since protons are the most important neutron kerma component, and the protons' range in water is about $1.2 \mathrm{~mm}$ for $10 \mathrm{MeV}$ neutrons ${ }^{2}$.

Using the kerma approximation and employing the neutron kerma quality factor as a function of energy, $Q_{\mathrm{n}}\left(E_{\mathrm{n}}\right)$, the neutron dose equivalent component, $H_{\mathrm{n}}$, is calculated by:

$$
H_{\mathrm{n}} \doteq \int_{0}^{\infty}\left(\frac{d \bar{K}_{\mathrm{n}}^{\prime}}{d E_{\mathrm{n}}}\right) Q_{\mathrm{n}}\left(E_{\mathrm{n}}\right) d E_{\mathrm{n}}
$$


The neutron kerma quality factor, $Q_{\mathrm{n}}\left(E_{\mathrm{n}}\right)$, is used to account for the higher relative biological effectiveness of neutron kerma over the relative biological effectiveness of dose from electrons.

\subsubsection{Dose Equivalent Calculated using Directly lonizing Radiation}

For this class of dose equivalent calculation, a simulation that transports neutrons, photons and electrons is necessary. This allows for a fairly exact calculation of dose equivalent, but it is slower than the method of estimating dose equivalent using only indirectly ionizing radiation.

There are two methods to calculate dose equivalent in MCNP for this class of dose equivalent calculation. One method is to use a photon/electron energy deposition tally (see Sec. 3.3.2.1), and the other is to use an electron fluence tally (see Sec. 3.3.2.2). Both of these tallies do not account for energy deposited by neutrons kerma, so this dose component needs to be included separately by using a neutron kerma tally as discussed in Sec. 3.3.1.

\subsubsection{Photon/Electron Energy Deposition and Neutron Kerma Method}

The photon/electron energy deposition tally calculates energy deposited in a cell from photons and electrons, denoted as $E_{\mathrm{D}}$. MCNP does this by calculating the difference of the electron/photon energy transported into the cell and the electron/photon energy transported out of the cell. When the cell being tallied is composed of tissue equivalent material, this tally can be converted to dose equivalent, $H$, by:

$$
H \doteq \frac{E_{\mathrm{D}}}{m}+\int_{0}^{\infty}\left(\frac{d \bar{K}_{\mathrm{n}}^{\prime}}{d E_{\mathrm{n}}}\right) Q_{\mathrm{n}}\left(E_{\mathrm{n}}\right) d E_{\mathrm{n}}
$$


where $m$ is the mass of the cell being tallied.

While this is a very direct method to calculate dose, it is usually significantly slower than the other methods since it takes a very long time for the average deposited photon and electron energy to converge. Furthermore, for simulations that use neutrons, the MCNP manual ${ }^{6}$ warns the user, "Caution: The [energy deposition] tally does not work well with neutrons because of the non-analog nature of neutron transport that departs from microscopic realism at every turn." This means that neutron interactions are only modelled in a statistically-correct way, and that the energy deposition tally does not do well when, e.g., energy is not conserved in neutron interactions. Therefore, it is usually best not to use this tally when neutrons are present if another tally will work.

\subsubsection{Electron Fluence and Neutron Kerma Method}

The electron fluence tally calculates the electron fluence differential in energy averaged

over the volume of a cell, $\left(\frac{d \bar{\Phi}_{\mathrm{e}}}{d E_{\mathrm{e}}}\right)$. Schaart et al. ${ }^{48}$ discuss how to convert this quantity into dose. If the cell being tallied is composed of tissue equivalent material, this tally can be converted to dose equivalent, $H$, by:

$$
H \doteq \frac{V}{m} \int_{0}^{\infty}\left(\frac{d \bar{\Phi}_{\mathrm{e}}}{d E_{\mathrm{e}}}\right) L_{\Delta}\left(E_{\mathrm{e}}\right) d E_{\mathrm{e}}+H_{\Delta, \mathrm{e}}+\int_{0}^{\infty}\left(\frac{d \bar{K}_{\mathrm{n}}^{\prime}}{d E_{\mathrm{n}}}\right) Q_{\mathrm{n}}\left(E_{\mathrm{n}}\right) d E_{\mathrm{n}}
$$

where $V \quad$ is the volume of the cell being tallied

$L_{\Delta}\left(E_{\mathrm{e}}\right) \quad$ is the restricted stopping power as a function of electron energy

$H_{\Delta, \mathrm{e}} \quad$ is the dose equivalent deposited by electron track ends 
The product of $\left(\frac{d \bar{\Phi}_{\mathrm{e}}}{d E_{\mathrm{e}}}\right)$ and $V$ gives the electron track length differential in energy, $\left(\frac{d T_{1}}{d E_{\mathrm{e}}}\right)$. It follows that $\left(\frac{d \bar{\Phi}_{\mathrm{e}}}{d E_{\mathrm{e}}}\right)$ egral of the product of $\left(\frac{d T_{\mathrm{l}}}{d E_{\mathrm{e}}}\right)$ and $L_{\Delta}\left(E_{\mathrm{e}}\right)$ is the total of the energy below $\Delta$ deposited by electrons. If $\Delta$ is set to the minimum energy that MCNP is set to consider electrons for propagation, then it follows that the first term of Eq. (3.3) is the dose equivalent deposited from electrons being propagated. $H_{\Delta, \mathrm{e}}$ is calculated as the dose equivalent carried by electrons that were being propagated, but lost enough energy to cross the energy cutoff. This quantity needs to be estimated ${ }^{48}$ as it is not directly available in MCNP.

This dose equivalent calculation could be more reliable than the one of Sec. 3.3.2.1 because MCNP uses particle track lengths in a cell as a basis of calculation instead of calculating energy differences in and out of a cell. Unfortunately, it is significantly harder to correctly calculate. It also does not consider the dose due to electrons from Compton scattering and the photoelectric effect where the electron set in motion has an energy less than the cutoff. Furthermore, Auger electrons below the energy cut-off could be excluded, too. For these reasons, this dose equivalent calculation method was not considered further.

\subsubsection{Dose Equivalent Calculated using Indirectly lonizing Radiation}

For this class of dose equivalent calculation, the approximation that dose is equal to kerma is used. Ergo, we can neglect the full transport of charged particles and only transport neutrons and photons. The time-consuming complete transportation of electrons can be turned off, usually saving considerable simulation time. MCNP provides the option that upon an event that would normally produce an electron, the electron is still created, but only simplified bremsstrahlung production is considered. In this way, the uncharged particle 
fluence is kept very close to what it would be if full electron transport were to have been used.

The drawback to using this method is that the approximation that dose is equal to kerma is not always appropriate for all calculations, especially close to boundaries between differing media. Therefore, in using this method one must be fairly confident that for the region of interest, and for the accuracy sought, dose does indeed closely match kerma.

For this class of dose equivalent calculation, dose is calculated either from photon and neutron kerma (see Sec. 3.3.3.1), or by photon and neutron fluence using conversion coefficients (see Sec. 3.3.3.2).

\subsubsection{Photon Kerma and Neutron Kerma Method}

Similar to the neutron kerma tally described in Sec. 3.3.1, the photon kerma tally, calculates the modified photon kerma differential in energy averaged over the volume of a cell, $\left(\frac{d \bar{K}_{\gamma}^{\prime}}{d E_{\gamma}}\right)$. If the cell being tallied is composed of tissue equivalent material, this tally can be converted to dose equivalent, $H$, by:

$$
H \doteq \int_{0}^{\infty}\left(\frac{d \bar{K}_{\gamma}^{\prime}}{d E_{\gamma}}\right) d E_{\gamma}+\frac{E_{\mathrm{kill} . \gamma}}{m}+\int_{0}^{\infty}\left(\frac{d \bar{K}_{\mathrm{n}}^{\prime}}{d E_{\mathrm{n}}}\right) Q_{\mathrm{n}}\left(E_{\mathrm{n}}\right) d E_{\mathrm{n}}
$$

where $E_{\mathrm{kill}, \gamma}$ is the total energy of photons killed within the cell.

The $E_{\mathrm{kill}, \gamma}$ term needs to be considered in this calculation since it is normal to kill the transport of particles at a finite energy, as is discussed in Sec. 3.4. The truncation of particle paths at a certain energy results in those track ends being excluded from dose calculations, underestimating the dose if the track end energy is not accounted for (although, in practise, 
the dose underestimation is usually negligible). This term needs to be estimated as it is not directly available in MCNP.

\subsubsection{Photon Fluence and Neutron Fluence Method}

Similar to the electron fluence tally, the photon fluence tally calculates the photon fluence differential in energy averaged over the volume of a cell, $\left(\frac{d \bar{\Phi}_{\gamma}}{d E_{\gamma}}\right)$, while the neutron fluence tally calculates the neutron fluence differential in energy averaged over the volume of a cell, $\left(\frac{d \Phi_{n}}{d E_{\mathrm{n}}}\right)$. These fluences can be converted to dose equivalent with appropriate conversion coefficients. Since the conversion coefficients are free to be chosen, it is possible to chose coefficients that calculate different types of dose equivalents. Of considerable interest to this work are conversion coefficients to calculate ambient dose equivalent at a depth of $10 \mathrm{~mm}^{2,49,50}, H^{*}(10)$.

To be able to understand ambient dose equivalent, two concepts need to be introduced. First is the ICRU sphere ${ }^{51}$ - a $30 \mathrm{~cm}$ diameter tissue-equivalent sphere with a density of $1 \mathrm{~g} \cdot \mathrm{cm}^{-3}$ and a mass composition of $76.2 \%$ oxygen, $11.1 \%$ carbon, $10.1 \%$ hydrogen and $2.6 \%$ nitrogen. Next, is an expanded and aligned field ${ }^{51}$, which is a derived radiation field that is calculated from a reference point in a real radiation field. For an expanded and aligned field, the fluence at the reference point is first made to be unidirectional by rotating each of the fluence components such that they are in one direction. Next, this unidirectional fluence is expanded throughout the derived radiation field such that the fluence has the same value throughout the derived radiation field's volume. Using these two definitions, the ambient dose equivalent ${ }^{51}, H^{*}(d)$, is defined at a point in a radiation field as the dose equivalent that would be produced by the corresponding expanded and aligned field, in the ICRU sphere at a depth, $d$, on the radius opposing the direction of the aligned field. A depth 
of $d=10 \mathrm{~mm}$ is used throughout this work, as this is the depth recommended for use with the strongly penetrating radiation that is being simulated. For a known depth and radiation type, energy-dependent conversion coefficients can be evaluated that relate the fluence at a point to the ambient dose equivalent calculated at that point.

The equation for calculating ambient dose equivalent using conversion coefficients is:

$$
H^{*}(10) \doteq \int_{0}^{\infty}\left(\frac{d \bar{\Phi}_{\gamma}}{d E_{\gamma}}\right)\left(\frac{H_{\gamma}^{*}(10)}{\bar{\Phi}_{\gamma}}\right) d E_{\gamma}+\int_{0}^{\infty}\left(\frac{d \bar{\Phi}_{\mathrm{n}}}{d E_{\mathrm{n}}}\right)\left(\frac{H_{\mathrm{n}}^{*}(10)}{\bar{\Phi}_{\mathrm{n}}}\right) d E_{\mathrm{n}}
$$

where $\left(\frac{H_{\gamma}^{*}(10)}{\bar{\Phi}_{\gamma}}\right) \quad$ is the photon fluence to ambient dose equivalent conversion coefficient at a depth of $10 \mathrm{~mm}$

$\left(\frac{H_{n}^{*}(10)}{\bar{\Phi}_{n}}\right) \quad$ is the neutron fluence to ambient dose equivalent conversion coefficient at a depth of $10 \mathrm{~mm}$

This is the only method that calculates the important quantity, ambient dose equivalent. As is seen in Eq. (3.5), ambient dose equivalent conversion coefficients are used to weight the calculated average fluence. This fluence does not need to originate from inside any material, so it can be fluence from any location of interest. This means that unlike all the other methods discussed, this method can calculate a dose equivalent for a volume that is not tissue equivalent. The reason that a $10 \mathrm{~mm}$ depth is used is to allow for photoninduced electrons to build up so that a close-to-maximum dose is expressed by ambient dose equivalent. Because of how ambient dose equivalent is defined, it is straightforward to construct a physical detector to measure this quantity. As such, it is common to find area survey meters that report their measurements as ambient dose equivalent ${ }^{13}$. The value calculated from Eq. (3.5) is not as straight-forward to understand as it was in the previous 
methods. It calculates what would happen when the average fluence in a volume is used to calculate ambient dose equivalent. In other words, it calculates the ambient dose equivalent calculated for each point of the cell's volume, averaged over the volume.

In this work, ambient dose equivalent is calculated for cells of air, i.e., a dose equivalent is calculated for the case that a person is at a location where there is normally air. Since air and tissue attenuate radiation differently, ambient dose equivalent calculated for a cell cannot be interpreted as an average dose equivalent for that cell. Fortunately, uncharged particle attenuation in air is fairly low for volumes of air used in this work, so ambient dose equivalent should be close to being uniform over the volume of cells used. So, a way to look at Eq. (3.5) is that it calculates a quantity close to the maximum dose equivalent in a person should a person be somewhere within the cell.

By far, the biggest advantage of using this method to calculate ambient dose equivalent is that, unlike in the other methods, for the locations of interest, materials do not need to be changed to tissue equivalent. In the other methods, if a volume of the simulation is changed from air to tissue equivalent, it is expected that fluence will be significantly altered elsewhere within the simulation. So, for the other methods, at most, only a few locations can be calculated simultaneously before fluence becomes too perturbed to be meaningful. For this simulation method, simulations do not need to be changed from an empty-room state. Since no change of fluence occurs, it follows that the ambient dose equivalent at all applicable locations in the simulation can be calculated at the same time. This is a tremendous speed advantage for this method if one is looking to measure ambient dose equivalent at many locations within the same simulation.

One may notice the absence of the $E_{\text {kill. } \gamma}$ term in Eq. (3.5). The reason that this term is not included is twofold. The first reason is that it is difficult to accurately relate the dose 
due to killed photons in an arbitrary medium to the dose that would occur $10 \mathrm{~mm}$ deep in a tissue equivalent sphere, especially without any spectral information. The second reason is that photons below an energy of $10 \mathrm{keV}$ deposit at least $99 \%$ of their energy before reaching a depth of $10 \mathrm{~mm}$ in tissue equivalent material. In this work, a $10 \mathrm{keV}$ photon energy cutoff is used, and the photon spectra at all calculated locations have significant components well above $10 \mathrm{keV}$. Because of this, it can be reasoned that discarding the $E_{\mathrm{kill} . \gamma}$ term will cause the ambient dose equivalent to be less than a percent underestimated-well within the target simulation accuracy.

\subsection{Variance Reduction for Monte Carlo Simulations}

Variance reduction in MC simulations alters how simulated particles interact in matter in order to make calculations converge to a higher precision more quickly than possible if interactions were modelled to be analogous to nature. Variance reduction techniques can either be statistically fair or statistically unfair ${ }^{10,6}$. Fair variance reduction techniques alter particle interactions so that they are different than what is observed in nature, but some compensation method is employed so that what is seen in nature is statistically reproduced. Unfair techniques alter particle interactions so that they are fundamentally different than what is observed in nature, and some information is lost.

MCNP provides four methods of both fair and unfair variance reduction techniques for one to use: truncation, population control, modified sampling, and partially-deterministic methods ${ }^{6}$. Truncation methods truncate parts of phase space that should not contribute significantly to the solution. Population control methods split or destroy particles in an effort to control the number of samples taken in various regions of phase space. Modified 
sampling methods alter the statistical sampling of a problem to increase the likelihood of sampling a specific region of phase space. Partially-deterministic methods circumvent the normal random walk process by using deterministic-like techniques.

Energy cutoff is a common example of an unfair truncation technique that stops the transport of particles once their energy falls below a set cutoff. Their energy is assumed to be deposited all at once when they are killed. This is obviously not what happens in nature, but, if the cutoff energy is low enough, the extra distance that the particles would have travelled causes a negligible difference in calculations. Killing the particles at a given cutoff allows the time that would have been spent following particles below the cutoff to be spent on transporting particles of a higher energy, allowing for more statistics to be collected.

One common compensation method is to assign particles a property called weight. This weight is used as a tool to account for changes in particle interactions by changing it appropriately during non-analogue events, and then scaling any score obtained by this weight. So, for appropriate scaling, the weight is set to the inverse of the amount of times a process is increased over what would happen in nature, making weight essentially the amount of virtual particles contained within a transported particle. Also, in order for this system to work, particle weights into and out of interactions must, on average, always be conserved. Suppose, for example, in order to improve precision it is determined that it would be best to split a particle in two, it follows that upon splitting the particle into two each of those particles would be given half of the original weight. When one of these half-weight particles finally produce a score, that score is then scaled by the particle's weight-so while there are now two particles where there was once one, each of the particles only counts as half of a real particle. 
A fair variance reduction technique that is of particular interest to this work is a weight window. This technique makes use of the weight property of particles. A weight window can be used to control the spread of weights that occur with other when non-analogue techniques compensate by adjusting the particle's weight. The weight window does this by splitting particles of too-high weight into multiple lower-weight particles, and by randomly killing particles of too-low weight and assigning the randomly killed weight to the surviving low-weight particles. In MCNP, the evaluation on whether a particle should be split, be left unchanged, or be randomly killed because of weight windowing can be made when a particle crosses a surface and/or is involved in a collision. The real power of weight windows is realized when multiple weight windows are distributed in energy and space within a simulation. By adjusting the parameters of the weight windows, one can bias the simulation as to what energies and locations should have the most particles run, improving the sampling and statistics for those energies/locations.

For a tutorial on how to make use of many of the variance reduction techniques available in MCNP, one is pointed to Booth ${ }^{52}$. 


\section{Modelling of a Linac and Bunker}

The goal of this work is to show that Monte Carlo can be used to perform shielding calculations. In order to do this, we decided to show that meaningful MC calculations can be done for a bunker with a radiation source that matches the radiation pattern of a linac. This radiation pattern is rather complex due to the effect that the head components have on the incident electron beam. As a consequence, it was decided that the best way to produce a radiation pattern that most closely matches the complex one that is observed around a linac head would be to actually model a linac head. This portion of the modelling is described in Sec. 4.1. When the head modelling was finished, the radiation pattern around it was calculated, as described in Sec. 4.2. A bunker was then modelled, as is discussed in Sec. 4.3. Upon completion of the bunker model, the radiation pattern around the head was placed in the bunker model, and particle fluences and ambient dose equivalents were calculated at various locations inside and outside of the bunker, as described in Sec. 4.4. Next, a simulation to determine a renormalization factor that converts from per source particle to per dose under certain conditions was determined, as presented in Sec. 4.5. Finally, measurements and conventional calculations for comparison were completed, and they are presented in Sec. 4.6. Throughout all this work, MCNP 5.1.14 was used to perform the MC calculations. 


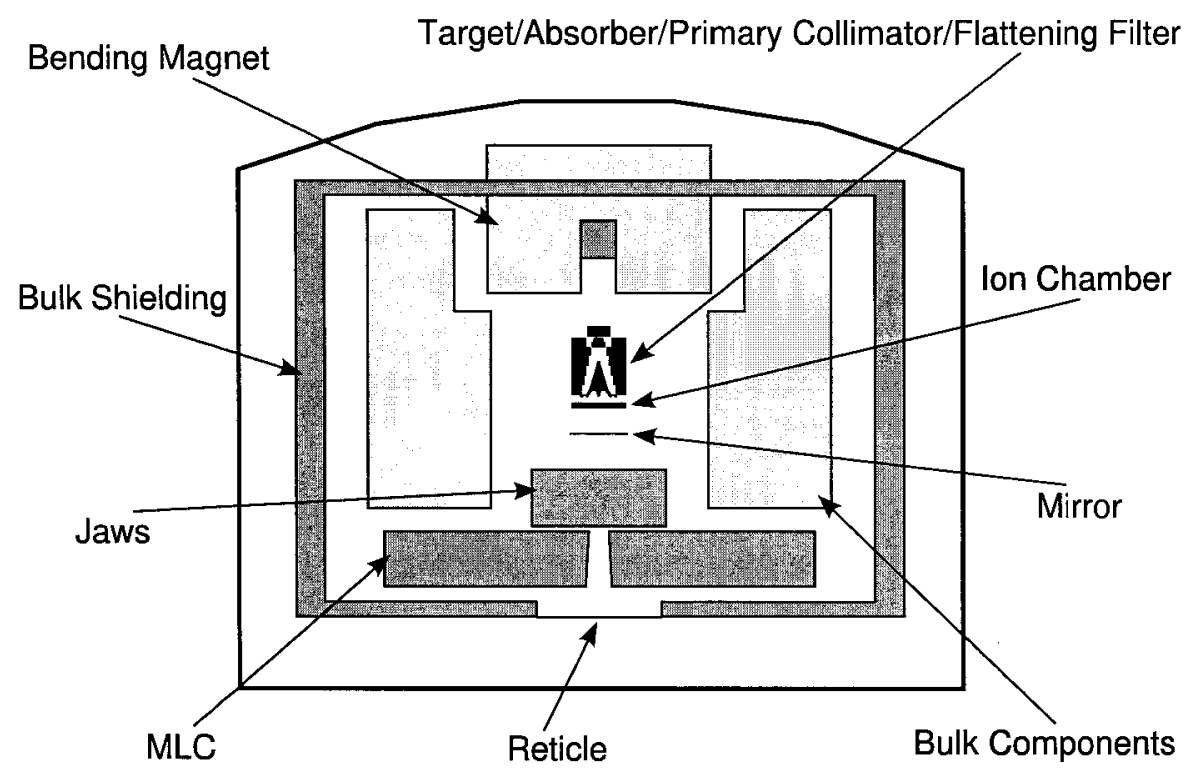

FIG. 4.1. A schematic cross-section of our model of a Siemens PRIMUS linac head. The important parts of the head are labelled. The outer box around the head is the boundary where the phase space was saved.

\subsection{Linac Head Modelling}

The choice of actual linac head modelled in this work should not change the ability to perform bunker shielding calculations. As the linac was free to be chosen, we selected an 18 MV Siemens PRIMUS linac - a linac that we have access to at our facility. From Siemens, we were able to obtain proprietary construction information concerning the beam forming components of an $18 \mathrm{MV}$ PRIMUS. This data was invaluable for modelling the head components. A schematic cross-section of the head as modelled is shown in Fig. 4.1.

The beam forming parts of the linac, i.e., the target, absorber, primary collimator, flattening filter, ion chamber, mirror, jaws and multileaf collimator (MLC), were relatively straightforward to model using the data provided by Siemens. All of the components were modelled with sub-millimetre accuracy as complex geometric combinations of planes, 
cylinders, and cones. It is known that the MLC should be arced, but attempts to do so resulted in the MLC and jaws occupying the same space in the model, which obviously does not happen in reality. So, without any more information on how the MLC is actually constructed, the Siemen's approximation that the MLC can be modelled as being flat was used for the model. Also, while the MLC is made of 29 closely-spaced pairs of metal leaves, it was modelled as a solid piece of tungsten for simplicity. The opening of the MLC and jaws were set to create a $10 \times 10 \mathrm{~cm}^{2}$ field size at the isocentre. This setting was chosen because it is a common setting, so it is easy to do comparisons with.

The initial electron beam was modelled according to the data given by Siemens. In general, incident electron beam properties supplied by manufactures are only considered as good starting points, and tuning of the data is done if precision is desired ${ }^{26}$. But, it was felt that this precise tuning would not improve the final calculated results because of the large unknown quantities elsewhere in the simulation. As a result, tuning of the electron beam parameters was not done - it was assumed that the reasonable values supplied would be sufficient for our purposes.

The data given by Siemens did not include any of the support structure around the beam forming components, nor any of the other parts in the head. From other work, it is known that the magnet and shielding are an important source of photoneutrons ${ }^{35}$; therefore, these two components needed to be estimated. A bending magnet from an older decommissioned Siemens linac was available, so approximate volumes of materials could be obtained. The bulk of the magnet was assumed to be iron, with a volume of approximately $18255 \mathrm{~cm}^{3}$, while a tungsten shield that went inside the magnet was measured to have a volume of approximately $645 \mathrm{~cm}^{3}$. 
The shielding was created in two parts. For the first part, it was assumed that there would at least be some shielding around the entire linac head. Consequently, a somewhat arbitrary shielding of lead was chosen: $2 \mathrm{~cm}$ thickness at the top and bottom of the head, and $4 \mathrm{~cm}$ along the side of the head. For simplicity of modelling, the bending magnet was split by the lead shielding, although this was done with care, making sure to keep the magnet's volume constant. When a simulation was run with this head model, there was notable difference between calculated relative photon doses around the head and measured values. From measurements taken during installation of the Siemens PRIMUS linac, discussed in more detail in Sec. 4.6.2, it was observed that the photon dose above the linac's head was approximately twice as large as the dose around the side of the head. So, extra bulk components were added to attempt to match photon doses better. These bulk components were designed out of a steel that was used in the beam forming components. The bulk shielding ended up having a volume of $121236 \mathrm{~cm}^{3}$.

The empty space in the head was filled with air with $1 \%$ moisture with a density of $0.0012 \frac{\mathrm{g}}{\mathrm{cm}^{3}}$. The air's composition by atomic fraction is: $1.00 \% \mathrm{H}, 0.02 \% \mathrm{C}, 77.26 \% \mathrm{~N}$, $21.26 \% \mathrm{O}$, and $0.46 \% \mathrm{Ar}$.

Finally, a surface that would enclose all of the head of a real linac was defined. It was designed so that it would be small enough that the surface would not hit the floor should it be rotated along with the head and objects could be placed in the linac beam close to the head, but large enough that all of the linac head would be enclosed if any extra detail was added in the future. This surface is what was used as the surface over which the phase space would be collected. Outside of the surface's boundaries, there is an infinite vacuum, i.e., once a particle leaves the box, it can never get back in. This vacuum is key for placing 
the head's phase space into another simulation as any particle that exits the boundary can not influence what is inside the boundary.

\subsection{Radiation Simulation in Linac Head}

To obtain a phase space from the linac head, MCNP was instructed to fully simulate the transport of electrons, photons, and neutrons. Next, biased photonuclear collision sampling was turned on. This allows for neutrons to be produced by photons, but in a way that more neutrons are produced than what is seen in nature such that neutron statistics can be improved. Specifically, the biasing splits photons in two at each collision, with one part undergoing a photoatomic event, and the other part a photonuclear event. The weight of each particle is adjusted by the ratio of their actual collision probability. Next, 214748364 initial electrons are set to be produced ( $\frac{1}{10}$ of the maximum source particles this release of MCNP supports). Finally, a weight window variance reduction technique is applied.

Three groups of weight windows were used: one group for electrons, one for photons, and one for neutrons. For estimating what weight windows should be used, the electron, photon, and neutron spectra were simulated for a $1 \mathrm{~m}^{2}$ area under the linac head. This was done prior to the addition of the bulk shielding and components. After obtaining the spectra, different weight window strategies were created for the different particle types, as described below.

For the electrons, it was decided that for any given energy, there should be an approximately equal number of electrons propagated. This allows for a general-use fluence, as all energies are equally represented. So, weight windows were chosen such that each energy's weight is proportional to the inverse of the electron population. In order to have a practical 
number of windows, windows were limited to when the targeted weights change by a factor of two, i.e., windows that passed weights of $1,2,4,8,16$, etc. were used. Also, the source electrons that are incident on the target should pass through the weight window. So, the weight window that the source electrons went through was set to pass electrons of unity weight--the same weight that the source electrons were started with. For reference, the source electron energy spectrum was modelled as a Gaussian with a mean of $14.28 \mathrm{MeV}$ and a full-width half-maximum of $1.5 \mathrm{MeV}$.

For the photons, much the same weight window strategy was followed as the one used for electrons. The main difference was where the weight window that passed unity weights was chosen. For this point, it was chosen to be at the high energy end of the observed photon spectrum, just before the spectrum starts to sharply drop off to zero. This was chosen so that a high-energy photon of near-unity weight, produced by a source electron of near-unity weight, does not undergo drastic weight windowing. This was done because drastic changes in particle weights can cause simulation problems.

Finally, for neutrons, a close to flat spectrum was observed. Consequently, one weight window was used for all energies below $1 \mathrm{MeV}$, mostly to keep weight variations low, and two others were used at higher energies to help amplify the higher-energy neutron component. Because biased photonuclear collision sampling was turned on, the neutrons being produced had a weight 10 to 100 times smaller than unity. So, a suitable weight was chosen such that the higher energy neutrons created by biased photonuclear collision sampling passed through the window as often as possible.

After the weight windows were appropriately set up, the simulation was set to run; collecting the phase space information from the boundary around the linac head. Because it was planned to do the bunker simulation with only photons and neutrons, only those parti- 
cles were saved in the phase space file. The simulation took approximately 4 days to run on an Athlon ${ }^{\mathrm{TM}}$ XP $2500+$, writing 370541 particle tracks to the phase space. Of the particle tracks written, 250551 were for neutrons and 119990 were for photons. Approximately $90 \%$ of the photon tracks are used to compose the treatment beam, while the neutrons are distributed fairly evenly around the head.

\subsection{Bunker Modelling}

We modelled a bunker in which an 18 MV Siemens PRIMUS linac is situated. The bunker's bulk shielding design specifications were available, which made modelling the bunker relatively straightforward. A horizontal cross-section of the modelled bunker is shown in Fig. 4.2.

The walls are made of concrete, although the exact composition and density is not known. As a result, it was decided that data for a generic concrete with a density of $2.3 \frac{\mathrm{g}}{\mathrm{cm}^{3}}$ would be used ${ }^{47}$. The generic concrete's composition by atomic fraction is: $16.88 \% \mathrm{H}$, $0.14 \% \mathrm{C}, 56.25 \% \mathrm{O}, 1.18 \% \mathrm{Na}, 0.14 \% \mathrm{Mg}, 2.14 \% \mathrm{Al}, 20.41 \% \mathrm{Si}, 0.57 \% \mathrm{~K}, 1.87 \% \mathrm{Ca}$, and $0.43 \% \mathrm{Fe}$. The steel reinforcing bar that is put in the concrete was not considered in the simulation. Within the walls, for a primary barrier, steel plates were used. Composition of this steel was taken from BEAMnrc's PEGS4 MILDSTEEL $700^{53}$. This steel has a density of $7.9 \frac{\mathrm{g}}{\mathrm{cm}^{3}}$, and a composition by atomic fraction of: $0.15 \% \mathrm{C}, 0.30 \% \mathrm{Si}, 0.50 \% \mathrm{Mg}$, and $99.05 \% \mathrm{Fe}$.

The door of the bunker is made out of 4 layers, from inside the bunker to outside:

1. $0.48 \mathrm{~cm}$ steel

2. $1.27 \mathrm{~cm} \mathrm{lead}$ 


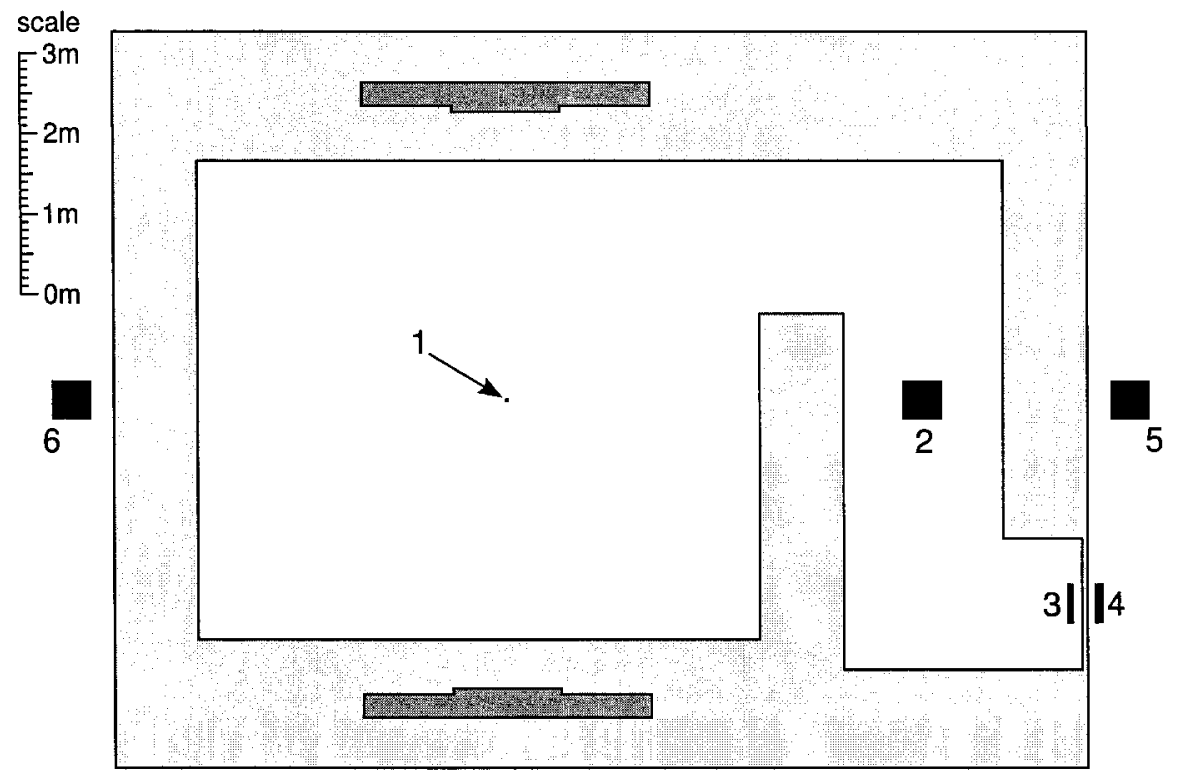

FIG. 4.2. A horizontal cross-section of the modelled bunker of an $18 \mathrm{MV}$ Siemens PRIMUS linac. The light grey areas are concrete, while the dark grey areas are steel. The numbered black boxes are the locations where ambient dose equivalent was calculated.

3. $5.08 \mathrm{~cm} 5 \%$ borated polyethylene

4. $0.48 \mathrm{~cm}$ steel

It was assumed that the steel for the door would be similar enough to the steel used in the walls to be able to use the same composition data. The $5 \%$ borated polyethylene, assuming $100 \%$ purity, has a composition by atomic fraction of: $64.89 \% \mathrm{H}, 2.66 \% \mathrm{~B}$, and $32.45 \% \mathrm{C}$. The density of the borated polyethylene is unknown, so the density of a commercially available $5 \%$ borated polyethylene was used. The borated polyethylene's density information was taken from Thermo Electron Corporation's similar borated polyethylene, Neutron Shielding Material Catalogue no. $201^{54}$, which has a density of $0.95 \frac{\mathrm{g}}{\mathrm{cm}^{3}}$. 
The free space of the bunker was filled with the same air with $1 \%$ moisture as the linac head. The floor of the the simulation was assumed to be made out of the same concrete as the walls of the bunker, and was set to be $1.5 \mathrm{~m}$ thick, below which is vacuum. The ceiling was made out of the same concrete as the walls, $1.22 \mathrm{~m}$ thick, with iron plates similar to the walls. Above the ceiling is $28 \mathrm{~cm}$ of air, then vacuum. Outside the bunker walls there is approximately a $2 \mathrm{~m}$ region of air, outside of which is vacuum.

\subsection{Radiation Simulation in Bunker}

To simulate the radiation in the bunker, first the phase space of the linac head was set to be propagated from appropriate surfaces. Relatively good results would likely occur if the space enclosed by the head's phase space boundary was filled with air. But since the linac head is quite large and dense, and more conveniently, was already simulated, it was included in the simulation of the bunker, inside the phase space in the same way it was for the head simulation. This way, particles that are redirected to the head from interactions within the bunker are fully able to "see" the simulated head. No efforts were made to simulate any other objects within the bunker though.

Originally, it was planned to have the linac's beam point directly at a wall, as this would allow us to look at the radiation transmitted through a wall in a worst-case scenario. The simulation was setup to allow for easy rotation of the linac head about the isocentre. But, for some reason, rotating the head from its designed position of pointing at the floor, resulted in MCNP not being able to track particles correctly, even though the geometry of the problem appeared correct. It is possible that this problem is related to MCNP 5 issue $9438^{55}$ : 


\section{Issue 9438: Particle tracking bug}

When a source point lies on a surface used elsewhere to define a cell of the problem, that cell seems to be invisible to the source particle, whether the cell is void or filled with a material. Also seen in MCNP 4C but the tallies are not zero; a nontrivial number of particles get lost.

If this is the actual issue, then a solution may be to redefine the geometry such that the phase space surfaces are not used as a boundary of the linac head. Since solving this issue could take a lot of time, and meaningful information could be obtained by leaving the head pointed at the floor, that is what was done.

Another shortcoming of this work is that a patient was not simulated in the beam. This is an issue because a patient is normally in the beam, and radiation is scattered off of patients. Depending on where in the bunker a shielding calculation is being performed, the patientscattered radiation may significantly increase the dose equivalent, changing the level of radiation that needs to be shielded.

For the bunker simulation, only neutrons and photons were simulated. Unlike the situation involving the linac head simulations, electrons were not needed as a source of radiation and simulation speed is greatly increased if electrons are not transported. It is expected that only small errors will be introduced by leaving out electron transport, especially since MCNP estimates the bremsstrahlung that would have been produced by electrons should they have been transported.

A strategy to apply variance reduction to the bunker was created. The largest problem that the bunker posed was its large attenuation of particles. Without any changes, thousands of source particles from the phase space could be run, and not a single one would be observed to penetrate the bunker walls. The solution was to use weight windows again, but 
instead of focusing on windowing energy, as was done for the head, the weight windows were used to amplify the particle population as it passed through bunker walls. Approximately every $10 \mathrm{~cm}$, photon radiation is expected to be halved in concrete ${ }^{7}$. So, weight windows were designed to amplify the particle population by 2 for every $10 \mathrm{~cm}$ that it travelled in concrete toward the outside of the bunker. This kept a near constant amount of particles travelling through the walls at all depths. The interior maze wall and the maze were given special consideration. Initial calculations showed that the photon population was about 100 times lower in the maze than the rest of the bunker. Therefore, it was decided that the interior maze wall, and the entrance to the maze from the interior of the bunker would increase the particle fluence by a series of 7 slabs of weight windows that double the particle population $\left(2^{7}=128\right)$. Again, multiple weight windows were used so that a smooth amplification of particles occurs. After considering the photon weight windows spatial distribution, it was found that the neutron population follows approximately the same population trends as the photon population in space. So, the same spatial weight window distribution pattern was used for neutrons as was used for photons. Reviewing the energy distribution of the neutrons and photons at various locations throughout the simulation, showed that the energy distribution of the weight windows could be kept the same as those that were used for the linac head. Consequently, this was done.

Locations to calculate dose equivalents were chosen as shown in Fig. 4.2. Location 1, which is at the isocentre, was given dimensions of $5 \times 5 \times 5 \mathrm{~cm}^{3}$, Locations 2,5 , and 6 were given dimensions of $50 \times 50 \times 50 \mathrm{~cm}^{3}$, while locations 3 and 4 were given dimensions of $10 \times 50 \times 50 \mathrm{~cm}^{3}$. These dimensions were chosen as a compromise between sampling more particles over a larger volume for better statistics, and being a small enough volume so that results for specific locations could be observed. All of the locations were centred vertically 
with the height of the isocentre. Locations 5 and 6 were placed $30 \mathrm{~cm}$ from the walls, as it is suggested that it is very uncommon for anyone to approach a bunker wall closer than that ${ }^{7}$. Locations 3 and 4 were placed $10 \mathrm{~cm}$ from the door, because it was thought that this would give more insight as to what types of radiation the door needs to shield, and how well the door actually does perform this shielding. If location 3 and 4 were wider and further away from the doors, more effects from the walls would be seen, making locations 3 and 4 less useful for drawing conclusions about the door.

The method discussed in Sec. 3.3.3.2 was used for dose equivalent calculations. This method calculates a dose equivalent known as ambient dose equivalent. This method was chosen as it does not require electrons to be transported, and because it allows multiple measurements to be made within the same simulation at one time.

To run the simulation, the linac head's phase space was set to be reused as a source of particles 10 times in a simulation, effectively making it seem like 2147483640 initial electrons were run ( 7 particles less than the maximum this release of MCNP supports). Comparing neutron and photon statistics showed that the photon spectrum had about an order of magnitude worse statistics. So, using the weight windows, an additional 10 times of particle reuse was set for photons. Similar to the linac head simulation, biased photonuclear collision sampling was turned on. This simulation took approximately 7 days to run on an Athlon ${ }^{\mathrm{TM}}$ XP $2500+$.

\subsection{Calculating a Common Reference for Simulations}

MCNP calculates data in terms of quantity per source particle. Consequently, for the bunker ambient dose equivalent calculations, results would be in ambient dose equivalent per 
source electron. For practical medical linac use, one does not specify how many electrons a linac should use. Instead, one specifies how many monitor units (MU) should be used. Monitor units are used as a reference level of radiation, and can be set to any reference a facility feels appropriate. MUs are set to be relative to the response of a radiation-measuring device in the linac. For the Siemens PRIMUS, the ion chamber in the beam is used to measure the radiation, and this is what a $\mathrm{MU}$ is set to be relative to.

At our facility, one MU is defined as the ion chamber response obtained under the following conditions:

- the beam is shaped as a square with an area of $10 \times 10 \mathrm{~cm}^{2}$ at the isocentre,

- the beam is directed downward,

- a $30 \times 30 \times 30 \mathrm{~cm}^{3}$ volume of water is placed horizontally centred at the isocentre, and is vertically aligned so that the top of the water is positioned $5 \mathrm{~cm}$ above the isocentre, and

- $1 \mathrm{cGy}$ of dose is deposited at the isocentre.

A simulation designed to measure source electrons per $\mathrm{MU}$ (as is defined at our facility), was created with the geometry discussed above. Because the linac head phase space did not include electrons, and dose calculations using electrons take a long time, a method to calculate dose without using electrons was, again, chosen. As ambient dose equivalent is not useful in this situation since we are dealing with dose to water, and not to tissue, this left one method to use: estimating dose as being equal to calculated kerma, as described in Sec. 3.3.3.1. Estimating dose as being equal to kerma is not always a suitable approximation, but at a $5 \mathrm{~cm}$ depth, it has been shown to be accurate within $2 \% 32$. 
Using similar methods as the ones used to calculate the linac head phase space and the ambient dose equivalent around the bunker, the dose to a $2 \times 2 \times 0.5 \mathrm{~cm}^{3}$ volume of water at the isocentre was calculated to be $(5.307 \pm 0.104) \times 10^{-16} \frac{\mathrm{Gy}}{\text { source } \mathrm{e}^{-}}$. This means that the conversion factor between MU and source electron is $(5.307 \pm 0.104) \times 10^{-14} \frac{\mathrm{MU}}{\text { source } \mathrm{e}^{-}}$. Extra precision was not sought, as it is known that the accuracy of this number is being compromised by the use of the kerma approximation and the use of an untuned incident electron beam. While both of these issues could be overcome, doing so would take significantly more time, and it is felt that the approximations used in modelling the bunker will introduce more significant errors than the errors observed here.

\subsection{Data for Comparison}

To be able to draw strong conclusions about how well our MC calculations can estimate dose equivalents, comparisons need to be made with other methods. Below, data from measurements around the linac head, in the maze, and outside the bunker are presented. Following that, expected results using the conventional method are presented.

\subsubsection{Measurements in the Maze and Outside the Bunker}

In order to verify that the MC calculations performed are realistic, photon and neutron measurements were taken. For our instruments, we used a Victoreen 451P for photon measurements, and a Victoreen $190 \mathrm{~N}$ for neutron measurements. The Victoreen 451P is a pressurized large-volume ion chamber survey meter that is relatively insensitive to neutrons. The Victoreen $190 \mathrm{~N}$ is a $\mathrm{BF}_{3}$ tube surrounded by a moderator assembly. It strongly 


\begin{tabular}{|c||c|c|c|}
\hline \multirow{2}{*}{ Location } & \multicolumn{3}{|c|}{ Dose Equivalent per MU $\left(\frac{\mathrm{mSv}}{\mathrm{MU}}\right)$} \\
\cline { 2 - 4 } & Photon & Neutron & Total \\
\hline \hline 2 & $(8.0 \pm 0.8) \times 10^{-6}$ & $(2.74 \pm 0.15) \times 10^{-5}$ & $(3.54 \pm 0.17) \times 10^{-5}$ \\
\hline 3 & $(7.2 \pm 0.4) \times 10^{-7}$ & $(1.03 \pm 0.06) \times 10^{-6}$ & $(1.75 \pm 0.07) \times 10^{-6}$ \\
\hline 4 & $(8.9 \pm 0.7) \times 10^{-8}$ & $(5.5 \pm 0.3) \times 10^{-8}$ & $(1.44 \pm 0.08) \times 10^{-7}$ \\
\hline 5 & $(7.3 \pm 1.7) \times 10^{-9}$ & $(5.2 \pm 0.7) \times 10^{-9}$ & $(1.25 \pm 0.18) \times 10^{-8}$ \\
\hline
\end{tabular}

TABLE 4.1. Dose equivalent per MU measured at various locations in the bunker.

rejects any photon signal. Together, these instruments can closely measure the photon and neutron dose equivalent components.

Using the above instruments, dose equivalent per MU was determined for many of the locations for which an ambient dose equivalent was calculated by Monte Carlo, as shown in Table 4.1. The locations are shown in Fig. 4.2. Two locations were not measured: location 1 (the isocentre), and location 6 (outside a thick bunker wall). These were not measured because at location 1 , the dose rate was too high for the instruments, while at location 6 , the dose rate was too low.

It should be mentioned that these measurements are not the exact same measurements as are calculated. First, they are done in a furnished bunker, which is expected to have different doses than the fictitious floating linac head in a bare concrete bunker simulation. Next, real instruments are used to measure dose equivalent, which perturb the field they're in, have small directional dependence, and need to be physically supported somehow. So, small differences between calculated and measured results are expected. Also, when taking measurements, only approximate locations were sought as it is expected that the measured dose equivalents are not strongly position-dependent. Finally, the door posed a small measurement problem. Instead of being one large door, it is a split door that has a 


\begin{tabular}{|c||c|c|}
\hline \multirow{2}{*}{ Region of Head } & \multicolumn{2}{c|}{ Photon Dose Equivalent per MU $\left(\frac{\mathrm{mSv}}{\mathrm{MU}}\right)$} \\
\cline { 2 - 3 } & Measured & Calculated \\
\hline \hline Top & $0.00480 \pm 0.00020$ & $0.0043 \pm 0.0003$ \\
\hline Around & $0.0024 \pm 0.0004$ & $0.00260 \pm 0.00010$ \\
\hline Bottom & $0.0036 \pm 0.0010$ & $0.0090 \pm 0.0005$ \\
\hline
\end{tabular}

TABLE 4.2. Photon head leakage per MU measured at various locations around the head compared to what was calculated from our model.

thick shielding piece behind the split. This split and shield could have significantly altered the dose equivalent calculated in front of, and behind the door. There was also the difficulty that the door opens into the room, so measurements in the centre of the door could not be done easily without irradiating an individual. Instead, measurements were done near the wall, behind the smaller of the split doors.

\subsubsection{Head Leakage Measurements}

During the installation of the Siemens PRIMUS linac at our facility, measurements of photon leakage around the linac's head were done. As measurements around the head are fairly similar, they can be distilled down to a few broad regions for comparisons, shown in Table 4.2. The top of the head refers to an area of the head directly opposite to the beam exit. Around the head refers to a band that goes around the linac head. The bottom of the head refers to the area near the beam exit, but not including the beam itself. The measurements were done with a large-volume ion chamber, as a fraction of the measured dose at the isocentre. In order to convert the measurement to be normalized to $1 \mathrm{MU}$ for comparisons, it needed to be assumed that the dose measured at the isocentre with the large-volume ion 
chamber would be approximately equal to the dose measured under the conditions used to define a MU at our facility (as discussed in Sec. 4.5).

To collect the calculated data shown in Table 4.2, a simulation was setup that measured ambient dose equivalent around the boundary of the linac head's phase space, as calculated in Sec. 4.2. Ambient dose equivalent was measured in similar broad regions as the measurements were distilled down to. As was designed, the dose equivalent per MU match very well at the top and around the head. The dose per MU at the bottom of the head is seen to be high. This is not too surprising as the measured values are taken at the patient plane $(100 \mathrm{~cm}$ from the target), while the computed values are taken at the edge of the stored phase space $(50 \mathrm{~cm}$ from the target). Taking into account the inverse-square law, the calculated results should be divided by 4 for comparison at the same distance. When this is done, the values are seen to be fairly compatible.

\subsubsection{Conventional Method Calculation for Comparisons}

In order to draw comparisons between the conventional method for calculations and $\mathrm{MC}$ calculations, calculations were performed using the conventional method. Two points were chosen to perform calculations for: inside and outside the door (locations 3 and 4 in Fig. 4.2). These calculations can be found in Appendix B, with a summary shown in Table. 4.3. The reasons that these two locations were chosen was because the conventional method has a well-defined and well tested method for evaluating the dose equivalent at these locations, and these calculations allow one to evaluate how well the door attenuates radiation. 


\begin{tabular}{|c||c|c|c|}
\hline \multirow{2}{*}{ Location } & \multicolumn{3}{|c|}{ Dose Equivalent per MU $\left(\frac{\mathrm{mSv}}{\mathrm{MU}}\right)$} \\
\cline { 2 - 4 } & Photon & Neutron & Total \\
\hline \hline 3 & $3.1 \times 10^{-6}$ & $3.4 \times 10^{-6}$ & $6.4 \times 10^{-6}$ \\
\hline 4 & $1.48 \times 10^{-6}$ & $6.8 \times 10^{-8}$ & $1.55 \times 10^{-6}$ \\
\hline
\end{tabular}

TABLE 4.3. Dose equivalent per MU calculated using the conventional approach for the locations immediately inside and outside the bunker door. 


\section{Results of Simulations}

The data collected from the bunker simulation, discussed in Sec. 4.4, are presented in Sec. 5.1. Following that, in Sec. 5.2, there is a short discussion of the quality of the MC calculations, followed by a discussion of the results in Sec. 5.3. Finally, comparisons to measurements and calculations are done in Sec. 5.4.

\subsection{Data Collected from Simulations}

For each of the locations shown in Fig. 4.2, the calculated ambient dose equivalents are shown in Table 5.1. The table shows the photon and neutron components of the ambient dose equivalents, along with the total ambient dose equivalents. The errors shown only include the random statistical errors-errors from geometric approximations are not included. It is also assumed that the photon and neutron ambient dose equivalent components are approximately independent for the calculation of the total ambient dose equivalent error. Results have been converted from ambient dose equivalent per source electron to ambient dose equivalent per MU, using the conversion factor calculated in Sec. 4.5. The uncertainty of the conversion factor was not included in the uncertainties listed in Table 5.1; it was used only to scale the data. This way, relative comparisons between ambient dose equiva- 


\begin{tabular}{|c||c|c|c|}
\hline \multirow{2}{*}{ Location } & \multicolumn{3}{|c|}{ Ambient Dose Equivalent per MU $\left(\frac{\mathrm{mSv}}{\mathrm{MU}}\right)$} \\
\cline { 2 - 4 } & Photon & Neutron & Total \\
\hline \hline 1 & $(1.095 \pm 0.009) \times 10^{1}$ & $(1.37 \pm 0.13) \times 10^{-2}$ & $(1.096 \pm 0.009) \times 10^{1}$ \\
\hline 2 & $(6.10 \pm 0.23) \times 10^{-6}$ & $(5.55 \pm 0.09) \times 10^{-5}$ & $(6.16 \pm 0.10) \times 10^{-5}$ \\
\hline 3 & $(1.328 \pm 0.028) \times 10^{-6}$ & $(5.74 \pm 0.05) \times 10^{-6}$ & $(7.06 \pm 0.06) \times 10^{-6}$ \\
\hline 4 & $(3.37 \pm 0.10) \times 10^{-7}$ & $(2.61 \pm 0.10) \times 10^{-8}$ & $(3.63 \pm 0.10) \times 10^{-7}$ \\
\hline 5 & $(1.285 \pm 0.029) \times 10^{-8}$ & $(2.78 \pm 0.05) \times 10^{-9}$ & $(1.56 \pm 0.03) \times 10^{-8}$ \\
\hline 6 & $(2.3 \pm 0.6) \times 10^{-7}$ & $(2.61 \pm 0.16) \times 10^{-9}$ & $(2.3 \pm 0.6) \times 10^{-7}$ \\
\hline
\end{tabular}

TABLE 5.1. Ambient dose equivalent per MU calculated at various locations in the bunker.

lents can be more easily done. The observed photon and neutron fluence spectra at each of the locations shown in Fig. 4.2 are presented in Figs. 5.1-5.12. These fluence spectra are normalized to the fluence observed per source electron. This normalization allows one to observe what fraction of a source electron, on average, is responsible for a given part of the fluence spectra. 


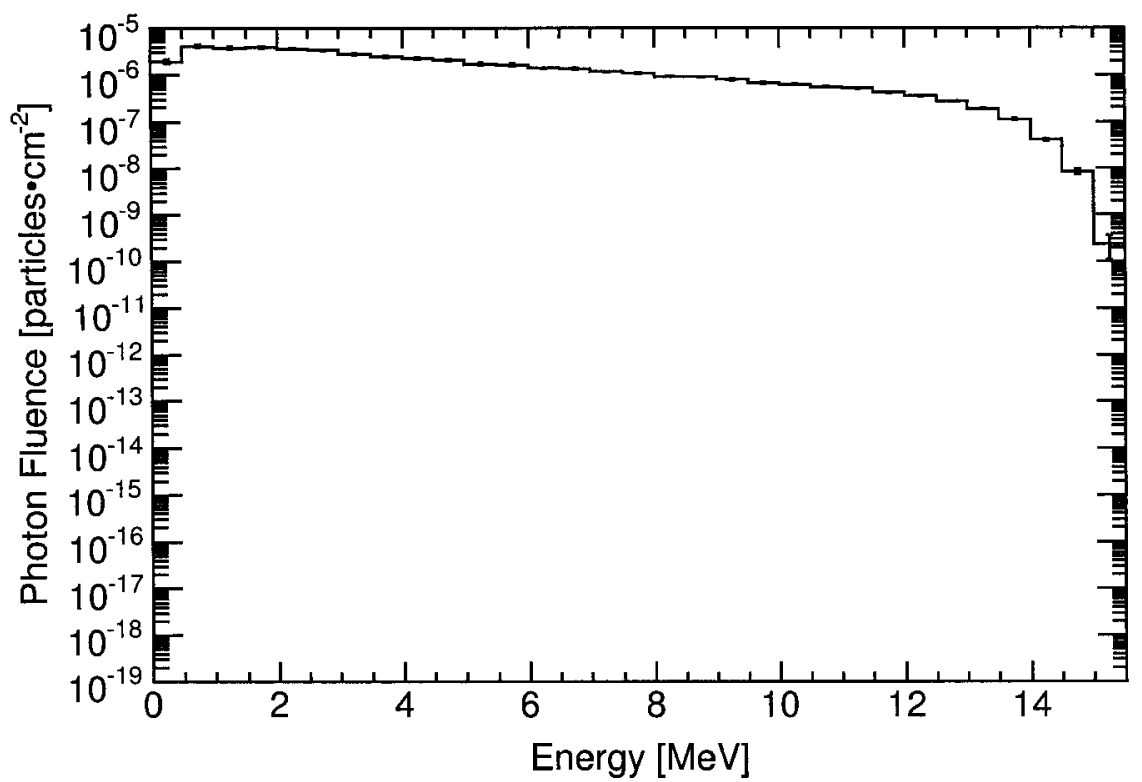

FIG. 5.1. Photon fluence at location 1: at the isocentre.

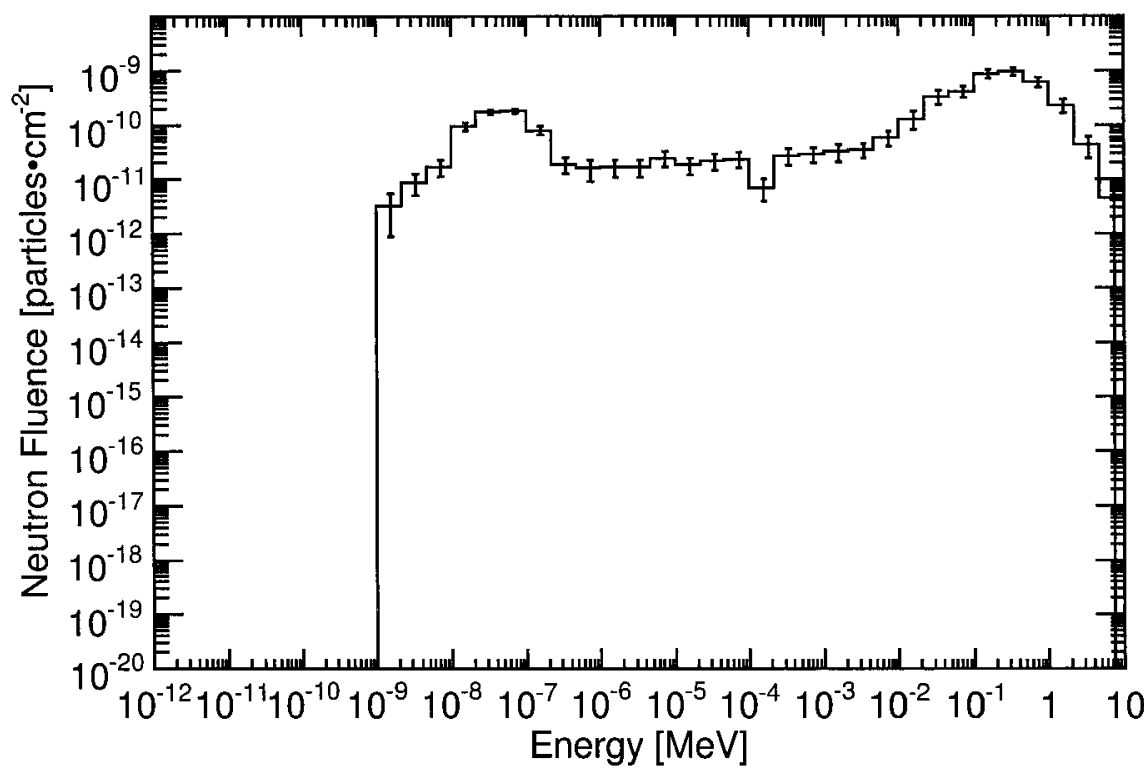

FIG. 5.2. Neutron fluence at location 1: at the isocentre. 


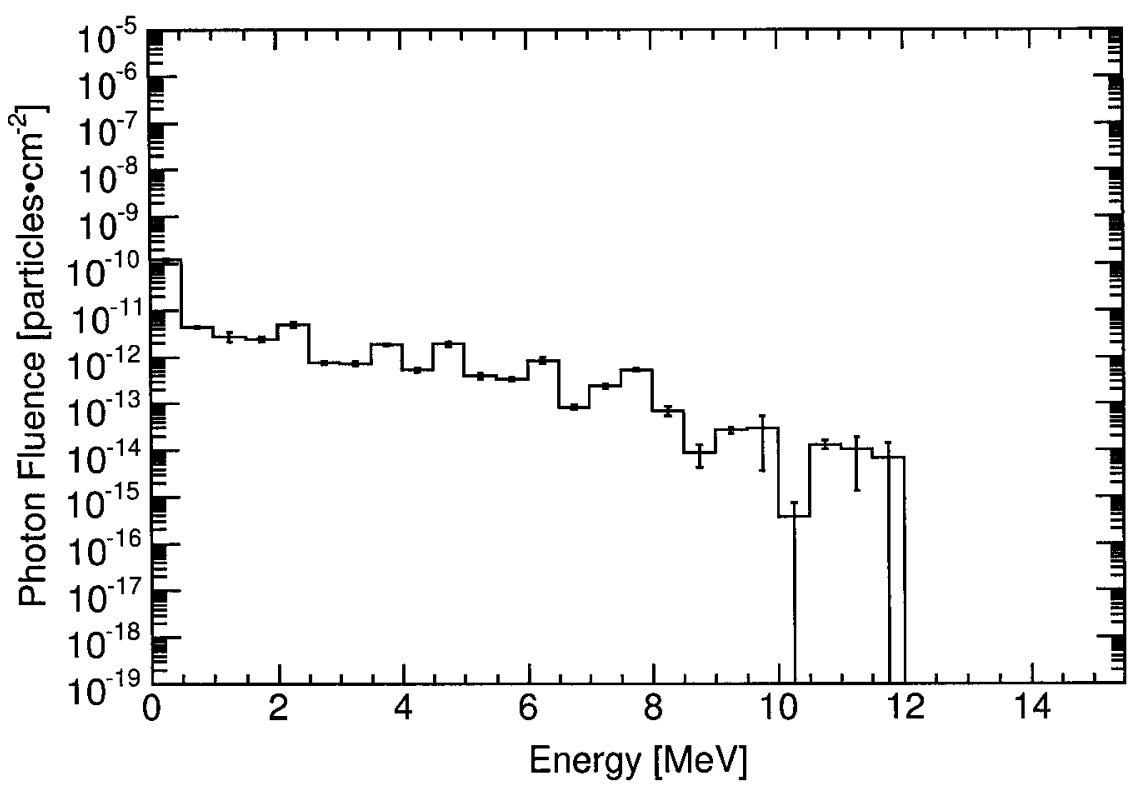

FIG. 5.3. Photon fluence at location 2: in the maze.

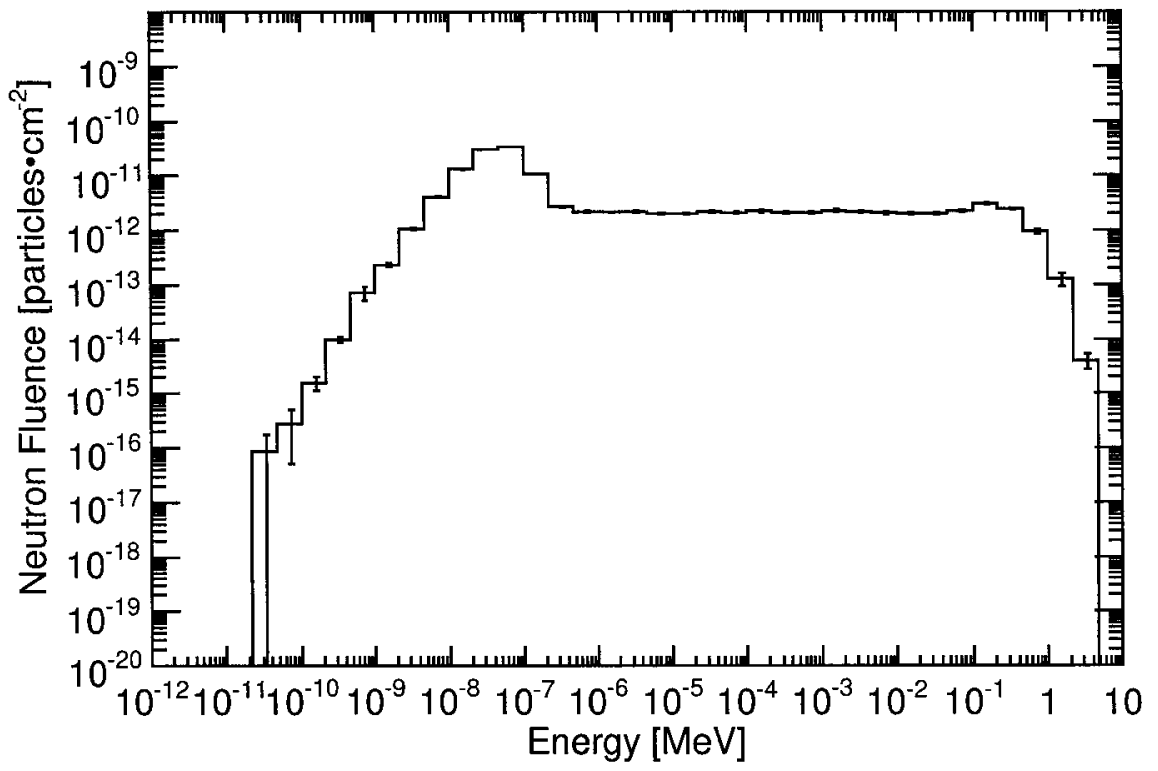

FIG. 5.4. Neutron fluence at location 2: in the maze. 


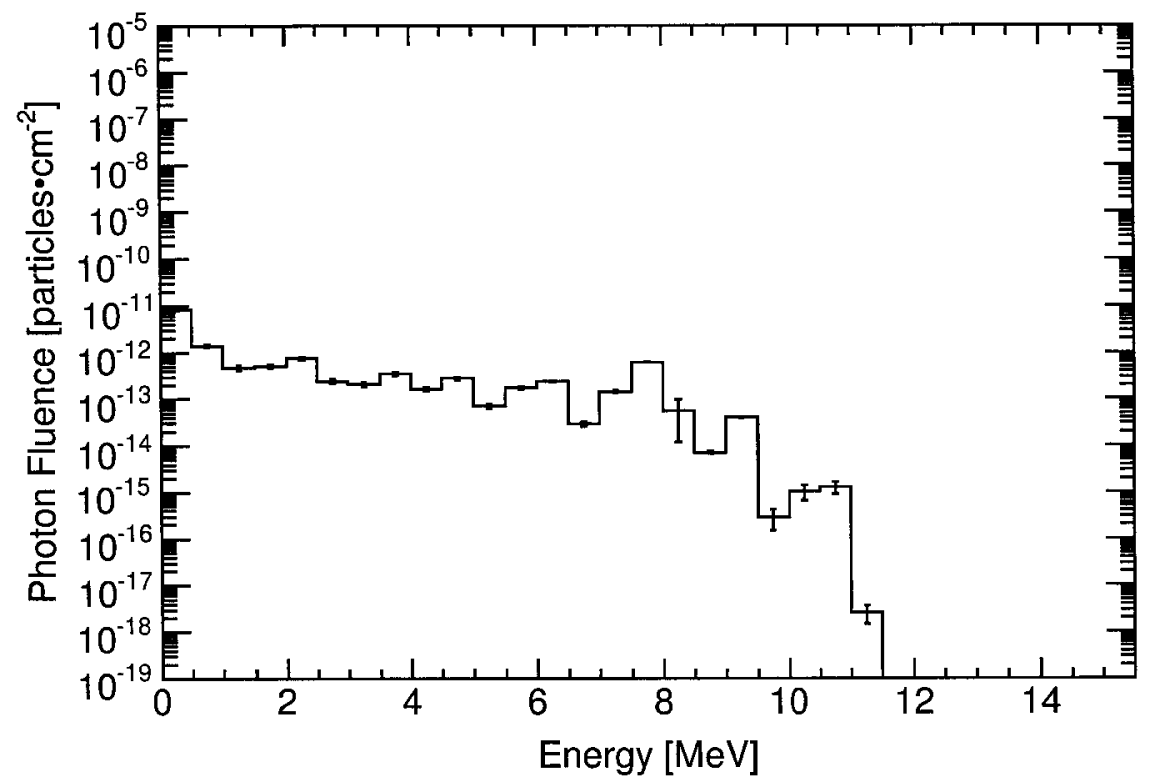

FIG. 5.5. Photon fluence at location 3: inside the door.

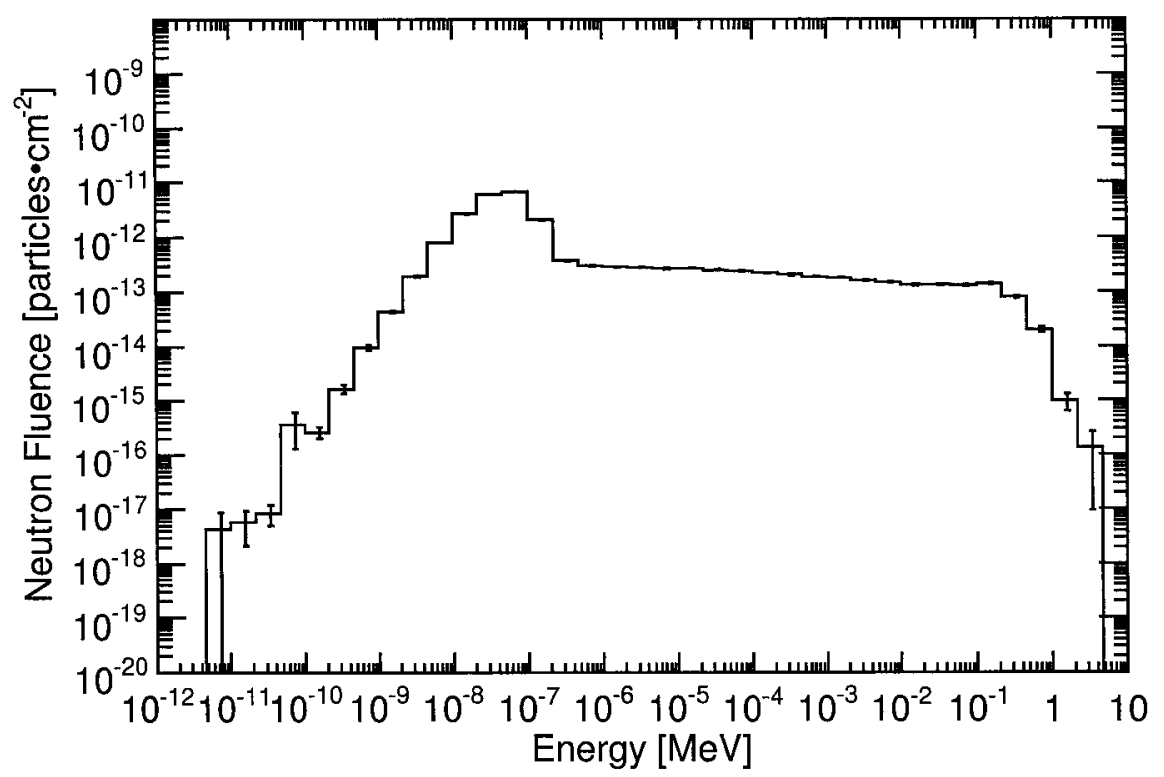

FIG. 5.6. Neutron fluence at location 3: inside the door. 


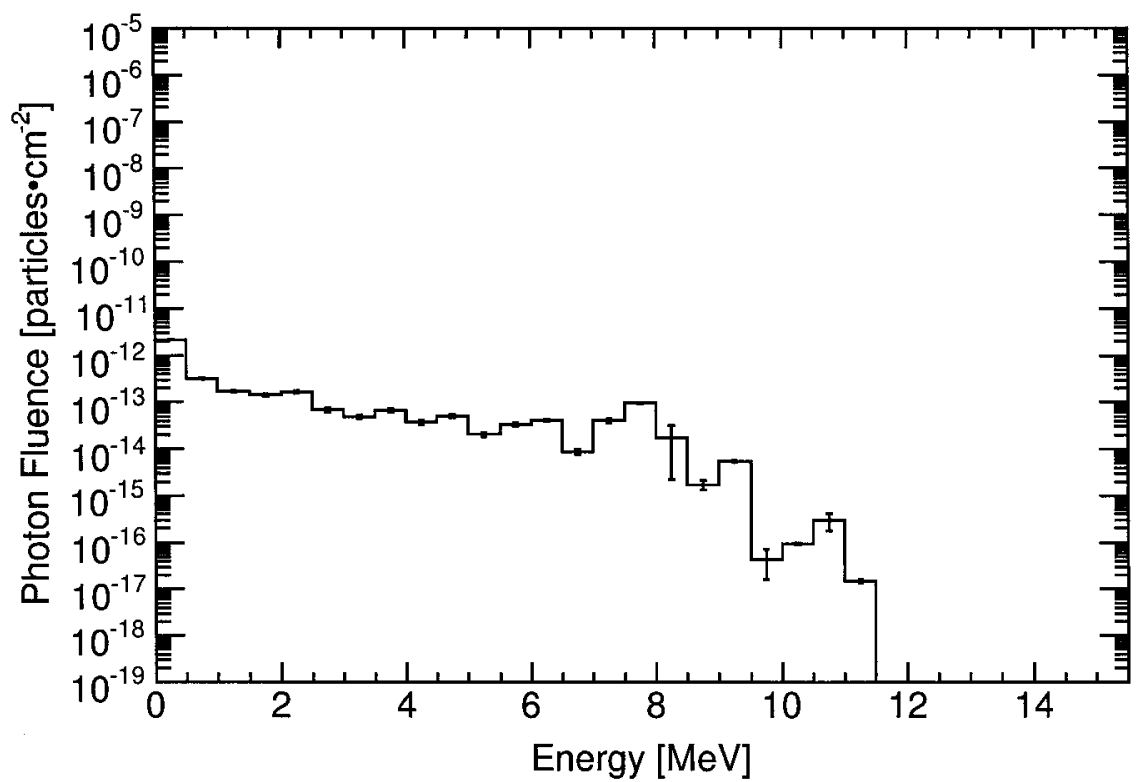

FIG. 5.7. Photon fluence at location 4: outside the door.

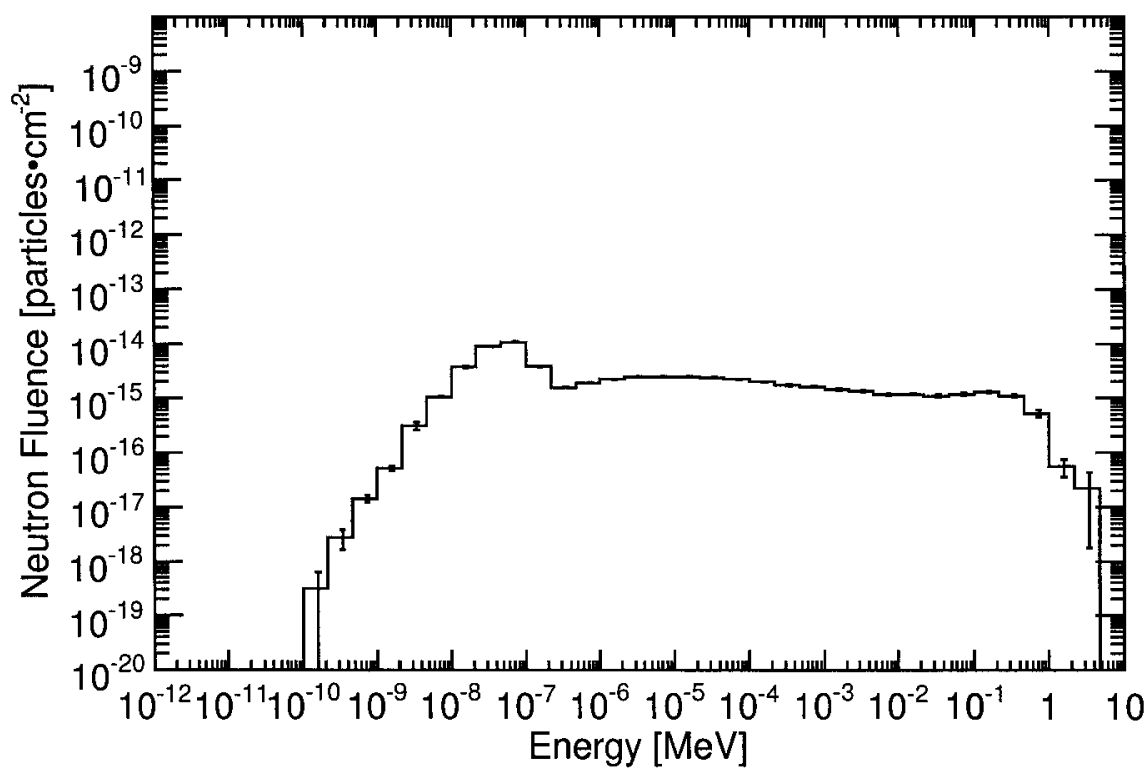

FIG. 5.8. Neutron fluence at location 4: outside the door. 


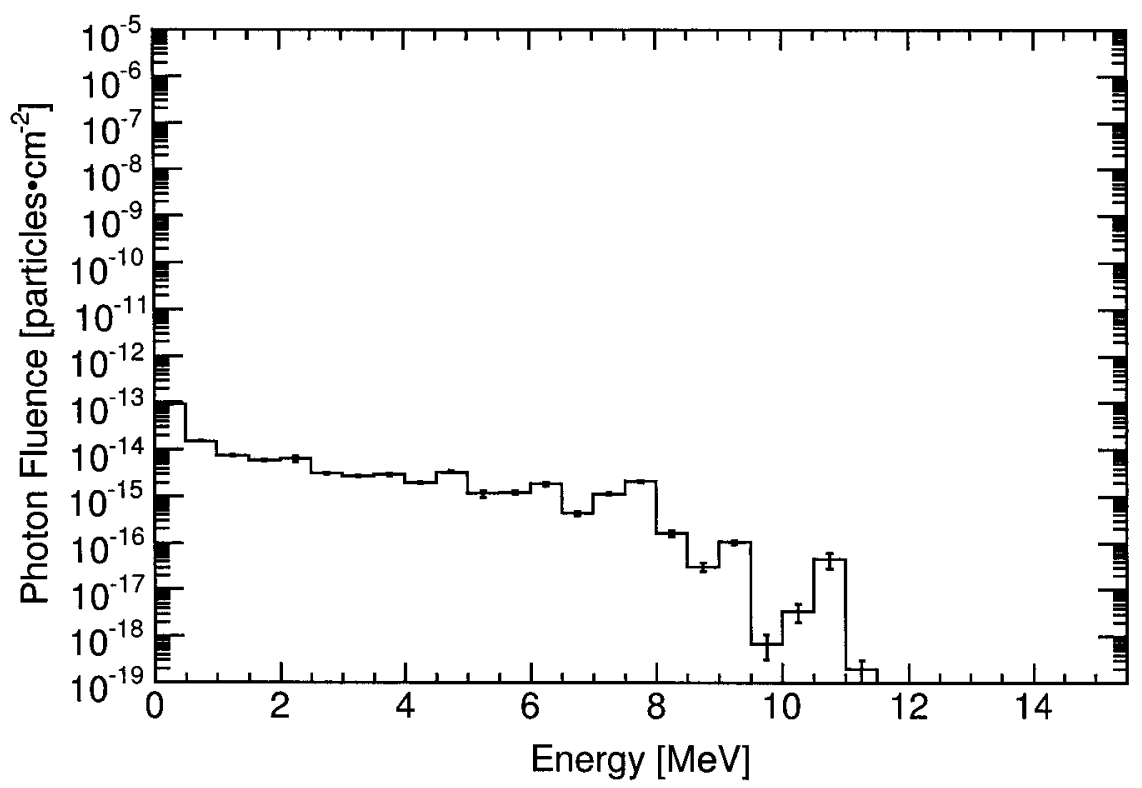

FIG. 5.9. Photon fluence at location 5: in the control area.

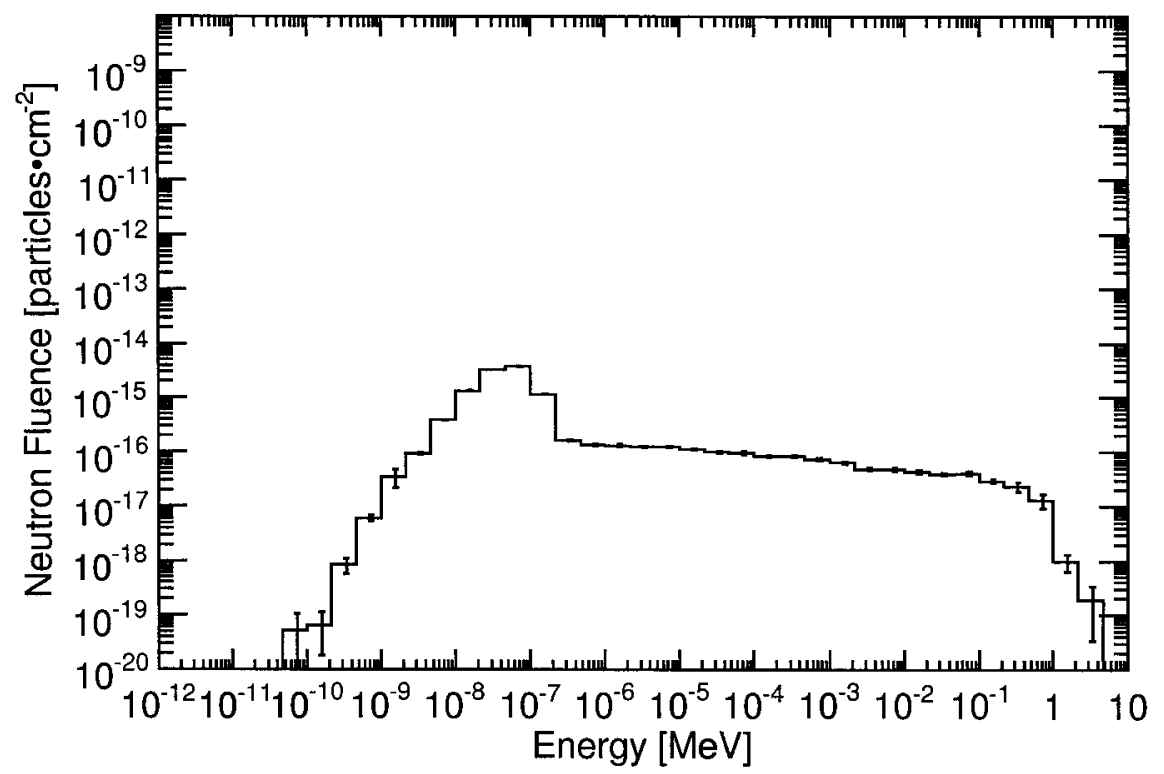

FIG. 5.10. Neutron fluence at location 5: in the control area. 


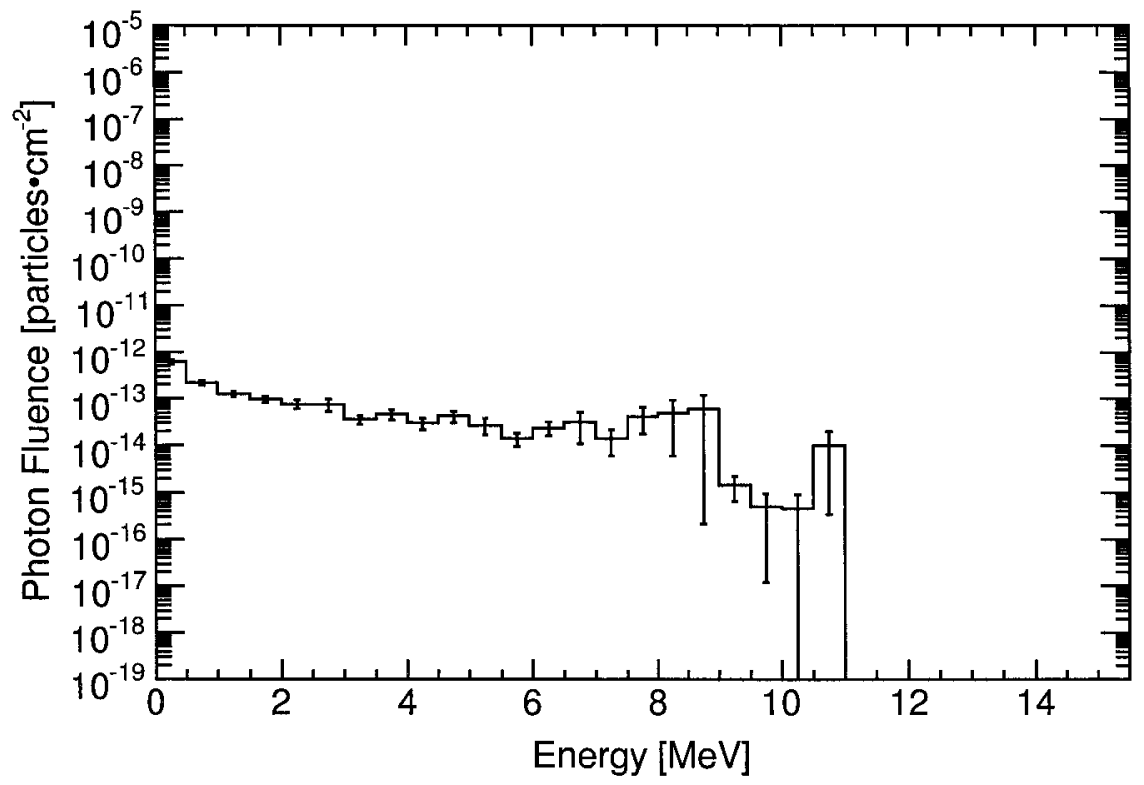

FIG. 5.11. Photon fluence at location 6: outside bunker, opposite the bunker maze side.

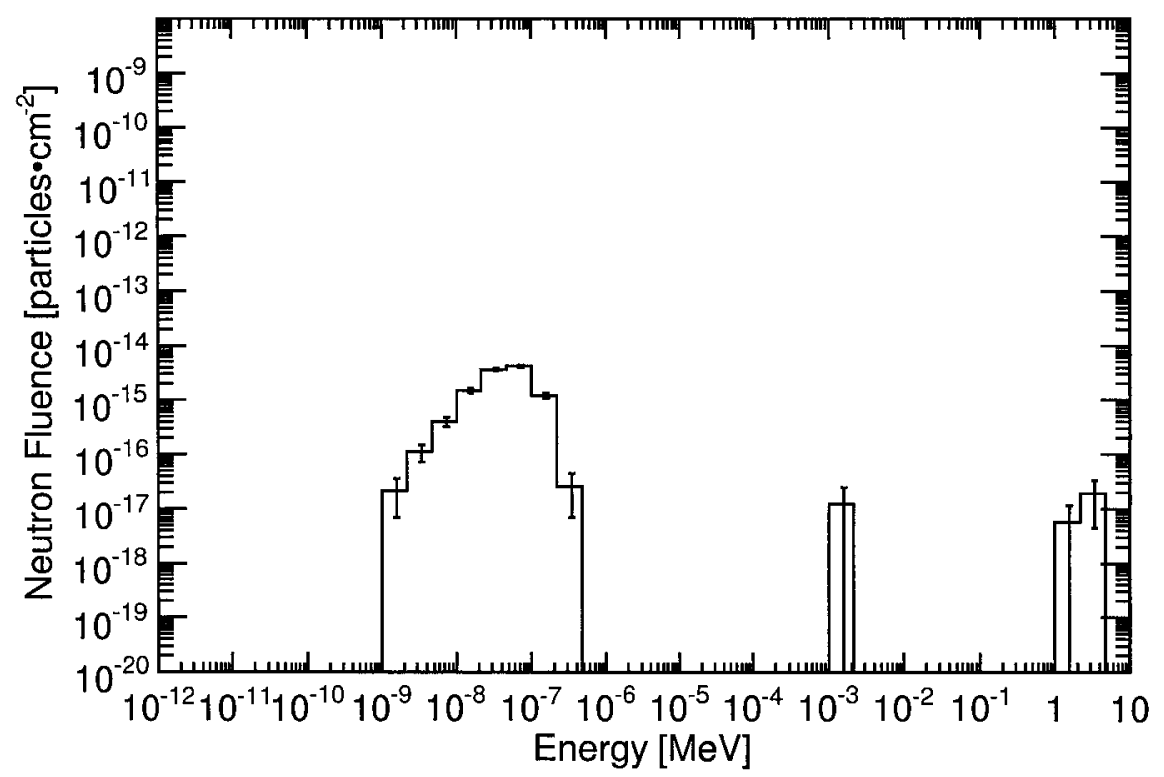

FIG. 5.12. Neutron fluence at location 6: outside bunker, opposite the bunker maze side. 


\subsection{Quality of Monte Carlo Calculations}

It is particularly hard to determine when MC simulations have properly converged on correct results. There is the possibility that a small region of great importance has not been properly sampled, and results are incorrect because of this. MCNP provides tools to help determine when a calculated result is likely to be correct ${ }^{6}$. Of particular interest to this work are:

- The trend of the mean value of a tally as a function of source particles-it is expected to converge to one number in a random fashion.

- The trend of the relative error of a tally as a function of source particles-it is expected to decrease as the square root of the inverse number of particles, and be reasonably small.

- The trend of the variance of the variance (VOV) of a tally as a function of source particles - it is expected to decrease as the inverse of the number of particles, and be reasonably small.

- How well the problem has been sampled. This can be evaluated by looking at the distribution of individual scores made to a tally. How reasonable the spread of individual scores is, and if enough data has been collected can then be judged. A quick check for this method is to calculate by how much the tally would change if the largest scoring event happened once more (which is an event that could occur when the next source particle is run). If this is a large change in the calculated value, it is conceivable that large fluctuations will occur in the tally, i.e., the tally has not yet stabilized. 
A tally is well behaved when the problem is being properly sampled, and the above trends are observed to be followed. When a well behaved tally acquires enough statistics for the precision sought, it is said to be converged. What follows is a concise analysis of the quality of the tallies used to calculate the results of Table 5.1. All of the data on which the following conclusions are based is not repeated here as it would take up much space, and not be very informative. Instead, highlights are presented.

\subsubsection{Quality of Location 1 Calculations}

In location 1 , the photon component appears to have properly converged. The neutron component looks to be well behaved, but if the largest scoring event happened once more, the mean would be higher by a factor of 15 . Therefore, more particles should be run to be sure that this tally has indeed converged.

\subsubsection{Quality of Location 2 Calculations}

The photon component's statistics indicate that the result is possibly not well converged. Also, Fig. 5.3 indicates that some of the higher energy photons are not being properly sampled. The neutron component is fairly well behaved.

\subsubsection{Quality of Location 3 Calculations}

The photon component's relative error and variance of the variance are not constantly decreasing-it is somewhat likely that the result is not accurate. The neutron component is well behaved. 


\subsubsection{Quality of Location 4 Calculations}

The photon component is similar to location 3; relative error and variance of the variance are not constantly decreasing-it is somewhat likely that the result is not accurate. The neutron component looks to be well behaved, but if the largest scoring event happened once more, the mean would be higher by a factor of 11 . Therefore, more particles should be run to be sure that this tally has indeed converged.

\subsubsection{Quality of Location 5 Calculations}

The photon component's statistics indicate that the result is possibly not well converged. The neutron component is fairly well behaved, but more statistics should be obtained to be sure.

\subsubsection{Quality of Location 6 Calculations}

The photon component indicates that not enough photons reached this detector to produce a reliable result. Also, Fig. 5.11 indicates that some of the higher energy photons are not being properly sampled. The neutron component indicates that not enough neutrons reached this detector to produce a reliable result. Also, Fig. 5.12 indicates that a large portion of the neutron energy spectrum is not being properly sampled.

\subsection{Discussion of Results}

From Sec. 5.2, it is clear that more particles should be run to obtain better statistics for accurate use of the calculated results. But, with the exception of location 6 , meaningful 
analysis and comparisons can still be made. Location 6 is obviously not being sampled correctly, so adjustments should to be made to the simulation in order to allow for more particles to reach this location.

The created model is not very realistic - it is a bunker with unfinished concrete walls, with only a floating linac head inside. Without modelling a "perfect" bunker for comparisons, it is rather difficult to judge what amount of uncertainty should be allocated to the calculated ambient dose equivalents for modelling inaccuracies. It is expected that the modelling approximations are the most significant source of error though.

The neutron spectra are expected to show a peak for the neutrons that have been thermalized after multiple collisions with matter. At room temperature $\left(20^{\circ} \mathrm{C}\right)$, the mean thermal energy is $0.025 \mathrm{eV}^{7}$, i.e., it is $2.5 \times 10^{-8} \mathrm{MeV}$, and their energy distribution should be Maxwellian ${ }^{5}$. This result is easily seen on each of the calculated neutron spectra, seen in Figs. 5.2, 5.4, 5.6, 5.8, 5.10, and 5.12.

Photoneutrons emitted from a linac have two primary components: direct emission neutrons and evaporation neutrons ${ }^{7}$. Both of these components produce neutrons with a mean energy of 1 to $2 \mathrm{MeV}$. This peak is indeed seen in the calculated results at the isocentre, as shown in Fig. 5.2. As neutrons lose energy through collisions, this high energy peak is reduced in comparison to the rest of the spectrum, and more neutrons become thermalized. This progression can be seen from Fig. 5.2 to Fig. 5.4, and finally to Fig. 5.10.

The photon spectrum at the isocentre in Fig. 5.1 has significant fluence up to about 15.5 MeV, while all the rest of the photon spectra show no photons with energy above $12 \mathrm{MeV}$. This is an expected result, as Compton scattering is the dominant photon scattering process at higher energies. In order for photons that are directed at the floor to be scattered back into the room, a significant drop of energy is needed. 
Of interest is that the statistical errors, shown in Table 5.1, when taken to be relative to their value are comparable in magnitude to one another. This is interesting because there are many orders of magnitude difference between values, yet the relative errors are nearly the same. This is largely due to the weight windows employed to amplify the amount of virtual particles in areas of low fluence such that statistics are better.

With the exception of Fig. 5.1, the rest of the photon fluence figures show incorrectly large jumps in the energy spectra. Reviewing the error bars around these jumps is further disconcerting, as it implies that it is statistically impossible for the spectra to be a smooth curve. This is surely not what happens in reality, as there is no physical reason for the jumps to appear. This artifact is most likely due to insufficient photons being sampled, and large particle amplifications occurring from phase-space reuse and many weight windows. Reviewing Sec. 4.2, it is seen that only about 10000 photons are saved in the phase space file for photon head leakage. Realizing that only a fraction of which will be directed at any region, possibly only 1000 of those saved photons will be responsible for the photon leakage statistics in one region. Because MCNP treats split particles as independent, and splitting in the 1000s of times is being done, this artifact appears. This artifact likely does not as strongly affect the calculated ambient dose equivalents though, as more averaging is done in this calculation. On the other hand, it would be reasonable to conclude that at least the photon ambient dose equivalent errors in Table 5.1 are being underestimated.

Looking at all of the observed fluence spectra, it appears that the higher-energy portion of the spectra are not as well sampled as the lower-energy portion, especially after penetrating barriers. In general, the higher the energy of a particle, the larger its fluence to ambient dose equivalent conversion coefficient is; thus, it is more influential on the calculation of ambient dose equivalent. Also, lower energy particles are born of higher energy particles. 
So, it is of interest to improve the sampling of the higher-energy portion of the fluence spectra. This can be done by further tuning the weight windows to preferentially amplify the number of particles of higher energy.

To put Table 5.1 in context, it is useful to determine a maximum acceptable dose equivalent per MU for shielding purposes. While unlikely, suppose that the linac will only ever be run at $18 \mathrm{MV}$, pointed at the floor, and with no patient, i.e., the conditions of linac use match this simulated work. If a maximum of $1 \frac{\mathrm{mSv}}{\text { year }}$ is accepted, and it is expected that the machine will deliver $3000000 \mathrm{MU}$ over the course of a year, and it assumed that all areas will be occupied with people, then it follows that a maximum of $3.3 \times 10^{-7} \frac{\mathrm{mSv}}{\mathrm{MU}}$ is allowable outside the bunker. With this number, it is seen that the control area (location 5) is within limits, while outside the door (location 4) is slightly too high.

\subsection{Comparison to Measurements and Calculations}

To evaluate how accurate our simulation is, we have compared it to measurements and other calculated results. Our simulation's results are first compared with results from other publications in Sec. 5.4.1. After that, our simulation's results are then compared to our own measurements and calculations in Sec. 5.4.2.

\subsubsection{Comparison with Published Work}

As our bunker design is unique, comparison of neutron dose equivalents to published measurements is difficult. Lin et al. ${ }^{43}$ have done measurements for a 15 MV Siemens PRIMUS ${ }^{43}$. Barquero et al. ${ }^{34}$ have also done measurements for an $18 \mathrm{MV}$ Siemens Mevatron KD-S. It is best to compare our calculated results for an $18 \mathrm{MV}$ Siemens PRIMUS to 
the 18 MV Siemens Mevatron KD-S, as the linacs are similar in design, and photoneutron yield is strongly energy dependent ${ }^{32}$. Because of the method used by Barquero et al. ${ }^{34}$, the neutron dose component in the primary beam could not be measured by them. At $25 \mathrm{~cm}$ from the isocentre, and with a $40 \times 40 \mathrm{~cm}^{2}$ beam, a neutron ambient dose equivalent of approximately $6 \frac{\mu \mathrm{Sv}}{\mathrm{MU}}$ was measured. From the work from Lin et al. ${ }^{43}$, it is plausible that this dose equivalent due to neutrons could be up to an order of magnitude larger within the primary beam. The calculated result of $(13.7 \pm 1.3) \frac{\mu \mathrm{Sv}}{\mathrm{MU}}$ is therefore reasonable compared to measured results. Barquero et al. ${ }^{34}$ also measured a neutron ambient dose equivalent of $0.1 \frac{\mu \mathrm{Sv}}{\mathrm{MU}}$ at the mouth of the maze, in a bunker shape that is not quite like the one that we did calculations for. Nevertheless, our calculated value of $(0.0555 \pm 0.0009) \frac{\mu \mathrm{Sv}}{\mathrm{MU}}$, being inside the maze, is quite reasonable. The photon ambient dose equivalent of $0.1 \frac{\mu \mathrm{Sv}}{\mathrm{MU}}$ at the mouth of the maze, measured by Barquero et al. ${ }^{34}$, does not compare very well to our calculated value of $(0.00610 \pm 0.00023) \frac{\mu \mathrm{Sv}}{\mathrm{MU}}$. This apparent disagreement could be in part due to their point of measurement being at the mouth of the maze, in a more direct path to measure leakage and scattered photons. Also, it appears that their method has a minimum sensitivity of approximately $0.1 \frac{\mu \mathrm{Sv}}{\mathrm{MU}}$ - the same number that was reported as the photon ambient dose equivalent.

The photon spectra at the isocentre can be compared to one calculated by Sheikh-Bagheri and Rogers ${ }^{27}$. They modelled an 18 MV Siemens KD linac using EGS4-a linac that uses much the same components as the $18 \mathrm{MV}$ Siemens PRIMUS modelled in this work. They spent much effort making sure their linac model reproduced a real photon beam. In their work, fluence as a function of energy is computed at the isocentre under the same conditions Fig. 5.1 of this work was calculated. When Sheikh-Bagheri and Rogers' ${ }^{27}$ work is plotted with the fluence shown in Fig. 5.1, Fig. 5.13 is obtained. Note that the fluence 


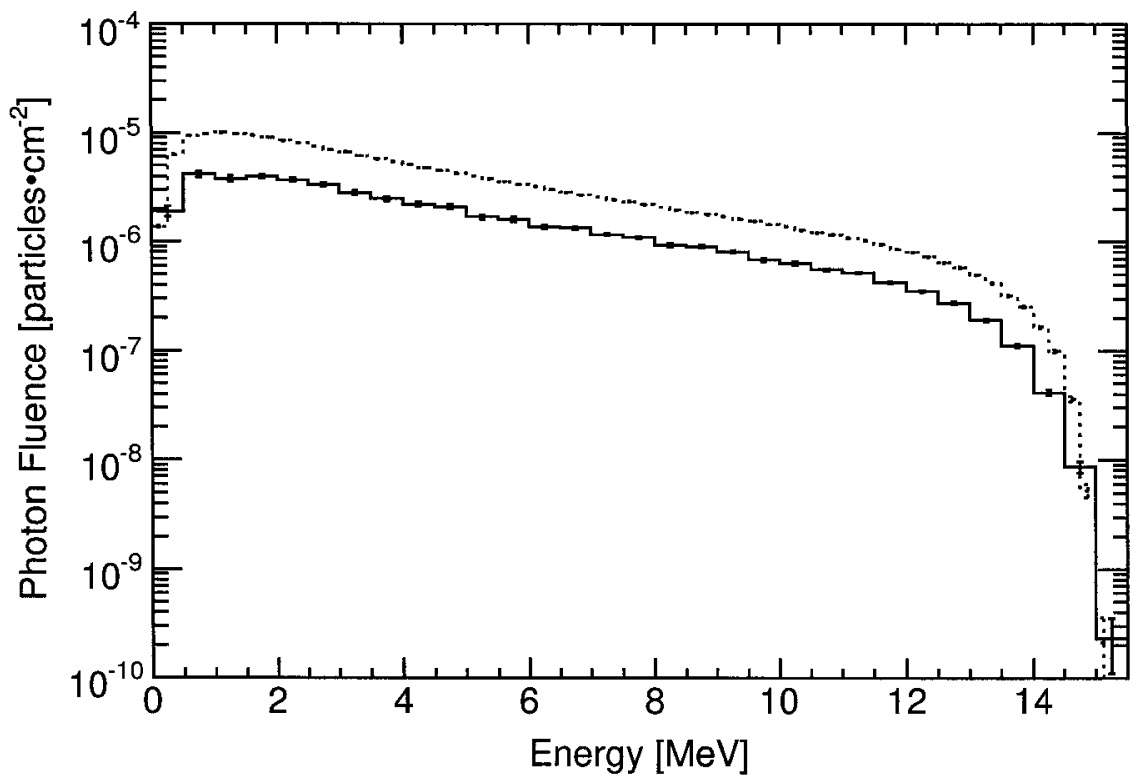

FIG. 5.13. Comparison of the photon fluence spectrum calculated by SheikhBagheri and Rogers ${ }^{27}$ (shown by dashed lines) and the photon fluence spectrum calculated in this work (shown by solid lines).

calculated by Sheikh-Bagheri and Rogers ${ }^{27}$ is done with $0.25 \mathrm{MeV}$ wide energy bins, compared to $0.5 \mathrm{MeV}$ wide bins used with this work. So, the fluence values of Sheikh-Bagheri and Rogers ${ }^{27}$ should theoretically be increased by a factor of two if the graphs are to be overlaid. An offset between the two plots is seen, and increasing the values of SheikhBagheri and Rogers ${ }^{27}$ by a factor of two would only accentuate the problem. Part of this could be due MCNP producing about 5\% less target bremsstrahlung than EGS4, as noted by Siebers et al. ${ }^{23}$, although the MCNP being studied in that paper is version $4 \mathrm{~B}$, while the version used in this work is version 5. The rest of the offset remains unexplained, although this author could be incorrectly interpreting what Sheikh-Bagheri and Rogers ${ }^{27}$ meant by, "Photon fluence spectra (photons per MeV per incident electron) ... for the radial bin $0 \leq r \leq 2.5 \mathrm{~cm}$." So, to compare the two spectra, Sheikh-Bagheri and Rogers" ${ }^{27}$ spectrum 


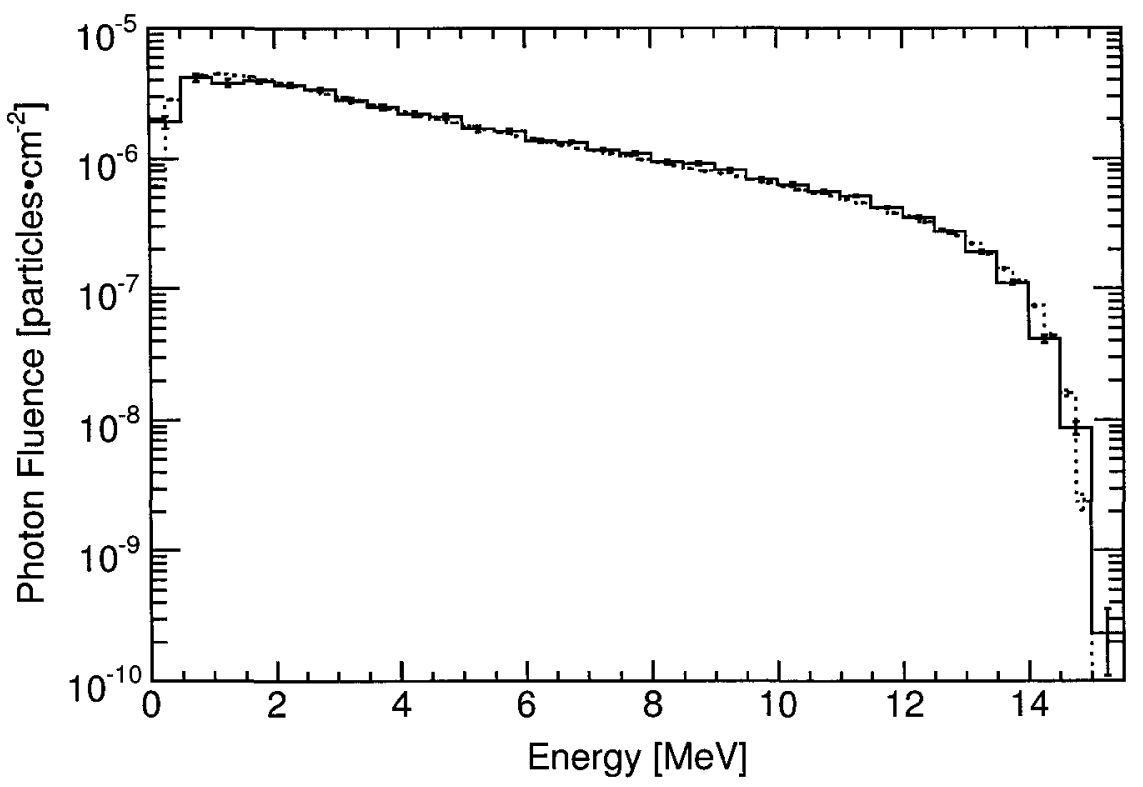

FIG. 5.14. Comparison of the photon fluence spectrum calculated by SheikhBagheri and Rogers ${ }^{27}$ (shown by dashed lines) and the photon fluence spectrum calculated in this work (shown by solid lines). The spectrum calculated by Sheikh-Bagheri and Rogers has been renormalized to have the same area as the spectrum calculated in this work.

was renormalized such that its area was equal to the area of the spectrum calculated in this work, as shown in Fig. 5.14. Looking at the two overlaid spectra, it is evident that the shapes of the spectra compare very well to one-another.

\subsubsection{Comparisons with Our Measurements and Calculations}

Comparisons between calculated values and measurements has been done in Table 5.2 in terms of dose equivalent ratios at locations inside and outside the bunker, as shown in Fig. 4.2. For benchmarking purposes, a comparison ratio has also been done for the conventional method calculations, shown in Table 5.3. From Table 5.2, the MC calculated values are seen to commonly be within a factor of 2 from the measured values, with a worst-case 


\begin{tabular}{|c||c|c|c|}
\hline \multirow{2}{*}{ Location } & \multicolumn{3}{|c|}{ Dose Equivalent Ratio } \\
\cline { 2 - 4 } & Photon & Neutron & Total \\
\hline \hline 2 & $0.76 \pm 0.08$ & $2.03 \pm 0.12$ & $1.74 \pm 0.09$ \\
\hline 3 & $1.84 \pm 0.12$ & $5.6 \pm 0.3$ & $4.03 \pm 0.17$ \\
\hline 4 & $3.8 \pm 0.3$ & $0.48 \pm 0.03$ & $2.52 \pm 0.15$ \\
\hline 5 & $1.8 \pm 0.4$ & $0.53 \pm 0.07$ & $1.25 \pm 0.18$ \\
\hline
\end{tabular}

TABLE 5.2. Ratio of Monte Carlo calculated ambient dose equivalent to measured dose equivalent at various locations in the bunker.

\begin{tabular}{|c||c|c|c|}
\hline \multirow{2}{*}{ Location } & \multicolumn{3}{|c|}{ Dose Equivalent Ratio } \\
\cline { 2 - 4 } & Photon & Neutron & Total \\
\hline \hline 3 & 4.2 & 3.3 & 3.7 \\
\hline 4 & 16.7 & 1.25 & 10.8 \\
\hline
\end{tabular}

TABLE 5.3. Ratio of conventionally-calculated dose equivalent to measured dose equivalent for the locations immediately inside and outside the bunker door.

of a factor of 5.6. Comparing to the conventionally-calculated data in Table 5.3, it is seen that factors range from 1.25 up to 16.7 . It is seen that the MC-calculated dose equivalents fluctuate above and below what the actual measured dose equivalent is. Conversely, the conventionally-calculated dose equivalents are always calculated to be above the measured dose equivalent (as they are designed to be).

An insightful comparison can be made by looking at the various door transmission factors, as is shown in Table 5.4. The transmission factors are simply the ratio of the dose equivalent outside the door to the dose equivalent inside the door. It is clear that the conventional method dose not properly attenuate the photon dose equivalent through the door, and as a result, the overall transmission factor is overestimated by a factor of approximately three. For the MC calculations, the neutron component is too strongly absorbed, while the 


\begin{tabular}{|c||c|c|c|}
\hline \multirow{2}{*}{ Evaluation } & \multicolumn{3}{|c|}{ Dose Equivalent Transmission Factor } \\
\cline { 2 - 4 } & Photon & Neutron & Total \\
\hline \hline Measurement & $0.123 \pm 0.012$ & $0.053 \pm 0.004$ & $0.082 \pm 0.006$ \\
\hline Conventional & 0.48 & 0.0203 & 0.241 \\
\hline Monte Carlo & $0.253 \pm 0.009$ & $0.00455 \pm 0.00018$ & $0.0513 \pm 0.0015$ \\
\hline
\end{tabular}

TABLE 5.4. Dose equivalent transmission factors for the bunker door as determined by measurements, conventional approach calculations, and Monte Carlo calculations.

photon component is not absorbed enough. It is possible that this is due to the absence of the use of neutron thermal tables in our simulations. These tables are used to to sample how a thermal neutron scatters with molecules and crystals ${ }^{6}$. If using neutron thermal tables increases the neutron transmission in the door, then less neutron capture gamma rays will be produced in the door, bringing the photon dose equivalent closer to what is expected. 


\section{Conclusions and Future Work}

A computer model of a linac head and a bunker has been successfully completed. Using Monte Carlo, the photon and neutron phase space around the head of the linac was calculated, with the radiation source being a modelled electron beam. This phase space was then used as a radiation source in the modelled bunker. Ambient dose equivalent and fluence spectrum calculations were done for various locations inside and outside of the bunker. With the exception of one location being clearly undersampled, the values calculated by our simulation are quite reasonable when compared to measurements, theory, and other calculations.

It took approximately 11 days of processing time to calculate the obtained results, ignoring modelling time. This was on a modest workstation with a single Athlon ${ }^{\mathrm{TM}}$ XP $2500+$ processor. Processors that are twice as fast as this processor are now commonly available, meaning that the calculations would take a mere 5.5 days if a current-day processor is used. If 10 such machines were setup to perform calculations in parallel, all of the MC calculation that were done in this work would be able to be done overnight. A computer setup like this would easily cost less than $\$ 10000$ today-less than $1 \%$ of the cost it currently takes to build one bunker. So, ignoring simulation setup time and expertise, it is possible to perform timely MC bunker shielding calculations. 
That being said, many of the results that were calculated would benefit from extra calculation time, so overnight may be optimistic with regards to the previous paragraph. On the other hand, some ways to improve the calculation efficiency have not been explored in this work. For example, it is possible to use the ring detector, a next-event estimator-type detector, in MCNP. It has been suggested that using this type of detector can improve calculation speed by a factor of ten to fifty ${ }^{36}$. It is also possible to bias the sampling of particle productions. For example, one could increase the probability of producing photons created as electron bremsstrahlung, increasing the efficiency of creating a neutron and photon phase space. Finally, the exponential transform, a modified sampling method, could be used to help neutrons and photons pass through the thick walls of a bunker. The exponential transform does this by allowing particle walks to move in a preferred direction by artificially reducing the macroscopic cross section in the preferred direction and increasing the cross section in the opposite direction 6 .

Future work might build upon this work by using Monte Carlo to explore how changes to the bunker shape and materials effect the measured doses outside the bunker. It could also include studying the effects of including various objects within the simulation, e.g., linac electronics, ceiling tiles, wood panelling, and room shelving. Other work may attempt to calculate effective dose-a dose weighted by dose to different tissues, requiring knowledge of where different tissues of a person are located at time of irradiation. More work might be to look at the effects of different beams types, field sizes, beam directions, and objects (e.g., a patient) in the beam. It is known that a patient in the beam can increase the photon dose at the door by $2-3$ times $^{38}$. Finally, an ambitious project would be to build a piece of software that is able to import engineering designs of a bunker, semi-automatically assign simulation properties and constraints, and automatically tune and adjust a succession of 
MC simulations, such that the output of the software is engineering designs perturbed to be more effective at shielding radiation.

Overall, this work shows that Monte Carlo can be used to simulate and test bunker shielding in a meaningful way and can easily become a very helpful future tool for bunker designers. 


\section{A Example MCNP Simulation Code for Tallying Dose Equivalent}

This appendix shows practical examples of how one would go about calculating dose equivalent using the four methods of dose equivalent calculations described in Sec. 3.3. To fully understand the following source code examples, the reader should reference the MCNP 5 manual $^{6}$. For these simplified examples, cell 1 will always be the cell that we are calculating dose equivalent for. This cell will be assumed to already be within radiation fluence, in a well-behaved simulation. For the cases where cell 1 needs to be filled with ICRU soft tissue, the following material definition can be used:

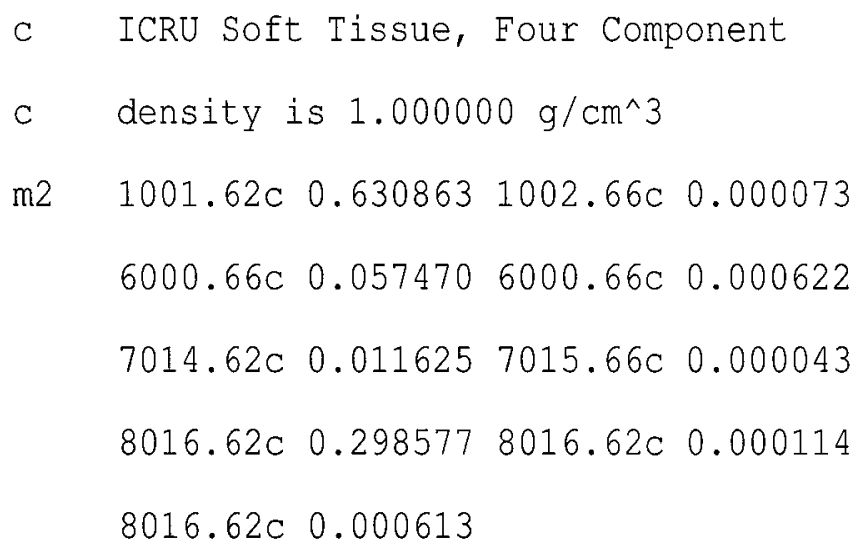


When needed, one can extract the volume and mass of cells from print table 50 of MCNP's output.

Energy killed for a particle type per source particle within a cell is not straight-forward to calculate in MCNP. The weight killed per source particle in a cell from energy cutoff can be obtained from print table 130 of MCNP's output. Unfortunately, the amount of energy killed within a cell per source particle is not available, although it is known that it must be somewhere between zero and the cutoff energy for each of the particles killed. The amount of energy and corresponding weight killed for the entire simulation is available in the problem summary of MCNP's output though, so the average energy killed per weight killed can easily be calculated. The weight killed per source particle in a cell can then be multiplied by the average energy killed per weight killed to obtain an estimate of the energy killed per source particle in a cell.

The values for neutron quality factors for the following MCNP code were obtained from Siebert and Schuhmacher ${ }^{56}$. The values for fluence to ambient dose equivalent conversion coefficients were obtained from ICRP publication $74^{2}$.

\section{A.1 Dose Equivalent Calculation from *F8:p,e and F6:n}

\section{Tallies}

To calculate dose equivalent per source particle from the *F8:p,e and F6:n tallies, the following MCNP tallying code would be used:

c calculate photon and electron energy deposition for cell 1

$\star f 118: p, e 1$ 
c calculate neutron kerma for cell 1

f116:n $\quad 1$

c multiply neutron kerma by Q

de116 lin $1 \mathrm{E}-9$ 1.58E-9 $2.51 \mathrm{E}-9 \quad 3.98 \mathrm{E}-9 \quad 6.32 \mathrm{E}-9 \quad 1 \mathrm{E}-8 \quad 2.53 \mathrm{E}-8$

$\begin{array}{lllllll}3.6 \mathrm{E}-8 & 6.3 \mathrm{E}-8 & 1.1 \mathrm{E}-7 & 2 \mathrm{E}-7 & 3.6 \mathrm{E}-7 & 6.3 \mathrm{E}-7 & 1.1 \mathrm{E}-6\end{array}$

$2 E-6 \quad 3.6 \mathrm{E}-6 \quad 6.3 \mathrm{E}-6 \quad 1.1 \mathrm{E}-5 \quad 2 \mathrm{E}-5 \quad 3.6 \mathrm{E}-5 \quad 6.3 \mathrm{E}-5$

$\begin{array}{lllllll}1.1 E-4 & 2 E-4 & 3.6 E-4 & 6.3 E-4 & 1.1 E-3 & 2 E-3 & 3.6 E-3\end{array}$

$\begin{array}{lllllllll}6.3 \mathrm{E}-3 & 0.011 & 0.02 & 0.036 & 0.063 & 0.082 & 0.086 & 0.09\end{array}$

$\begin{array}{llllllll}0.094 & 0.098 & 0.105 & 0.115 & 0.125 & 0.135 & 0.145 & 0.155\end{array}$

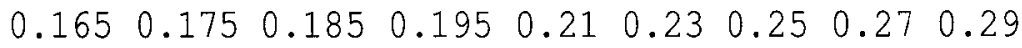

$\begin{array}{llllllllll}0.31 & 0.33 & 0.35 & 0.37 & 0.39 & 0.42 & 0.46 & 0.5 & 0.54 & 0.58\end{array}$

$\begin{array}{lllllllllll}0.62 & 0.66 & 0.7 & 0.74 & 0.78 & 0.82 & 0.86 & 0.9 & 0.94 & 0.98\end{array}$

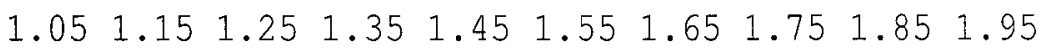

$\begin{array}{lllllllllllllllllll}2.1 & 2.3 & 2.5 & 2.7 & 2.9 & 3.1 & 3.3 & 3.5 & 3.7 & 3.9 & 4.2 & 4.6 & 5\end{array}$

$\begin{array}{llllllllllllllll}5.4 & 5.8 & 6.2 & 6.6 & 7 & 7.4 & 7.8 & 8.2 & 8.6 & 9 & 9.4 & 9.8 & 10.5\end{array}$

$\begin{array}{llllllllll}11.5 & 12.5 & 13.5 & 14.5 & 15.5 & 16.5 & 17.5 & 18.5 & 19.5\end{array}$

df116 lin $16.22 \quad 16.22 \quad 16.22 \quad 16.22 \quad 16.22 \quad 16.22 \quad 16.22 \quad 16.22$

$\begin{array}{llllllll}16.22 & 16.22 & 16.21 & 16.21 & 16.20 & 16.17 & 16.10 & 15.92\end{array}$

$\begin{array}{llllllllll}15.53 & 14.71 & 12.99 & 10.24 & 7.18 & 4.70 & 3.20 & 2.68 & 2.70\end{array}$

$2.95 \quad 3.43 \quad 4.16 \quad 5.23 \quad 6.74 \quad 8.98 \quad 11.81 \quad 14.97 \quad 16.51$

$\begin{array}{lllllllll}16.78 & 17.03 & 17.26 & 17.48 & 17.82 & 18.25 & 18.62 & 18.94\end{array}$

$19.20 \quad 19.42 \quad 19.60 \quad 19.75 \quad 19.87 \quad 19.96 \quad 20.06 \quad 20.15$

$20.17 \quad 20.16 \quad 20.12 \quad 20.05 \quad 19.96 \quad 19.86 \quad 19.75 \quad 19.64$

$\begin{array}{lllllllll}19.47 & 19.20 & 18.89 & 18.60 & 18.32 & 18.03 & 17.76 & 17.50\end{array}$ 


$$
\begin{array}{llllllllllll}
17.24 & 16.99 & 16.74 & 16.51 & 16.31 & 16.16 & 16.12 & 15.70 \\
15.03 & 14.58 & 14.16 & 13.66 & 13.28 & 12.99 & 12.58 & 12.36 \\
11.97 & 11.53 & 10.85 & 10.45 & 10.11 & 9.82 & 9.56 & 9.74 & 9.48 \\
9.26 & 8.72 & 8.49 & 7.82 & 7.80 & 6.92 & 6.92 & 6.78 & 6.81 \\
6.93 & 7.22 & 6.65 & 6.34 & 6.53 & 6.59 & 6.50 & 6.62 & 6.64 \\
7.11 & 6.88 & 7.12 & 7.41 & 7.56 & 7.47 & 7.44 & 7.47 & 7.46
\end{array}
$$

The calculated value of tally 118 is equal to $E_{\mathrm{D}}$, while the calculated value of tally 116 is equal to $\int_{0}^{\infty}\left(\frac{d \bar{K}_{\mathrm{n}}^{\prime}}{d E_{\mathrm{n}}}\right) Q_{\mathrm{n}}\left(E_{\mathrm{n}}\right) d E_{\mathrm{n}}$. Obtaining the value for $m$ is described in the beginning of this appendix. The calculation then follows Eq. (3.2).

\section{A.2 Dose Equivalent Calculation from F6:p and F6:n}

\section{Tallies}

To calculate dose equivalent per source particle from the F6:p and F6:n tallies, the following MCNP tallying code would be used:

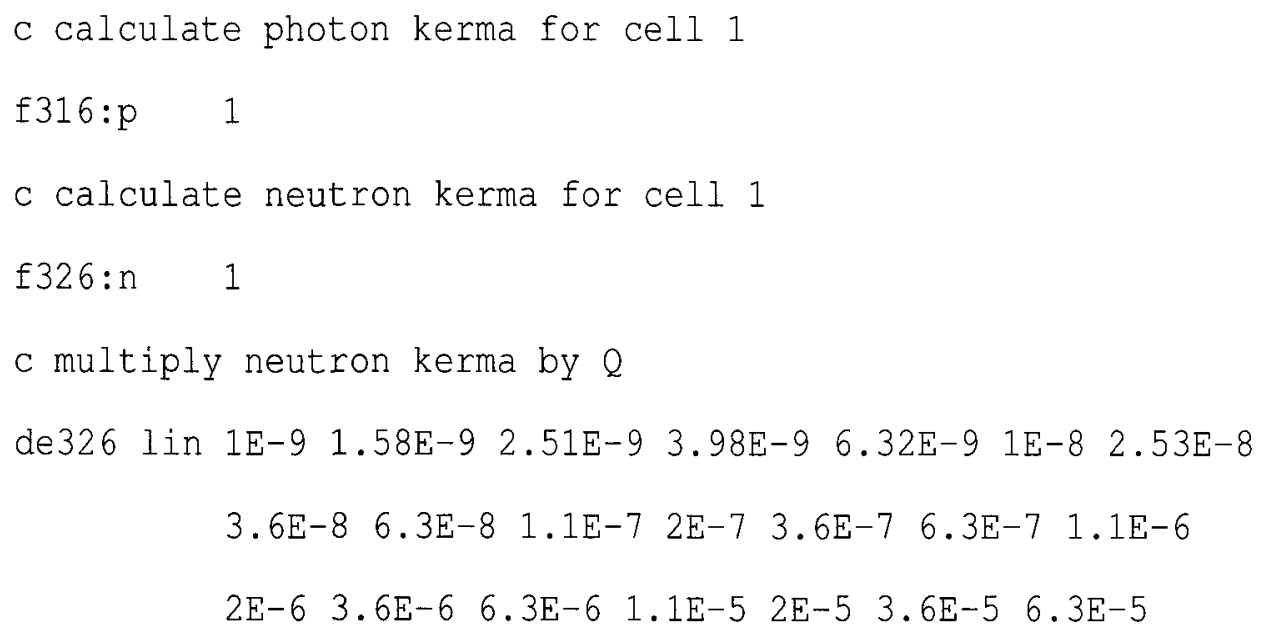




$$
\begin{aligned}
& \begin{array}{lllllll}
1.1 \mathrm{E}-4 & 2 \mathrm{E}-4 & 3.6 \mathrm{E}-4 & 6.3 \mathrm{E}-4 & 1.1 \mathrm{E}-3 & 2 \mathrm{E}-3 & 3.6 \mathrm{E}-3
\end{array} \\
& \begin{array}{lllllllll}
6.3 \mathrm{E}-3 & 0.011 & 0.02 & 0.036 & 0.063 & 0.082 & 0.086 & 0.09
\end{array} \\
& \begin{array}{llllllll}
0.094 & 0.098 & 0.105 & 0.115 & 0.125 & 0.135 & 0.145 & 0.155
\end{array}
\end{aligned}
$$

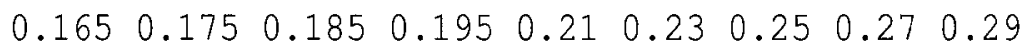

$$
\begin{aligned}
& \begin{array}{lllllllllll}
0.31 & 0.33 & 0.35 & 0.37 & 0.39 & 0.42 & 0.46 & 0.5 & 0.54 & 0.58
\end{array} \\
& \begin{array}{llllllllllll}
0.62 & 0.66 & 0.7 & 0.74 & 0.78 & 0.82 & 0.86 & 0.9 & 0.94 & 0.98
\end{array} \\
& \begin{array}{lllllllllll}
1.05 & 1.15 & 1.25 & 1.35 & 1.45 & 1.55 & 1.65 & 1.75 & 1.85 & 1.95
\end{array} \\
& \begin{array}{lllllllllllllllll}
2.1 & 2.3 & 2.5 & 2.7 & 2.9 & 3.1 & 3.3 & 3.5 & 3.7 & 3.9 & 4.2 & 4.6 & 5
\end{array} \\
& \begin{array}{lllllllllllllll}
5.4 & 5.8 & 6.2 & 6.6 & 7 & 7.4 & 7.8 & 8.2 & 8.6 & 9 & 9.4 & 9.8 & 10.5
\end{array} \\
& \begin{array}{llllllllll}
11.5 & 12.5 & 13.5 & 14.5 & 15.5 & 16.5 & 17.5 & 18.5 & 19.5
\end{array}
\end{aligned}
$$

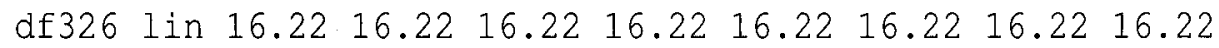

$$
\begin{aligned}
& \begin{array}{lllllllll}
16.22 & 16.22 & 16.21 & 16.21 & 16.20 & 16.17 & 16.10 & 15.92
\end{array} \\
& \begin{array}{llllllllll}
15.53 & 14.71 & 12.99 & 10.24 & 7.18 & 4.70 & 3.20 & 2.68 & 2.70
\end{array} \\
& 2.95 \quad 3.43 \quad 4.16 \quad 5.23 \quad 6.74 \quad 8.98 \quad 11.81 \quad 14.97 \quad 16.51 \\
& \begin{array}{lllllllll}
16.78 & 17.03 & 17.26 & 17.48 & 17.82 & 18.25 & 18.62 & 18.94
\end{array} \\
& 19.2019 .42 \quad 19.60 \quad 19.75 \quad 19.87 \quad 19.96 \quad 20.06 \quad 20.15 \\
& 20.1720 .16 \quad 20.12 \quad 20.05 \quad 19.96 \quad 19.86 \quad 19.75 \quad 19.64 \\
& \begin{array}{llllllll}
19.47 & 19.20 & 18.89 & 18.60 & 18.32 & 18.03 & 17.76 & 17.50
\end{array} \\
& \begin{array}{lllllllll}
17.24 & 16.99 & 16.74 & 16.51 & 16.31 & 16.16 & 16.12 & 15.70
\end{array} \\
& \begin{array}{llllllll}
15.03 & 14.58 & 14.16 & 13.66 & 13.28 & 12.99 & 12.58 & 12.36
\end{array}
\end{aligned}
$$

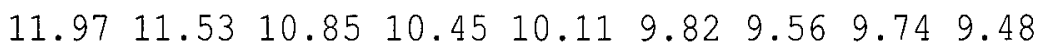

$$
\begin{aligned}
& \begin{array}{llllllllll}
9.26 & 8.72 & 8.49 & 7.82 & 7.80 & 6.92 & 6.92 & 6.78 & 6.81
\end{array} \\
& \begin{array}{llllllllll}
6.93 & 7.22 & 6.65 & 6.34 & 6.53 & 6.59 & 6.50 & 6.62 & 6.64
\end{array} \\
& \begin{array}{llllllllll}
7.11 & 6.88 & 7.12 & 7.41 & 7.56 & 7.47 & 7.44 & 7.47 & 7.46
\end{array}
\end{aligned}
$$


The calculated value of tally 316 is equal to $\int_{0}^{\infty}\left(\frac{d \bar{K}_{\gamma}^{\prime}}{d E_{\gamma}}\right) d E_{\gamma}$, while the calculated value of tally 326 is equal to $\int_{0}^{\infty}\left(\frac{d \bar{K}_{\mathrm{n}}^{\prime}}{d E_{\mathrm{n}}}\right) Q_{\mathrm{n}}\left(E_{\mathrm{n}}\right) d E_{\mathrm{n}}$. Obtaining the value for $m$ and $E_{\mathrm{kill}, \gamma}$ is described in the beginning of this appendix. The calculation then follows Eq. (3.4).

\section{A.3 Dose Equivalent Calculation from F4:p and F4:n Tallies}

To calculate dose equivalent per source particle from the F4:p and F4:n tallies, the following MCNP tallying code would be used:

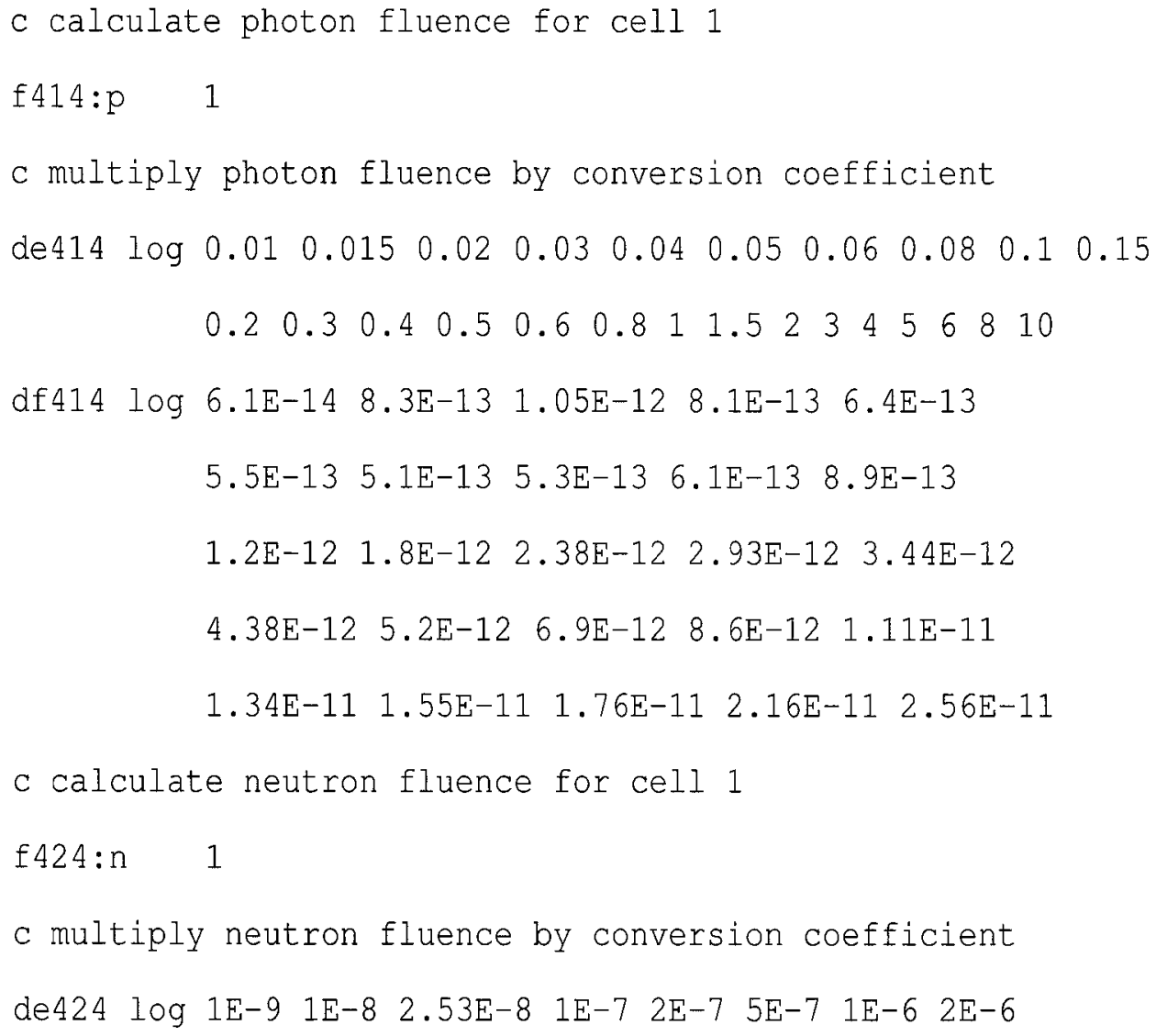




$$
\begin{aligned}
& \begin{array}{lllllllll}
5 E-6 & 1 E-5 & 2 E-5 & 5 E-5 & 1 E-4 & 2 E-4 & 5 E-4 & 1 E-3 & 2 E-3
\end{array} \\
& \begin{array}{llllllllll}
5 \mathrm{E}-3 & 0.01 & 0.02 & 0.03 & 0.05 & 0.07 & 0.1 & 0.15 & 0.2
\end{array} \\
& \begin{array}{lllllllllllllllll}
0.3 & 0.5 & 0.7 & 0.9 & 1 & 1.2 & 2 & 3 & 4 & 5 & 6 & 7 & 8 & 9 & 10 & 12
\end{array} \\
& \begin{array}{lllllllllllll}
14 & 15 & 16 & 18 & 20 & 30 & 50 & 75 & 100 & 125 & 150 & 175 & 201
\end{array} \\
& \text { df424 } \log 6.6 \mathrm{E}-12 \quad 9 \mathrm{E}-12 \quad 1.06 \mathrm{E}-11 \quad 1.29 \mathrm{E}-11 \quad 1.35 \mathrm{E}-11 \quad 1.36 \mathrm{E}-11 \\
& \begin{array}{lllll}
1.33 \mathrm{E}-11 & 1.29 \mathrm{E}-11 & 1.2 \mathrm{E}-11 & 1.13 \mathrm{E}-11 & 1.06 \mathrm{E}-11
\end{array} \\
& \begin{array}{llllll}
9.9 \mathrm{E}-12 & 9.4 \mathrm{E}-12 & 8.9 \mathrm{E}-12 & 8.3 \mathrm{E}-12 & 7.9 \mathrm{E}-12 & 7.7 \mathrm{E}-12
\end{array} \\
& 8 \mathrm{E}-12 \quad 1.05 \mathrm{E}-11 \quad 1.66 \mathrm{E}-11 \quad 2.37 \mathrm{E}-11 \quad 4.11 \mathrm{E}-11 \quad 6 \mathrm{E}-11 \\
& \begin{array}{lllll}
8.8 \mathrm{E}-11 & 1.32 \mathrm{E}-10 & 1.7 \mathrm{E}-10 & 2.33 \mathrm{E}-10 & 3.22 \mathrm{E}-10
\end{array} \\
& 3.75 \mathrm{E}-10 \quad 4 \mathrm{E}-10 \quad 4.16 \mathrm{E}-10 \quad 4.25 \mathrm{E}-10 \quad 4.2 \mathrm{E}-10 \quad 4.12 \mathrm{E}-10 \\
& 4.08 \mathrm{E}-10 \quad 4.05 \mathrm{E}-10 \quad 4 \mathrm{E}-10 \quad 4.05 \mathrm{E}-10 \quad 4.09 \mathrm{E}-10 \quad 4.2 \mathrm{E}-10 \\
& \begin{array}{llllll}
4.4 \mathrm{E}-10 & 4.8 \mathrm{E}-10 & 5.2 \mathrm{E}-10 & 5.4 \mathrm{E}-10 & 5.55 \mathrm{E}-10 & 5.7 \mathrm{E}-10
\end{array} \\
& 6 \mathrm{E}-10 \quad 5.15 \mathrm{E}-10 \quad 4 \mathrm{E}-10 \quad 3.3 \mathrm{E}-10 \quad 2.85 \mathrm{E}-10 \quad 2.6 \mathrm{E}-10 \\
& 2.45 \mathrm{E}-10 \quad 2.5 \mathrm{E}-10 \quad 2.6 \mathrm{E}-10
\end{aligned}
$$

The calculated value of tally 414 is equal to $\int_{0}^{\infty}\left(\frac{d \bar{\Phi}_{\gamma}}{d E_{\gamma}}\right)\left(\frac{H_{\gamma}^{*}(10)}{\bar{\Phi}_{\gamma}}\right) d E_{\gamma}$, while the calculated value of tally 424 is equal to $\int_{0}^{\infty}\left(\frac{d \bar{\Phi}_{\mathrm{n}}}{d E_{\mathrm{n}}}\right)\left(\frac{H_{\mathrm{n}}^{*}(10)}{\bar{\Phi}_{\mathrm{n}}}\right) d E_{\mathrm{n}}$. Obtaining the value for $m$ is described in the beginning of this appendix. The calculation then follows Eq. (3.5). 


\section{B Conventional Shielding Calculations}

To calculate photon and neutron dose components inside and outside the bunker door of our bunker design, the method described in NCRP report no. 151 was used ${ }^{7}$.

\section{B.1 Inside Bunker Door}

To calculate the dose inside the door, the dose is broken into different components: primary beam scatter from the room surfaces, head-leakage photon scatter from the room surfaces, primary beam scatter from the patient, head-leakage radiation transmitted through the inner maze wall, neutron capture gamma rays, and neutrons reaching the door. As our maze design is long, and the inner maze wall is thick, it can be shown that neutrons and neutron capture gamma rays are each about two orders of magnitude greater than the other radiation components at the door. Because of this, only the neutron and neutron capture gamma ray components of dose at the door will be shown here. Both of these components rely on the neutron fluence at the inside maze entrance being known, so this value will first be calculated. 
The total neutron fluence at the inside maze entrance per unit absorbed dose from gamma rays at the isocentre, $\varphi_{\mathrm{A}}$, is:

$$
\varphi_{\mathrm{A}}=\frac{\beta Q_{\mathrm{n}}}{4 \pi d_{1}^{2}}+\frac{5.4 \beta Q_{\mathrm{n}}}{2 \pi S_{\mathrm{r}}}+\frac{1.3 Q_{\mathrm{n}}}{2 \pi S_{\mathrm{r}}}
$$

where $\quad \beta \quad$ is the transmission factor for neutrons that penetrate the head shielding

$d_{1} \quad$ is the distance from the isocentre to the inside maze entrance $Q_{\mathrm{n}} \quad$ is the neutron source strength in neutrons emitted from the accelerator head per gray of gamma rays absorbed dose at the isocentre

$S_{\mathrm{r}} \quad$ is the total surface area of the treatment room

The inside maze entrance location is defined as the intersection of the maze's midline and a line that passes through the isocentre and goes as far into the maze as possible without intersecting any walls. The three terms in Eq. (B.1) represent the direct, scattered and thermal neutron components, respectively.

For our bunker design, $\beta$ is assumed to be approximately 0.85 since tungsten is used as head shielding. The distance from the isocentre to the inside maze entrance, $d_{1}$, is measured to be $5.58 \mathrm{~m}$. A value of $8.8 \times 10^{11} \mathrm{~Gy}^{-1}$ is approximated for $Q_{\mathrm{n}}$. Finally, the total surface area of the treatment room, $S_{\mathrm{r}}$, is measured to be $175 \mathrm{~m}^{2}$. Putting these values 
into Eq. (B.1), we obtain:

$$
\begin{aligned}
\varphi_{\mathrm{A}} & =\left(1.91 \times 10^{9}+3.67 \times 10^{9}+1.04 \times 10^{9}\right) \mathrm{m}^{-2} \cdot \mathrm{Gy}^{-1} \\
& =6.63 \times 10^{9} \mathrm{~m}^{-2} \cdot \mathrm{Gy}^{-1}
\end{aligned}
$$

From this, the dose equivalent from neutron capture gamma rays and neutrons can be calculated.

The dose equivalent per MU at the door due to neutron capture gamma rays, $H_{\mathrm{cg}}$, is:

$$
H_{\mathrm{cg}}=W_{\mathrm{L}} K \varphi_{\mathrm{A}} 10^{-\frac{d_{2}}{T V D}}
$$

where $\quad W_{\mathrm{L}} \quad$ is the photon absorbed dose delivered at the isocentre per MU

$K \quad$ is the ratio of the neutron capture gamma ray dose equivalent to the total neutron fluence at the maze entrance

$\varphi_{\mathrm{A}} \quad$ is the total neutron fluence at the maze entrance per unit absorbed dose of gamma rays at the isocentre

$d_{2} \quad$ is the distance from the maze entrance to the door

TVD is the tenth-value distance of neutron capture gamma rays in a maze

At our facility, $W_{\mathrm{L}}$ is set to be $10 \frac{\mathrm{mSv}}{\mathrm{MU}}$. From an average of measurements taken at multiple facilities, $K$ is estimated as $6.9 \times 10^{-16} \mathrm{~Sv} \cdot \mathrm{m}^{2}$. The value of $\varphi_{\mathrm{A}}$ is set to the value 
calculated in Eq. (B.2), $\varphi_{\mathrm{A}}=6.63 \times 10^{9} \mathrm{~m}^{-2} \cdot \mathrm{Gy}^{-1}$. The distance $d_{2}$ is measured to be $6.37 \mathrm{~m}$. Finally, $T V D$ is estimated at $5.4 \mathrm{~m}$ for the $18 \mathrm{MV}$ beam used. Substituting these values into Eq. (B.3), a value of $H_{\mathrm{cg}}=3.02 \times 10^{-6} \frac{\mathrm{mSv}}{\mathrm{MU}}$ is obtained.

The dose equivalent per MU at the door due to neutrons, $H_{\mathrm{n}}$, is:

$$
H_{\mathrm{n}}=\left(2.4 \times 10^{-15} \mathrm{~Sv} \cdot \mathrm{m}^{2}\right) W_{\mathrm{L}} \varphi_{\mathrm{A}} \sqrt{\frac{S_{0}}{S_{1}}}\left(1.64 \times 10^{-\frac{d_{2}}{1.9 \mathrm{~m}}}+10^{-\frac{d_{2}}{2.06 \sqrt{S_{1}}}}\right)
$$

where $W_{\mathrm{L}} \quad$ is the photon absorbed dose delivered at the isocentre per MU

$\varphi_{\mathrm{A}} \quad$ is the total neutron fluence at the maze entrance per unit absorbed dose of gamma rays at the isocentre

$S_{0} \quad$ is the inner maze entrance cross-sectional area

$S_{1} \quad$ is the cross-sectional area along the maze

$d_{2} \quad$ is the distance from the maze entrance to the door

For our bunker design, $W_{\mathrm{L}}, \varphi_{\mathrm{A}}$, and $d_{2}$ are set the same as discussed for the previous calculation of the dose equivalent per MU at the door due to neutron capture gamma rays. A value of $6.50 \mathrm{~m}^{2}$ is measured for $S_{0}$, while a value of $6.70 \mathrm{~m}^{2}$ is measured for $S_{1}$. Putting these values into Eq. (B.4), a value of $H_{\mathrm{n}}=3.37 \times 10^{-6} \frac{\mathrm{mSV}}{\mathrm{MU}}$ is obtained.

The dose equivalents calculated for the room-scattered primary beam, room-scattered photon head leakage, and leakage radiation transmitted through the inner maze wall, together, total only $4.59 \times 10^{-8} \frac{\mathrm{mSv}}{\mathrm{MU}}$. Assembling this quantity, along with the neutron capture gamma ray and neutron components results in Table B.1 . 


\begin{tabular}{|c|c|c|}
\hline \multicolumn{3}{|c|}{ Dose Equivalent per MU $\left(\frac{\mathrm{mSv}}{\mathrm{MU}}\right)$} \\
\hline Photon & Neutron & Total \\
\hline \hline $3.07 \times 10^{-6}$ & $3.37 \times 10^{-6}$ & $6.44 \times 10^{-6}$ \\
\hline
\end{tabular}

TABLE B.1. Photon and neutron dose equivalents per MU calculated for inside the bunker door.

\section{B.2 Outside Bunker Door}

The calculation of dose equivalent outside the bunker door is obtained by taking the dose equivalent inside the bunker door, shown in Table B.1, and modifying it to account for interactions within the door. Specifically, photon and neutron attenuation is considered, along with the production of neutron capture gamma rays. The door of the bunker is made out of 4 layers, from inside the bunker to outside:

1. $0.48 \mathrm{~cm}$ steel

2. $1.27 \mathrm{~cm}$ lead

3. $5.08 \mathrm{~cm} 5 \%$ borated polyethylene

4. $0.48 \mathrm{~cm}$ steel

First, the neutron component is attenuated. The most significant absorption will be in the borated polyethylene. So, the neutron dose equivalent outside the door per $\mathrm{MU}, H_{\mathrm{n}, \text { out }}$, is:

$$
H_{\mathrm{n}, \mathrm{out}}=H_{\mathrm{n}} 10^{-\frac{t_{\mathrm{BPE}}}{T V L_{\mathrm{BPE}, \mathrm{n}}}}
$$


where $H_{\mathrm{n}} \quad$ is the dose equivalent per MU at the door due to neutrons

$t_{\mathrm{BPE}} \quad$ is the thickness of the $5 \%$ borated polyethylene
$T V L_{\mathrm{BPE}, \mathrm{n}} \quad$ is tenth-value layer thickness for neutrons in $5 \%$ borated
polyethylene for the expected neutron spectrum

For our bunker door, $H_{\mathrm{n}}$ is calculated to be $3.37 \times 10^{-6} \frac{\mathrm{mSv}}{\mathrm{MU}}$ from Table B.1, $t_{\mathrm{BPE}}$ is $5.08 \mathrm{~cm}$, and $T V L_{\mathrm{BPE}, \mathrm{n}}$ is estimated to be $3 \mathrm{~cm}$. Putting those numbers into Eq. B.5, a value of $H_{\mathrm{n}, \text { out }}=6.83 \times 10^{-8} \frac{\mathrm{mSv}}{\mathrm{MU}}$ is obtained, meaning that $H_{\mathrm{n}, \text { cap }}=3.30 \times 10^{-6} \frac{\mathrm{mSv}}{\mathrm{MU}}$ is captured.

The photon component is more difficult to calculate. First, the photons are attenuated in the initial steel and lead layers, evaluating the attenuated photon dose equivalent per MU before the borated polyethylene layer, $H_{\mathrm{p}}^{\prime}$ :

$$
H_{\mathrm{p}}^{\prime}=H_{p} 10^{-\frac{t_{\text {steel }}}{T V L_{\text {steel }, \mathrm{p}}}} 10^{-\frac{t_{\text {lead }}}{T V L_{\text {lead }, \mathrm{p}}}}
$$

where $\quad H_{\mathrm{p}} \quad$ is the dose equivalent per MU at the door due to photons

$$
\begin{aligned}
& t_{\text {steel }} \quad \text { is the thickness of the steel } \\
& T V L_{\text {steel,p }} \quad \text { is tenth-value layer thickness for photons in steel for the ex- } \\
& \text { pected photon spectrum } \\
& \text { is the thickness of the lead } \\
& t_{\text {lead }} \quad \text { is tenth-value layer thickness for photons in lead for the } \\
& T V L_{\text {lead,p }} \\
& \text { expected photon spectrum }
\end{aligned}
$$


For our bunker door, $H_{\mathrm{p}}$ is calculated to be $3.07 \times 10^{-6} \frac{\mathrm{mSv}}{\mathrm{MU}}$ from Table B.1. Thicknesses of $0.48 \mathrm{~cm}$ for $t_{\text {steel }}$ and $1.27 \mathrm{~cm}$ for $t_{\text {lead }}$ are obtained from the door specifications, and estimates of $10 \mathrm{~cm}$ for $T V L_{\mathrm{steel}, \mathrm{p}}$ and $5.7 \mathrm{~cm}$ for $T V L_{\mathrm{lead}, \mathrm{p}}$ were used. Putting those numbers into Eq. B.6, a value of $H_{\mathrm{p}}^{\prime}=1.65 \times 10^{-6} \frac{\mathrm{mSv}}{\mathrm{MU}}$ is obtained.

The neutron capture gamma rays created in the borated polyethylene can be estimated by the "intuitive" method discussed by $\mathrm{McCall}^{57}$. The neutron capture gamma rays dose equivalent per MU created in the borated polyethylene, $H_{\mathrm{cg}}^{\prime}$ is:

$$
H_{\mathrm{cg}}^{\prime}=k_{\mathrm{BPE}} H_{\mathrm{n}, \mathrm{cap}}
$$

where $k_{\mathrm{BPE}} \quad$ is the conversion coefficient from captured neutron dose equivalent to photon dose equivalent in borated polyethylene

$H_{\mathrm{n}, \mathrm{cap}} \quad$ is the dose equivalent per MU of neutrons captured in the borated polyethylene

Using a value of 0.1115 for $k_{\mathrm{BPE}}$, and the calculated value of $H_{\mathrm{n}, \mathrm{cap}}=3.30 \times 10^{-6} \frac{\mathrm{mSv}}{\mathrm{MU}}$, a result of $H_{\mathrm{cg}}^{\prime}=3.68 \times 10^{-7} \frac{\mathrm{mSv}}{\mathrm{MU}}$ is obtained.

The attenuated photon component, $H_{\mathrm{p}}^{\prime}$, and the neutron capture gamma ray component, $H_{\mathrm{cg}}^{\prime}$, can then be combined, and attenuated throughout the rest of the door layers. The final result is the photon dose equivalent outside the door per MU, $H_{\mathrm{p}, \text { out }}$ :

$$
H_{\mathrm{p}, \mathrm{out}}=\left(H_{\mathrm{p}}^{\prime}+H_{\mathrm{cg}}^{\prime}\right) 10^{-\frac{t_{\mathrm{BPE}}}{T V L_{\mathrm{BPE}, \mathrm{p}}}} 10^{-\frac{t_{\text {steel }}}{T V L_{\text {steel }, \mathrm{p}}}}
$$




\begin{tabular}{|c|c|c|}
\hline \multicolumn{3}{|c|}{ Dose Equivalent per MU $\left(\frac{\mathrm{mSv}}{\mathrm{MU}}\right)$} \\
\hline Photon & Neutron & Total \\
\hline \hline $1.483 \times 10^{-6}$ & $6.83 \times 10^{-8}$ & $1.552 \times 10^{-6}$ \\
\hline
\end{tabular}

TABLE B.2. Photon and neutron dose equivalents per MU calculated for outside the bunker door.

where $\quad H_{\mathrm{p}}^{\prime} \quad$ is the attenuated photon dose equivalent per MU before the borated polyethylene layer

$H_{\mathrm{cg}}^{\prime} \quad$ is the neutron capture gamma rays dose equivalent per $\mathrm{MU}$ created in the borated polyethylene

$t_{\mathrm{BPE}} \quad$ is the thickness of the borated polyethylene

$T V L_{\mathrm{BPE}, \mathrm{p}} \quad$ is tenth-value layer thickness for photons in borated polyethylene for the expected photon spectrum

$t_{\text {steel }} \quad$ is the thickness of the steel

$T V L_{\text {steel,p }}$ is tenth-value layer thickness for photons in steel for the expected photon spectrum

To evaluate the final value for the photon dose equivalent outside the door per MU, values calculated above of $H_{\mathrm{p}}^{\prime}=1.65 \times 10^{-6} \frac{\mathrm{mSv}}{\mathrm{MU}}$ and $H_{\mathrm{cg}}^{\prime}=3.68 \times 10^{-7} \frac{\mathrm{mSv}}{\mathrm{MU}}$ are used. Thicknesses of $5.08 \mathrm{~cm}$ for $t_{\mathrm{BPE}}$ and $0.48 \mathrm{~cm}$ for $t_{\mathrm{steel}}$ are obtained from the door specifications, and values of $60 \mathrm{~cm}$ for $T V L_{\mathrm{BPE}, \mathrm{p}}$ and $10 \mathrm{~cm}$ for $T V L_{\mathrm{steel}, \mathrm{p}}$ are also used. Putting those numbers into Eq. B.8, a value of $H_{\mathrm{p}, \text { out }}=1.48 \times 10^{-6} \frac{\mathrm{mSv}}{\mathrm{MU}}$ is obtained.

Putting together the photon and neutron components for outside the door, Table B.2 is obtained. 


\section{References}

${ }^{1}$ T. Brecht, "A simple approach to thesis writing," (2006), URL http://bcr2. uwaterloo.ca/ brecht/thesis-hints.html.

${ }^{2}$ International Commission on Radiological Protection, "Conversion coefficients for use in radiological protection against external radiation-ICRP publication 74," Ann. ICRP 26(3-4), 1-205 (1996).

${ }^{3}$ C. Wernli, "External dosimetry: Operational quantities and their measurement," (2006), URL http://w3.tue.nl/fileadmin/sbd/Documenten/IRPA_refresher_courses/ External_Dosimetry_operational_Quantities_and_their_Measurement.pdf.

${ }^{4}$ International Atomic Energy Agency, "IAEA safety glossary-terminology used in nuclear, radiation, radioactive waste and transport safety 1.0," Online (2000).

${ }^{5}$ F. H. Attix, Introduction to Radiological Physics and Radiation Dosimetry (WileyInterscience, New York, 1986).

${ }^{6} \mathrm{X}-5$ Monte Carlo Team, MCNP-A general Monte Carlo N-Particle transport code, version 5, Los Alamos National Laboratory, Los Alamos, New Mexico (2003). 
${ }^{7}$ National Council on Radiation Protection and Measurements, Structural Shielding Design And Evaluation for Megavoltage X-and Gamma-ray Radiotherapy FacilitiesNCRP Report 151 (Natl Council on Radiation, 2005).

${ }^{8}$ A. Ahnesjo and M. M. Aspradakis, "Dose calculations for external photon beams in radiotherapy," Phys. Med. Biol. (UK) 44(11), 99-155 (1999).

${ }^{9}$ E. W. Weisstein, "Monte Carlo method," From MathWorld-A Wolfram Web Resource (2006), URL http://mathworld.wolfram.com/MonteCarloMethod.html.

${ }^{10}$ A. F. Bielajew, Fundamentals of the Monte Carlo method for neutral and charged particle transport (University of Michigan, Ann Arbor, Michigan, 2001), URL http: //www-personal.engin.umich.edu/ bielajew/MCBook/book.pdf.

${ }^{11}$ E. W. Weisstein, “Torus," From MathWorld-A Wolfram Web Resource (2006), URL http://mathworld.wolfram.com/Torus.html.

${ }^{12}$ E. J. Hall, Radiobiology for the Radiologist (Lippincott Williams \& Wilkins, Philadelphia, 2005), 6th ed.

${ }^{13}$ E. B. Podgorsak, editor, Radiation Oncology Physics: A Handbook for Teachers and Students (International Atomic Energy Agency, 2005).

${ }^{14}$ P. H. McGinley, Shielding Techniques for Radiation Oncology Facilities (Medical Physics Publishing Corporation, 2002), 2nd ed.

${ }^{15}$ Canadian Nuclear Safety Commission, Keeping Radiation Exposures and Doses “As Low as Reasonably Achievable (ALARA)”, G-129, Revision 1 (Canadian Nuclear Safety Com- 
mission, Ottawa, Ontario, 2004), URL http://www.nuclearsafety.gc.ca/pubs_ catalogue/uploads/G129revl_e.pdf.

${ }^{16} \mathrm{~F}$. Verhaegen and J. Seuntjens, "Monte Carlo modelling of external radiotherapy photon beams," Phys. Med. Biol. (UK) 48(21), 107-164 (2003).

${ }^{17}$ R. Jeraj, P. J. Keall, and P. M. Ostwald, "Comparisons between MCNP, EGS4 and experiment for clinical electron beams," Phys. Med. Biol. (UK) 44(3), 705-717 (1999).

${ }^{18}$ MCNP Team, "Los Alamos National Laboratory: MCNP home page," (2006), URL http://mcnp-green.lanl.gov/.

${ }^{19}$ Stanford Linear Accelerator Center, "Electron gamma shower (EGS) Monte Carlo radiation transport code," (2006), URL http://www.slac.stanford.edu/egs/.

${ }^{20}$ D. W. O. Rogers, B. A. Faddegon, G. X. Ding, C.-M. Ma, J. We, and T. R. Mackie, "BEAM: a Monte Carlo code to simulate radiotherapy treatment units," Med. Phys. (USA) 22(5), 503-24 (1995).

${ }^{21}$ E. L. Chaney, T. J. Cullip, and T. A. Gabriel, "A Monte Carlo study of accelerator head scatter," Med. Phys. (USA) 21(9), 1383-1390 (1994).

${ }^{22}$ R. D. Lewis, S. J. S. Ryde, D. A. Hancock, and C. J. Evans, "An MCNP-based model of a linear accelerator X-ray beam," Phys. Med. Biol. (UK) 44(5), 1219-1230 (1999).

${ }^{23}$ J. V. Siebers, P. J. Keall, B. Libby, and R. Mohan, "Comparison of EGS4 and MCNP4b Monte Carlo codes for generation of photon phase space distributions for a Varian 2100C," Phys. Med. Biol. (UK) 44(12), 3009-3026 (1999). 
${ }^{24}$ D. Sheikh-Bagheri, D. W. O. Rogers, C. K. Ross, and J. P. Seuntjens, "Comparison of measured and Monte Carlo calculated dose distributions from the NRC linac," Med. Phys. (USA) 27(10), 2256-2266 (2000).

${ }^{25}$ S.-Y. Lin, T.-C. Chu, and J.-P. Lin, "Monte Carlo simulation of a clinical linear accelerator," Appl. Radiat. Isot. (UK) 55(6), 759-765 (2001).

${ }^{26}$ D. Sheikh-Bagheri and D. W. O. Rogers, "Sensitivity of megavoltage photon beam Monte Carlo simulations to electron beam and other parameters," Med. Phys. (USA) 29(3), 379390 (2002).

${ }^{27}$ D. Sheikh-Bagheri and D. W. O. Rogers, "Monte Carlo calculation of nine megavoltage photon beam spectra using the BEAM code," Med. Phys. (USA) 29(3), 391-402 (2002).

${ }^{28}$ E. C. Schreiber and B. A. Faddegon, "Sensitivity of large-field electron beams to variations in a Monte Carlo accelerator model," Phys. Med. Biol. (UK) 50(5), 769-778 (2005).

${ }^{29}$ B. Serrano, A. Hachem, E. Franchisseur, J. Hérault, S. Marcié, A. Costa, R. J. Bensadoun, J. Barthe, and J. P. Gérard, "Monte Carlo simulation of a medical linear accelerator for radiotherapy use," Radiat. Prot. Dosim. (UK) (2006), URL http://rpd. oxfordjournals.org/cgi/reprint/nci620v1. Electronic publication to be printed.

${ }^{30}$ C. Ongaro, J. Ródenas, A. Leon, J. Perez, A. Zanini, and K. Burn, "Monte Carlo simulation and experimental evaluation of photoneutron spectra produced in medical linear accelerators," "Proceedings of the 1999 Particle Accelerator Conference," (New York, NY, USA, 1999), vol. 4, 2531-2533. 
${ }^{31}$ C. Ongaro, A. Zanini, U. Nastasi, J. Ródenas, G. Ottaviano, and C. Manfredotti, “Analysis of photoneutron spectra produced in medical accelerators," Phys. Med. Biol. (UK) 45(12), L55-L61 (2000).

${ }^{32}$ O. Chibani and C.-M. C. Ma, "Photonuclear dose calculations for high-energy photon beams from Siemens and Varian linacs," Med. Phys. (USA) 30(8), 1990-2000 (2003).

${ }^{33}$ D. S. Followill, M. S. Stovall, S. F. Kry, and G. S. Ibbott, "Neutron source strength measurements for Varian, Siemens, Elekta, and general electric linear accelerators," J. Appl. Clin. Med. Phys. (USA) 4(3), 189-194 (2003).

${ }^{34}$ R. Barquero, R. Mendez, H. R. Vega-Carrillo, M. P. Iñiguez, and T. M. Edwards, "Neutron spectra and dosimetric features around an $18 \mathrm{MV}$ linac accelerator," Health Phys. (USA) 88(1), 48-58 (2005).

${ }^{35}$ J. Pena, L. Franco, F. Gomez, A. Iglesias, J. Pardo, and M. Pombar, "Monte Carlo study of Siemens Primus photoneutron production," Phys. Med. Biol. (UK) 50(24), 5921-5933 (2005).

${ }^{36}$ J. J. DeMarco, T. D. Solberg, R. E. Wallace, and J. B. Smathers, "A verification of the Monte Carlo code MCNP for thick target bremsstrahlung calculations," Med. Phys. (USA) 22(1), 11-16 (1995).

${ }^{37}$ E. Carinou, V. Kamenopoulou, and I. E. Stamatelatos, "Evaluation of neutron dose in the maze of medical electron accelerators," Med. Phys. (USA) 26(12), 2520-2525 (1999).

${ }^{38}$ I. A. M. Al-Affan, "Estimation of the dose at the maze entrance for X-rays from radiotherapy linear accelerators," Med. Phys. (USA) 27(1), 231-238 (2000). 
${ }^{39}$ National Council on Radiation Protection and Measurements, Radiation Protection Design Guidelines for 0.1-100 MeV Particle Accelerator Facilities-NCRP Report 51 (Natl Council on Radiation, 1977).

${ }^{40}$ National Council on Radiation Protection and Measurements, Radiation Protection for Particle Accelerator Facilities-NCRP Report 144 (Natl Council on Radiation, 2003).

${ }^{41}$ P. H. McGinley, A. H. Dhaba'an, and C. S. Reft, "Evaluation of the contribution of capture gamma rays, $\mathrm{X}$-ray leakage, and scatter to the photon dose at the maze door for a high energy medical electron accelerator using a Monte Carlo particle transport code," Med. Phys. (USA) 27(1), 225-230 (2000).

${ }^{42}$ P. H. McGinley, M. S. Miner, and M. L. Mitchum, "A method for calculating the dose due to capture gamma rays in accelerator mazes," Phys. Med. Biol. (UK) 40(9), 1467-73 (1995).

${ }^{43}$ J.-P. Lin, T.-C. Chu, S.-Y. Lin, and M.-T. Liu, "The measurement of photoneutrons in the vicinity of a Siemens Primus linear accelerator," Appl. Radiat. Isot. (UK) 55(3), 315-321 (2001).

${ }^{44}$ M. A. Avila-Rodriguez, P. M. DeLuca, Jr, and T. D. Bohm, "Simulation of medical electron linac bremsstrahlung beam transport in typical shielding materials," Radiat. Prot. Dosim. (UK) 116(1-4), 547-552 (2005).

${ }^{45}$ R. A. Forster, L. J. Cox, R. F. Barrett, T. E. Booth, J. F. Briesmeister, F. B. Brown, J. S. Bull, G. C. Geisler, J. T. Goorley, R. D. Mosteller, S. E. Post, R. E. Prael, E. C. Selcow, and A. Sood, "MCNP ${ }^{\mathrm{TM}}$ version 5," Nucl. Instrum. Methods Phys. Res. B, Beam Interact. Mater. At. (Netherlands) 213, 82-86 (2004). 
${ }^{46}$ International Atomic Energy Agency, "IAEA photonuclear data," (2006), URL http: //t2.lanl.gov/data/photonuclear.html.

${ }^{47}$ M. J. Berger, J. S. Coursey, M. A. Zucker, and J. Chang, “ESTAR, PSTAR, and ASTAR: Computer programs for calculating stopping-power and range tables for electrons, protons, and helium ions (version 1.2.3)," National Institute of Standards and Technology (2006), URL http://physics.nist.gov/Star. Originally published as: Berger, M.J., NISTIR 4999, National Institute of Standards and Technology, Gaithersburg, MD (1993).

${ }^{48}$ D. R. Schaart, J. T. M. Jansen, J. Zoetelief, and P. F. A. de Leege, "A comparison of MCNP4C electron transport with ITS 3.0 and experiment at incident energies between $100 \mathrm{keV}$ and $20 \mathrm{MeV}$ : influence of voxel size, substeps and energy indexing algorithm," Phys. Med. Biol. (UK) 47(9), 1459-1484 (2002).

${ }^{49}$ M. A. F. Lima, A. X. Silva, and V. R. Crispim, "Calculation of conversion coefficients for clinical photon spectra using the MCNP code," Radiat. Prot. Dosim. (UK) 111(1), 9-12 (2004).

${ }^{50}$ K. G. Veinot and N. E. Hertel, "Effective quality factors for neutrons based on the revised ICRP/ICRU recommendations," Radiat. Prot. Dosim. (UK) 115(1-4), 536-541 (2005).

${ }^{51}$ International Commission on Radiation Units and Measurements, Quantities and Units in Radiation Protection Dosimetry-ICRU Report 51 (International Commission on Radiation Units and Measurements, Bethesda, MD, 1993).

${ }^{52}$ T. E. Booth, “A sample problem for variance reduction in MCNP,” Tech. Rep. LA-10363MS, Los Alamos National Laboratory, Los Alamos, New Mexico (1985). 
${ }^{53}$ National Research Council of Canada, "Beamnrc home page," (2006), URL http: // www.irs.inms.nrc.ca/BEAM/beamhome.html.

${ }^{54}$ Thermo Electron Corporation, "Neutron shielding material catalog no. 201," (2006), URL http://www.thermo.com/eThermo/CMA/PDFs/Product/productPDF_24114. pdf.

${ }^{55}$ MCNP Team, "MCNP 5.1.40 RSICC release notes," (2006), URL http:// monp-green.lanl.gov/pdf/LA_UR_05-86171.pdf.

${ }^{56}$ B. R. L. Siebert and H. Schuhmacher, “Quality factors, ambient and personal dose equivalent for neutrons, based on the new ICRU stopping power data for protons and alpha particles," Radiat. Prot. Dosim. (UK) 58(3), 177-183 (1995).

${ }^{57}$ R. C. McCall, "Shielding for thermal neutrons," Med. Phys. (USA) 24(1), 135-136 (1997). 